GROUND-WATER PUMPAGE IN NASSAU COUNTY,

LONG ISLAND, NEW YORK 1920-77

INTRODUCTION AND USER'S GUIDE TO THE DATA COMPILATION

By Chabot Kilburn

U.S. GEOLOGICAL SURVEY

Open-File Report 81-499

Prepared in cooperation with

NASSAU COUNTY DEPARTMENT OF PUBLIC WORKS

Syosset, New York

1982 
UNITED STATES DEPARTMENT OF THE INTERIOR

JAMES G. WATT, Secretary

GEOLOGICAL SURVEY

Dallas L. Peck, Director

For additional information write to:

U.S. Geological Survey

5 Aerial Way

Syosset, New York 11791 


\section{PLATES}

(in pocket)

Plates 1 and 2. Maps of Nassau County showing:

1. Location of public water-supply wells from 1920 to 1977 and boundaries of water-supply areas

2. Location of nonpublic water-supply wells that are required to report ground-water pumpage and other wells for which ground-water pumpage is available

\section{TABLES}

Explanation of columns and abbreviations in Tables 1 and 2 .

Table 1. We11-completion data for public water-supply wells in

Nassau County from 1920 to 1977 . . . . . . . . .

2. We11-completion data on nonpublic water-supply wells in Nassau County that are required to report ground-water pumpage and other wells for which ground-water pumpage is available... . . . . . . . . . . . . . 


\title{
GROUND-WATER PUMPAGE IN NASSAU COUNTY, LONG ISLAND, NEW YORK, 1920-77 \\ Introduction and User's Guide to the Data Compilation
}

by

Chabot Kilburn

\begin{abstract}
Pumpage data from approximately 2,100 wells used for public and nonpublic water supply in Nassau County from 1920-77 have been collected and are available for public inspection at the U.S. Geological Survey office in Syosset, Nassau County. Except for wells in use before 1920, these data constitute the entire available record through 1977. The data are divided into two categories-pumpage from wells used for public supply and pumpage from wells used mainly for industrial and commercial supply, designated as nonpublic water supply. The pumpage is tabulated by well or well field and is given by month or year, with an annual total.

This report serves as an introduction and user's manual to the data, which are available for inspection but not included herein because they fill several hundred computer pages. This report describes the method of reporting pumpage on Long Island and gives information on how pumpage values were derived from the records. It also includes we11-completion data and two maps showing the location of all wells.
\end{abstract}




\section{INTRODUCTION}

The increasing use of ground water in Nassau County has brought about the need for detailed hydrologic data to aid in the study of the ground-water system underlying the county. The data are needed to aid in (1) constructing analog and computer models of the hydrologic system used to predict effects of anticipated stresses on the system; (2) water-quality and wastewater-disposal studies; (3) studies of long-term changes in ground-water levels, withdrawals, and chemical quality; and (4) studies of artificial recharge. This report is a guide to a new, comprehensive compilation of data on ground-water withdrawals in Nassau County.

The data are not included in this volume owing to their large bulk but are available for inspection in computer-printout format at the Long Island, N.Y., office of the U.S. Geological Survey.

\section{Purpose and Scope}

The purpose of this report is to describe the new compilation of Nassau County data on ground-water pumpage collected or estimated by government agencies from 1920 to 1977. The data consist of all pumpage records from Nassau County collected by or filed at the New York State Department of Environmental Conservation at Stony Brook (hereafter referred to as NYSDEC), the Nassau County Department of Public Works, Division of Sanitation and Water Supply in Bellmore, and the U.S. Geological Survey in Syosset. All data have been tabulated on a monthly and annual basis and are listed by well number or well field. They constitute the period of record from 1920 through 1977. The data are divided into two categories--pumpage from approximately 1,700 wells used for public supply, and pumpage from approximately 400 wells used mainly for industrial and commercial supply, referred to herein as nonpublic supply. The term public-supply pumpage in this report refers only to water pumped from wells owned by public water-supply agencies regardless of its ultimate customer or use.

\section{SOURCES OF WELL AND WELL-FIELD DATA}

Information on past and present wells and well fields in Nassau County was obtained primarily from water-supply applications (which relate to public water supplies) and Long Island well applications (which relate to nonpublic water supplies), which are filed with the NYSDEC in Stony Brook, Long Island. Additional information on some public water-supply wells was obtained from the the U.S. Geological Survey office in Syosset, Nassau County. The locations of public water-supply wells and well fields in existence during 1920-77 are shown in plate 1; locations of nonpublic water supply wells that are required to report pumpage and other wells whose pumpage is reported voluntarily are shown in plate 2. Note, however, that pumpage information on some nonpublic supply wells could not be found and is assumed to be unavailable.

Well-completion data, including well depths and aquifers from which the wells obtain water, are listed in table 1 for public water-supply wells and in table 2 for nonpublic water-supply wells. 


\title{
REPORTING OF PUMPAGE
}

\section{New York State Requirements}

In 1933, the New York State Legislature, as part of the Conservation Law, required that anyone planning to install a well for any purpose other than agriculture on Long Island must obtain the consent of the New York State Water Power and Control Commission (now the New York State Department of Environmental Conservation), if the capacity of the we11, or the total capacity of any old or new wells together on one property, would exceed 100,000 gallons per day ( 69.44 gallons per minute).

To receive permission to install and operate such a well, an application must be made to NYSDEC for consideration and approval. In giving such approva1, NYSDEC has authority to impose conditions and make such requirements and limitations as deemed necessary. One condition frequently imposed is that all water pumped from a we 11 be metered or its volume estimated and that the meter readings or estimates be recorded and retained by the we11 owner and reported month1y or annually to NYSDEC.

On July 1, 1954, NYSDEC reduced the reporting requirement for any wel1 on Long Island to 45 gallons per minute.

\section{Methods of Determining and Reporting Pumpage}

\author{
Public Water Supplies
}

Al1 public water-supply we1ls in Nassau County are metered. The water-supply operators tabulate pumpage for each well monthly and annually on forms prepared by the Nassau County Department of Public Works. At the end of each year they send the forms to the County's Division of Public Works in Bellmore. If meters break or malfunction during the year, the pumpage is generally reported as an estimate.

\section{Nonpublic Water Supplies}

The conditions imposed by NYSDEC in decisions relating to nonpublic water supplies generally state:

Applicant shall install, maintain and operate a satisfactory meter or other device to measure and record the amount of water pumped from the proposed well and shall preserve such records... Applicant shall report the amount of pumpage monthly to this Department.

In some instances, the pumpage is required to be reported annually rather than monthly.

Nearly all nonpublic supply wells that are required to report pumpage, or that report pumpage voluntarily, are equipped with cumulating meters. A few, however, are equipped with hour meters that indicate the cumulative hours of operation of the pump. Pumpage from these wells is calculated by multiplying 
the hours of operation by the reported or rated yield of the well. For some wells of low yield and small usage, NYSDEC may request only that the pumpage be estimated and reported annually.

NYSDEC requests that nonpublic water-supply pumpage from wells equipped either with cumulating meters or hour meters be reported in the following manner :

\author{
We11 number \\ Date of last meter reading \\ Meter reading \\ Date of previous meter reading \\ Meter reading \\ Total consumption in gallons
}

However, many well operators fail to follow this format when reporting pumpage, and some fail to report pumpage on a monthly basis. Many tabulate pumpage for varying periods of time rather than by month, and some report only at irregular intervals throughout the year, or in some years not at all. Some operators do not properly designate the pumped well or wells, and many omit the numbers assigned to the wells by NYSDEC. Therefore, the available record is not always complete.

\title{
SOURCES OF PUMPAGE DATA
}

\section{Public-Supply Pumpage}

\author{
1920 to 1933
}

A11 public water-supply pumpage data for 1920-33 in Nassau County were obtained from files of the U.S. Geological Survey in Syosset. These data were probably part of those used by Thompson and Leggette (1936) in determining the withdrawal of ground water on Long Island from 1904 to 1934. Thompson and Leggette (1936, p. 7) state:

The records of withdrawals [for this period] ...were obtained either from the files of the water-supply organizations or from published reports. For some of the smaller water supplies in Nassau County, where no such records were available, it was necessary to estimate the withdrawals.

\section{4 to 1936}

Pumpage data for many wells in Nassau County from 1934-36 are unavailable. Many of the data were obtained from the files of the U.S. Geological Survey and consist of hand-copied records of uncertain origin. These records are assumed to have been obtained from water-supply owners. 
Since 1937, all ground-water pumpage for public supply in Nassau County has been reported to the Nassau County Department of Public Works. These reports are prepared by the individual public water-supply agencies, which, with some exceptions, report the pumpage annually by well or well field. Exceptions are when the combined pumpage of two or more wells is reported as a single combined sum or was measured by a single meter. The latter type also includes pumpage from suction-well systems, which consist of a group of many wells that are pumped by a central suction-type pump.

The public water-supply pumpage data given in this report were obtained from the reports on ground-water pumpage in the files of the Nassau County Department of Public Works, Division of Sanitation and Water Supply, in Bellmore.

\title{
Nonpublic-Supply Pumpage
}

\author{
1935 to 1945
}

The first nonpublic water-supply we11 in Nassau County to be issued an operating permit by the Water Power and Control Commission (now NYSDEC) was we11 N-102, which was operated by the Wheatley Hills Golf Club. The permit was issued in the latter part of 1935 and required that the well be metered and that the meter readings be recorded and preserved by the club. It was not until 1943, however, that pumpage was required to be reported to the Commission; this was stipulated to be on a monthly basis. The first well affected by this latter requirement was $\mathrm{N}-1788$, which belonged at that time to the Strathmore Realty Company. The earliest records of pumpage for well $\mathrm{N}-1788$ and other wells in Nassau County begin in 1946; these are stored in the files of the Water Supply Unit of NYSDEC. Data for nonpublic water-supply pumpage, 1935 through 1945, were not found but may be filed by the water-supply owners.

\section{6 to 1968}

Data on ground-water pumpage for 1946-68 were obtained from the files of the Water Supply Unit of NYSDEC in Stony Brook. The data consist almost entirely of typed summaries compiled by the Water Supply Unit from pumpage reports sent to them by the individual companies or well users. If the reported pumpage was from two or more wells belonging to a single owner or user, it was generally reported as a total pumpage from all we11s. The pumpage was given by month and year, with an annual total. Also available were (1) industrial pumpage summaries from 1950-56, from which the total pumpage by owner could be calculated, and (2) some original pumpage reports sent to the Water Supply Unit by the well users. All data from this latter group that were available were used in this report in preference to the Water Supply Unit's pumpage summaries.

\section{9 to 1977}

The data used for tabulating the nonpublic water-supply pumpage in Nassau County from 1969-77 were obtained from the water-supply users' original pumpage reports from files of the Water Supply Unit. Occasionally, when some of the 
original reports for this period were missing, the needed data were taken from the annual summaries. (Some time before this study, most of the original pumpage reports for the years preceding 1969 were discarded because they were thought to be no longer needed by the Water Supply Unit.)

Some data on nonpublic water-supply pumpage in the files of the Water Supply Unit are from sources that were not required to report pumpage. These data have been reported voluntarily to NYSDEC to aid NYSDEC in acquiring information on ground-water use that may be helpful in making future decisions relating to ground-water management. These data are also included in the computer compilations; the location of these wells is shown in plate 2.

\section{FORMAT OF PUMPAGE-DATA COMPILATION}

In the computer compilations at the Geological Survey's Long Island office, pumpage is given by well or well field. Yearly pumpage is given by month, with an annual total. Pumpage given is for the period of available record for wells in use since 1920. For convenience, the records have been divided into two parts: part 1 contains public water-supply pumpage and is given in four sections; part 2 contains nonpublic water-supply pumpage (industrial, commercial, and other private uses) and is given in two sections. Some pumpage data on wells before 1920 are available at the U.S. Geological Survey office in Syosset.

If the reported pumpage was a sum of pumpage from two or more wells in the same well field and from the same aquifer, it was not represented by well in the computer compilation and is given as reported. However, each of the wells in use, or possibly in use, in the year for which pumpage is reported is identified.

If the reported pumpage is a sum of pumpage from wells at different locations but in the same aquifer, pumpage is broken down by well by either of the following methods: (1) by ratio of average yields for years before or after that year; or (2) by ratio of reported yields of the pumps or as determined during test pumping. If the wells tapped two or more aquifers, total pumpage from each aquifer was first determined from the ratio of the number of wells in each aquifer; then pumpage from each well was determined by one of the methods given above.

If the reported pumpage was from a large well field or from a suction-well system, and if all wells are or were in the same aquifer, pumpage is reported as the well-field total. If the wells tapped two or more aquifers and only the total well-field pumpage was reported, pumpage is broken down by the ratio of wells in each aquifer. Each of the wells in use or possible use in the year for which the pumpage is reported is identified.

\section{REFERENCE CITED}

Thompson, D. D., and Leggette, R. M., 1936, Withdrawal of ground water on Long Island, New York: New York State Water Power and Control Commission Bulletin GW-1, 28 p., 6 figs. 


\section{We11 Number}

We11 numbers are assigned by the New York State Department of Environmental Conservation. The prefix $\mathrm{N}$ designates Nassau County.

\section{Owner or Well User}

The owner or well user is generally the name shown on the completion report that was sent to the New York State Department of Environmental Conservation by the driller. During this study, it was found that many of the wells have changed ownership or user. The last known owner or well user is listed if known.

The following abbreviations are used in the owner or well user column of table 1:

$\mathrm{CO}$

CORP

FRANKLIN SQ. WD

GARD. CTY PK WD

L.I. WATER CORP

LIDO-PT. LKT. WD

LOCUST VLY WD

MANH. -LAKE. WD

MILL NECK ESTS.

N.Y.WTR.SERVICE

NASSAU CO. WTR.

PORT WASH. WD

QUEENS CO WTR

ROCKVILLE CTR

ROOSVLT.FLD.WD

SEL-VRA ACRES

SO. FARMING. WD

WD

WTR

W. HEMP-H. GDS. WD
Company or County

Corporation

Franklin Square Water District

Garden City Park Water District

Long Island Water Corp.

Lido-Point Lookout Water District

Locust Valley Water District

Manhasset-Lakeville Water District

Association of Owners of Mill Neck Estates

New York Water Service Corp.

Nassau County Water Co.

Port Washington Water District

Queens County Water Co.

Rockville Centre

Roosevelt Field Water District

Association of Property Owners of SEL-VRA

Acres

South Farmingdale Water District

Water District

Water

West Hempstead-Hempstead Gardens Water

District 


\section{Owner or Well User (continued)}

The following abbreviations are used in the owner or well user column of table 2:

395 ATL. AV. CORP

AM. PHYSICS INST ASSOC.

AUTOMATIC IND

BELL-MER CHSD 非

BETHPAGE ST. PK.

CC

CL

$\mathrm{CO}$

CORP

CERT. REDI-MIX

CLAREMONT POLY. COLONIAL SAND

CON LITHO CORP

COUNTY COM. CORP

C.W. POST COLL.

EST or ESTS

GC

GENERAL INST.

GLEN COVE BOT.

H. RUB INSTEIN

HOLY ROOD CTRY

HOSP

INC

IND

ISL. TREES ET AL

JONES BEACH PK

KOLLSMAN INST.

LAB. FURNITURE

LEASE PLAN INT.

L. I. HOME LTD

L.I. LIGHTING CO

L.I. RAILROAD CO
395 Atlantic Avenue Corp.

American Institute of Physics

Associates

Automatic Industries, Inc.

Bellmore-Merrick Central High School District

No. 3

Bethpage State Park

County Club

Club

Company

Corporation

Certified Redi-Mix Co., Inc.

Claremont Polychemical Corp.

Colonial Sand \& Stone Co., Inc.

Consolidated Lithographing Corp.

County Community Corp.

C. W. Post College

Estate or estates

Colf club

Genera1 Instrument Corp.

Glen Cove Bottling Co., Inc.

Helena Rubinstein Inc.

Cemetery of the Holy Rood

Hospital

Incorporated

Industries

Island Trees Corp. and others

Jones Beach State Park

Kollsman Instrument Corp.

Laboratory Furniture Co., Inc.

Lease Plan International Corp.

Long Island Home Ltd.

Long Island Lighting Co.

Long Island Railroad Co. 


\section{Owner or Well User (continued)}

M. MAGENHE IMER

MANH. STEAM LDY

MANOR OLDS. MERCHANT MAR AC

N.Y. STATE UNIV. NASSAU CO. MED. NATIONAL PAR 3

NO. SHORE TOWERS

NORTH HEMP. CC

OLD WEST. GARD.

PK

PS

PENN MACHINERY

RIVERSIDE PLAS.

ROOSVLT. FLD. WD

$\mathrm{SCH}$

SO. ST. PKWY. GC

ST. PIUS X SEMIN

ST. UNIV. AT O.W. SPERRY GYRO. CO.

SUNRISE FED SVG

V.S. CTRL. H.S.D.

WHEATLEY HLS GC
METRO. S AND G

Mason $\mathrm{Au} \&$ Magenheimer Confectionery Mfg. Co. Inc.

Manhasset Steam Laundry

Manor 0ldsmobile Inc.

U.S. Merchant Marine Academy

Metropolitan Sand and Gravel Co.

N.Y. State University

Nassau County Medical Center

National Par 3 Golf, Inc.

North Shore Towers

North Hempstead Country Club

O1d Westbury Gardens Inc.

Park

Public schools

H. O. Penn Machinery Co.

Riverside Plastics Division, Bischoff Chemical Corp.

Roosevelt Field Water District

School

Southern State Parkway Golf Club

St. Pius X Seminary of the Diocese of

Rockville Centre

State University of New York at Old Westbury

Sperry Gyroscope Division of Sperry Rand

Corp.

Sunrise Federal Saving and Loan Association

Valley Stream Central High School District

Wheatley Hills Golf Club, Inc.

\section{Year Completed}

Year completed refers to the year in which the well was reported to have been completed or accepted by the original well owner. It may not always be the year in which the well was drilled.

Altitude of LSD (Land-Surface Datum)

(feet above National Geodetic Vertical Datum of 1929)

The altitude of land surface at the well was estimated from U.S. Geological Survey 7-1/2-minute quadrangle topographic maps. 
EXPLANATION OF COLUMNS AND ABBREVIATIONS IN TABLES 1 AND 2 (continued)

Use of Water

The following abbreviations indicate the primary purpose in 1977 for which water from the well was used:

$\begin{array}{llll}\text { ARCD } & \text { air conditioning } & \text { IND } & \text { industrial } \\ \text { COM } & \text { commercial } & \text { IRR } & \text { irrigation } \\ \text { DOM } & \text { domestic } & \text { OTHR } & \text { other } \\ \text { F.P. } & \text { fire protection } & \text { P.S. } & \text { public supply } \\ \text { INST } & \text { institutional } & \text { UNSD } & \text { unused }\end{array}$

Use of Well

The following abbreviations indicate the principal use of the well in 1977 or the purpose for which the well or hole was drilled.

$\begin{array}{llll}\text { DEST } & \text { well destroyed } & \text { UNSD } & \text { well unused } \\ \text { OBS } & \text { observation well } & \text { WTDR } & \text { withdrawal of water } \\ \text { RECH } & \text { recharge water } & & \end{array}$

\section{Depth of Well}

The figures give well depth, in feet below land surface.

\section{Screen Setting and Total Screen Length}

The altitude of the top and bottom of the screened interval is given in feet above or below (-) National Geodetic Vertical Datum of 1929. Total length of screen or perforated pipe in that interval is given, in feet. In some wells, screen was set at two or more intervals; in such cases the difference between the altitudes of the two screen settings is different from the total screen length.

\section{Diameter of We11}

The diameter of the well is the nominal inside diameter of the smallest or innermost casing at land surface, in inches.

\section{Water Level (feet below land-surface datum)}

The water level given is the reported original static water level, in feet above or below land surface, when the well was completed.

\section{Date of Measurement}

The date of water-level measurement is given by month (M), day (D), and year $(Y)$. 


\section{Lift Type}

The following abbreviations indicate the type of pump or other conveyance known or assumed to have been used in 1977 to bring water to the surface.

$\begin{array}{llll}\text { CENT } & \text { centrifugal } & \text { TURB } & \text { turbine } \\ \text { JET } & \text { jet } & \text { NONE } & \text { no pump in well } \\ \text { SUBM } & \text { submersible } & \text { OTHR } & \text { some other type of lift }\end{array}$

\section{Aquifer Developed}

The following abbreviations indicate the hydrogeologic unit that yields water to the we11. Where two or more units yield water to the well, the probable principal unit is given.

\section{GLACIAL Pleistocene and (or) \\ Cretaceous aquifers undifferentiated}

UPGLAC Upper glacial aquifer

GARD Gardiners Clay

JAMECO Jameco aquifer

\author{
PTWCU Port Washington confining unit \\ PTWAQ Port Washington aquifer \\ MAGOTHY Magothy aquifer \\ RARITAN Raritan confining unit \\ LLOYD Lloyd aquifer
}

\section{Specific Capacity}

The value in this column is the number of gallons per minute pumped from the well per foot of drawdown in the well, as reported by drillers.

\section{Abbreviations}

$\begin{array}{llll}\text { COORD } & \text { coordinates } & \text { LSD } & \text { land surface datum } \\ \text { D } & \text { day } & \text { M } & \text { month } \\ \text { DIAM } & \text { diameter } & \text { MEAS } & \text { measurement } \\ \text { FT } & \text { feet } & \text { NGVD } & \text { National Geodetic Vertical } \\ \text { GPM/FT } & \begin{array}{l}\text { gallons per minute pumped per } \\ \text { foot of drawdown in the well }\end{array} & \mathrm{Y} & \text { Datum of 1929. } \\ & & \end{array}$

\section{Map Coordinates}

Locations of wells are given by map coordinates, based on a latitude and longitude grid system, to aid the reader in locating the wells in plate 1 . In this system, 5-minute intervals of latitude are lettered consecutively from south to north, and 5-minute intervals of longitude are numbered consecutively from west to east. The grid coordinates are shown along the margins of plate 1. 



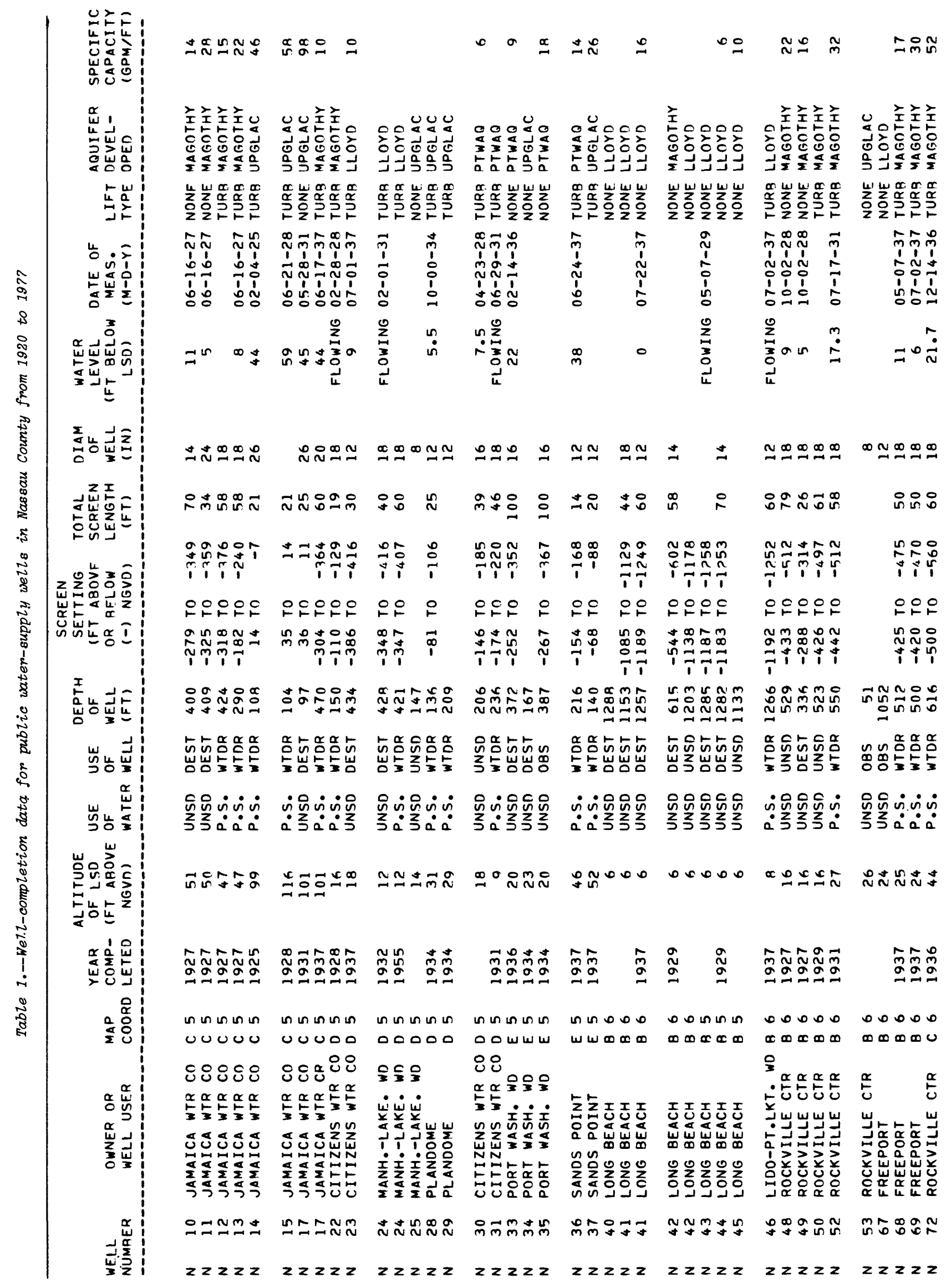




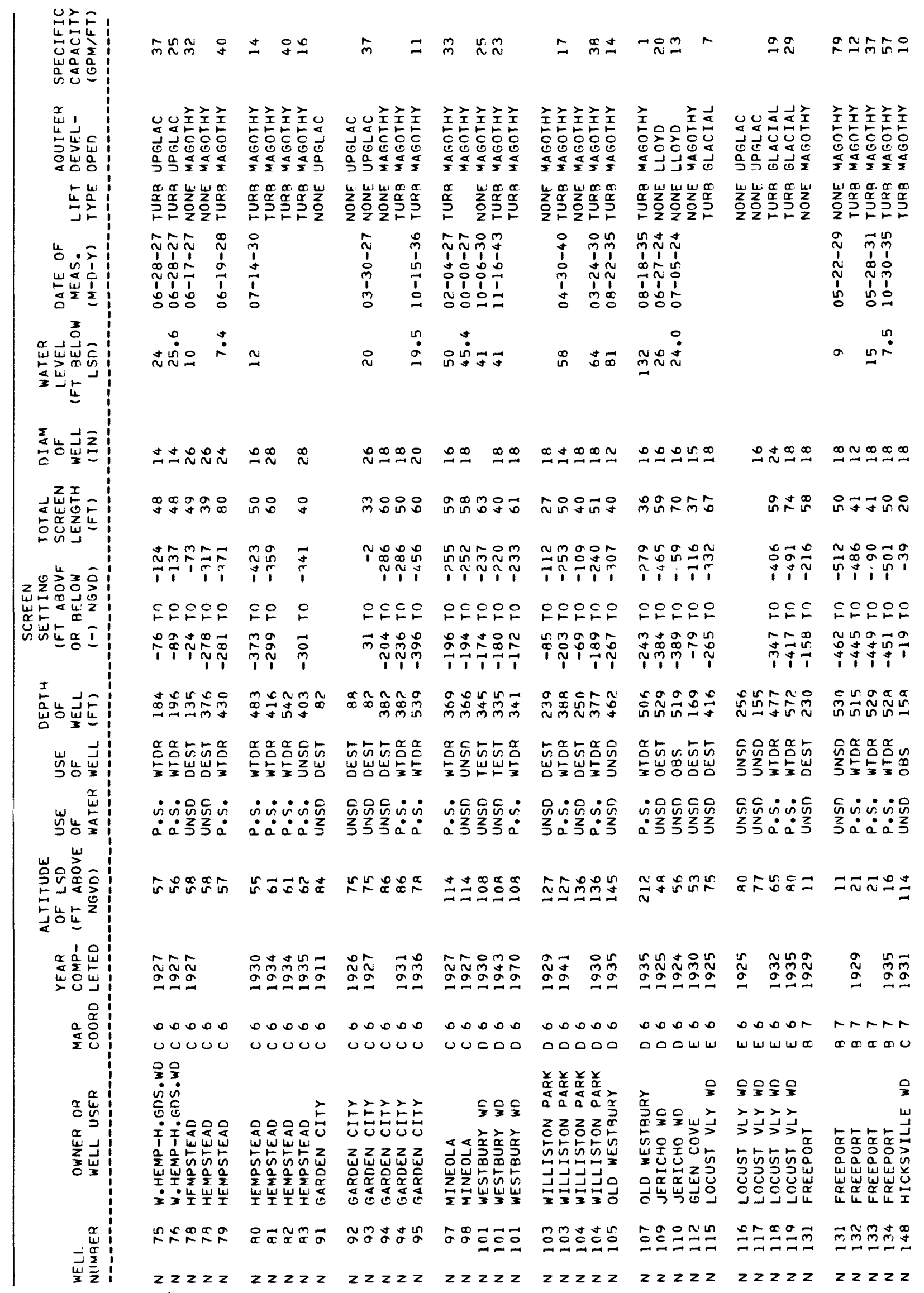




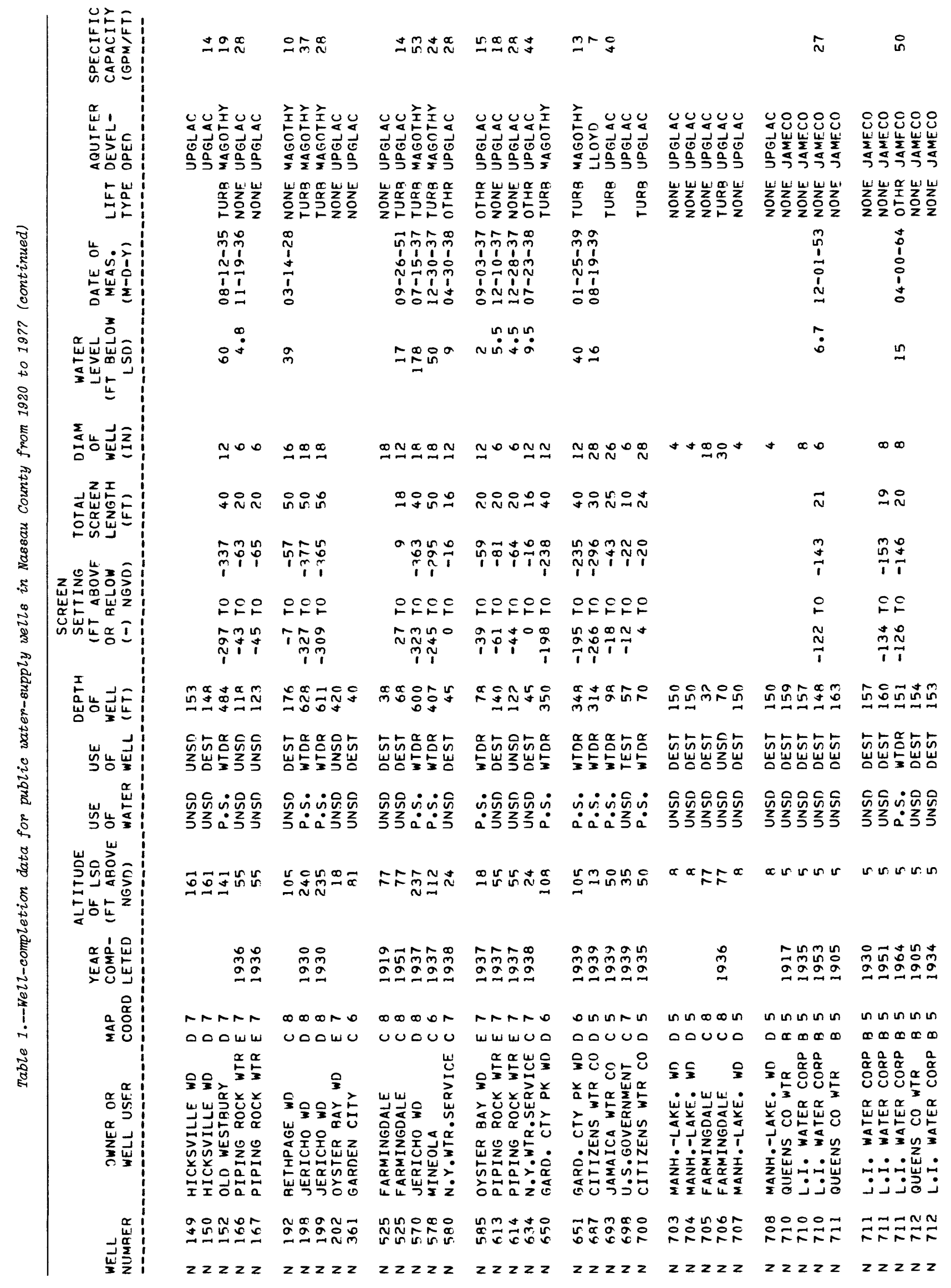




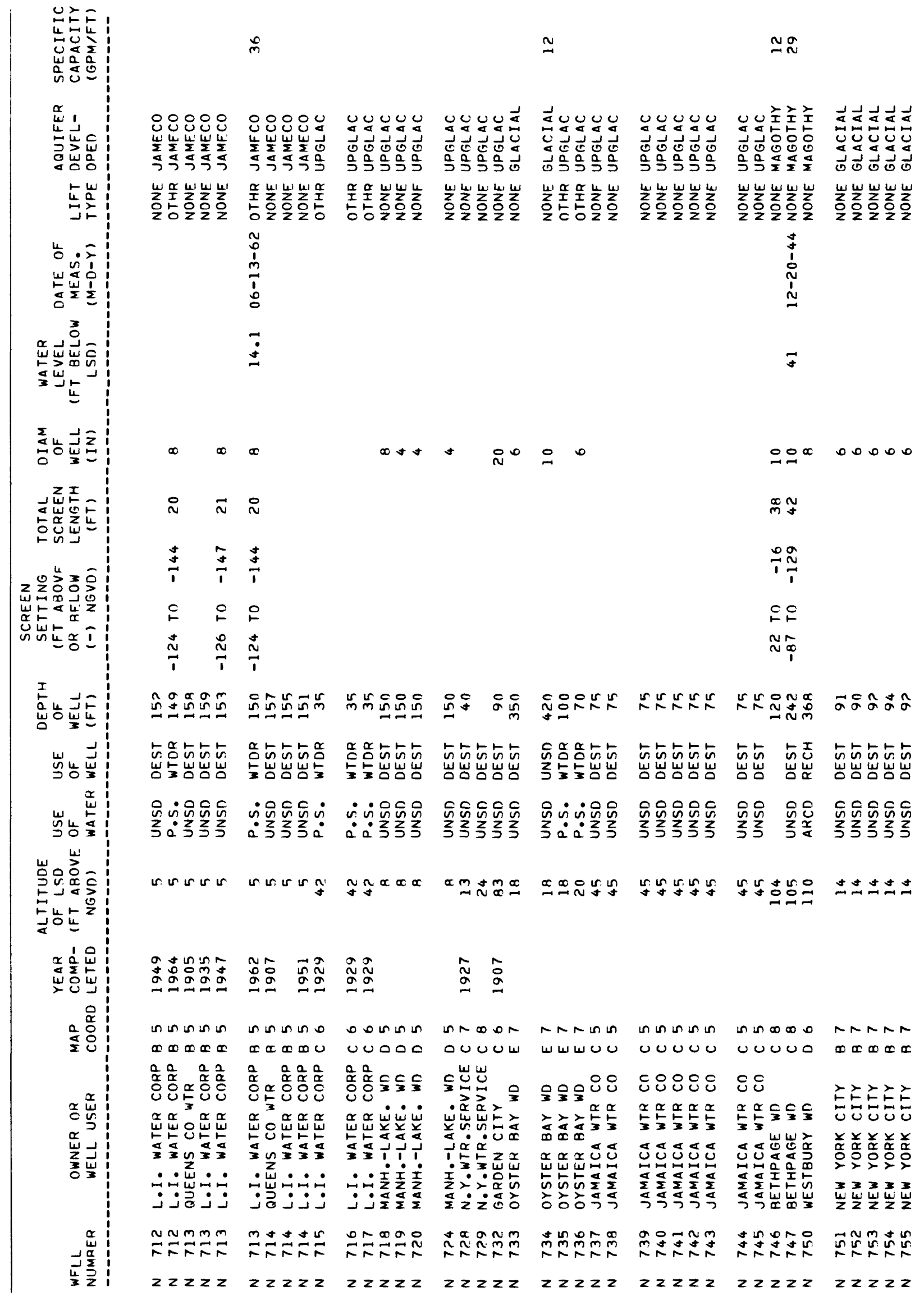




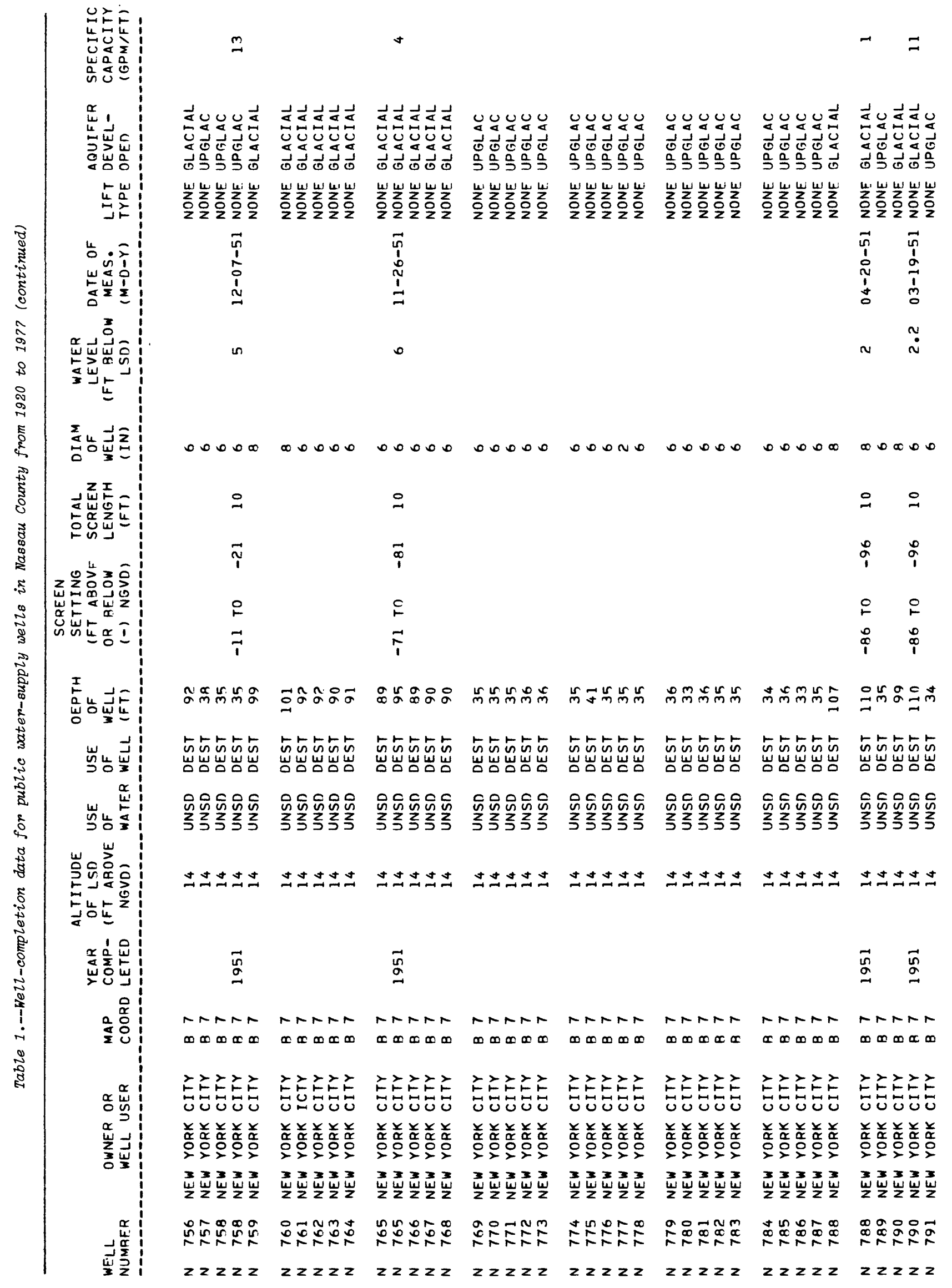




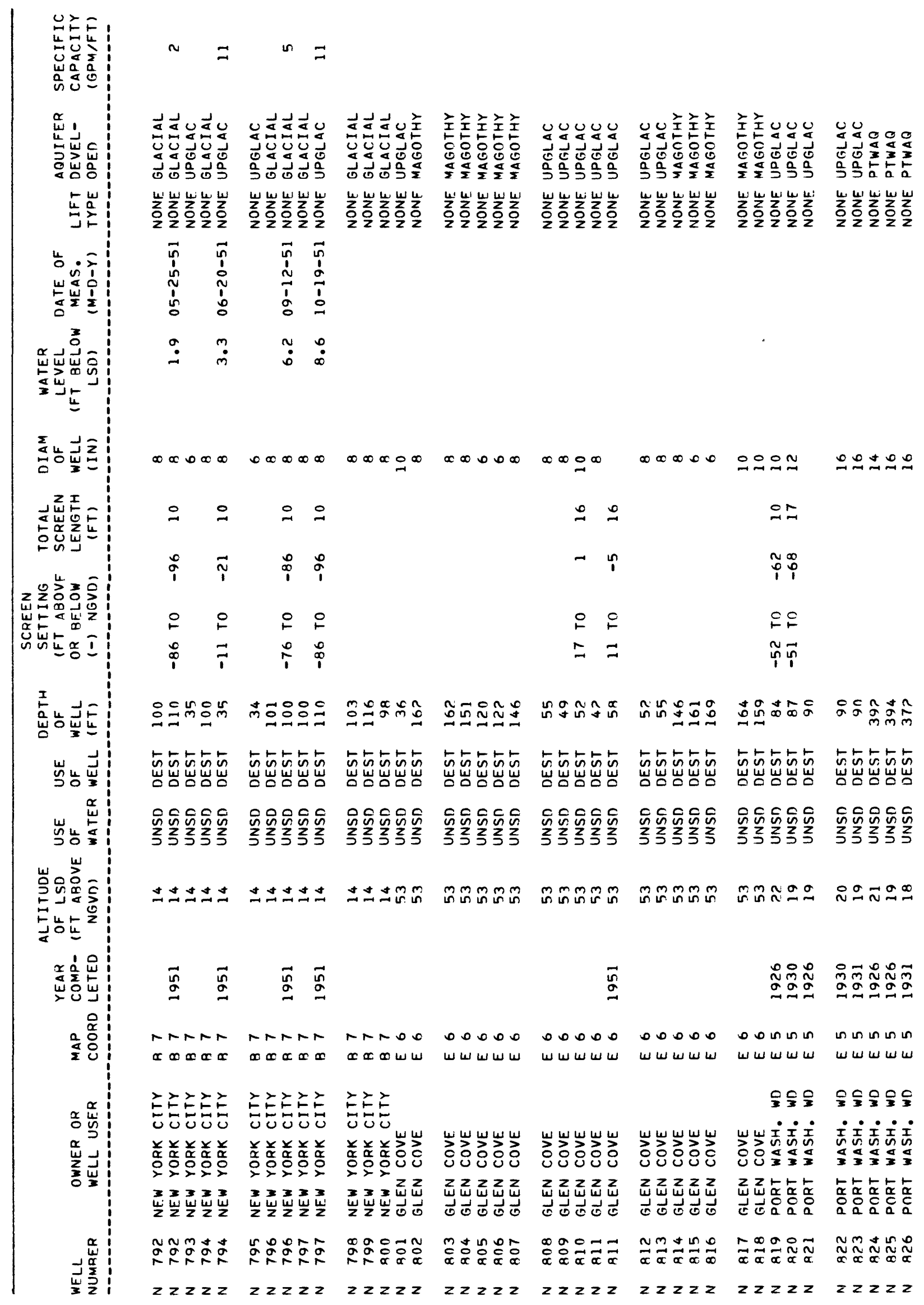




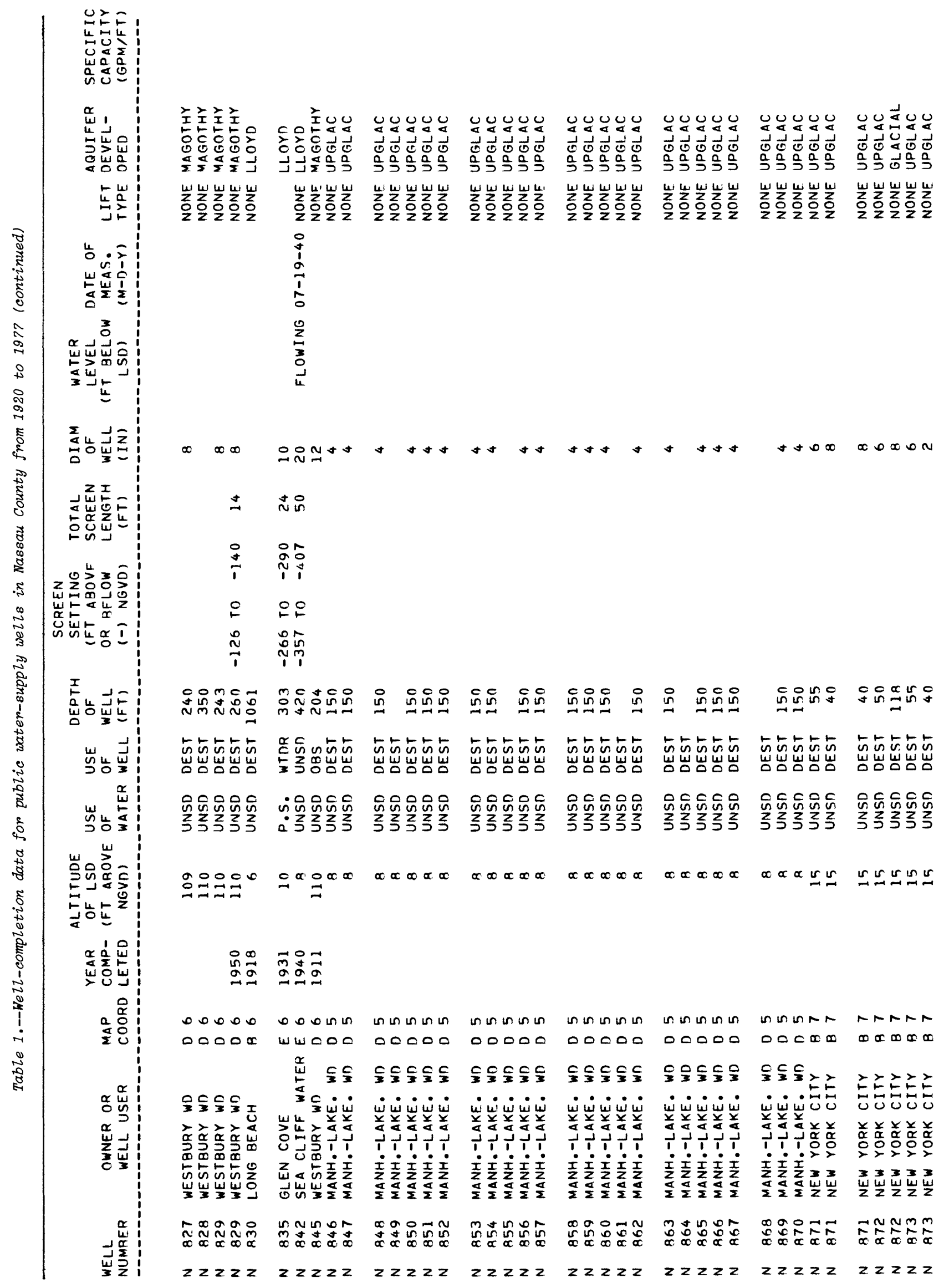




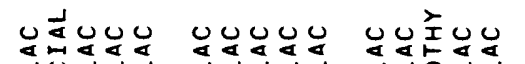

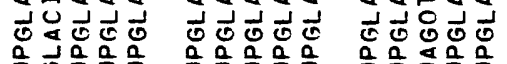

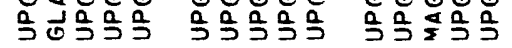

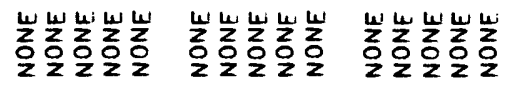

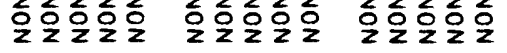
눙 ₹ 山这 造岁要

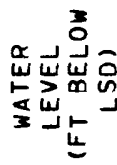

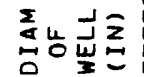

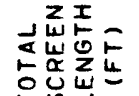
z ํํㅇํํㅇำ

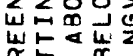

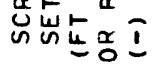
妥岂岂点 岁告峌

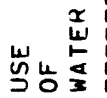
岸崖

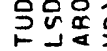

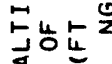
年

$\circ \infty \infty \infty$ 00000 $0 \infty \infty \propto$ 으은용

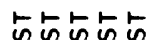
峆岲敋出

らピらら 岁詮㛧岩

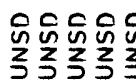

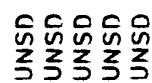

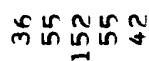

ヒららららら 岁岁骀岩山

\section{으ำ जิ जิ}

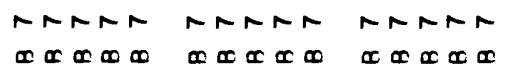
$\infty \circ \infty x$ 0000 $\infty \infty \infty \infty$ 00000 00000

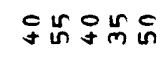

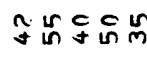

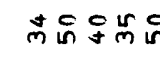

ㅇํㅇ유의

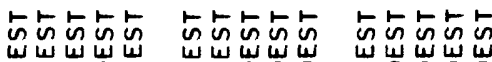

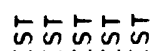

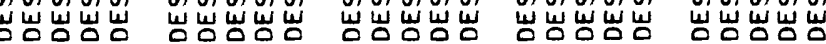

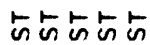

ทิติ ติติ

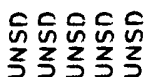
ทํํํํํํํํํํํำ

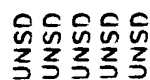

ติํํํํํํำ

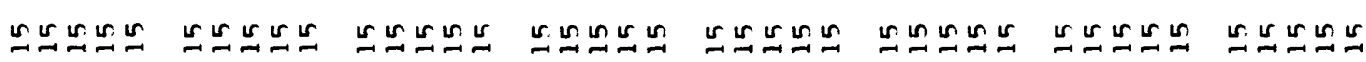

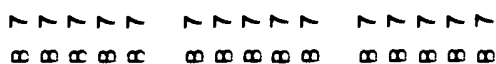

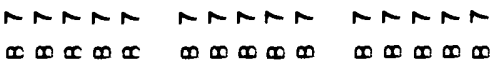

$\infty \propto \propto \infty \infty$ $\propto \propto \infty \propto ⿻$

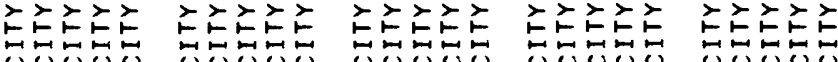

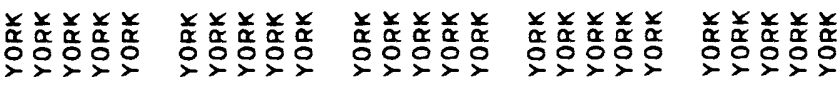

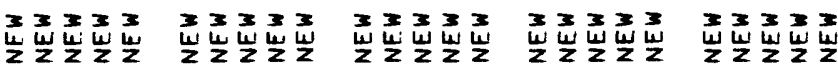

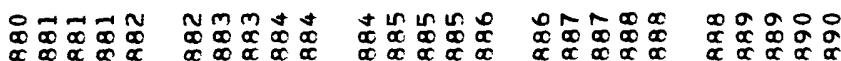
$z z z z z z z z z$ zzzz zzzzz zzzzz 


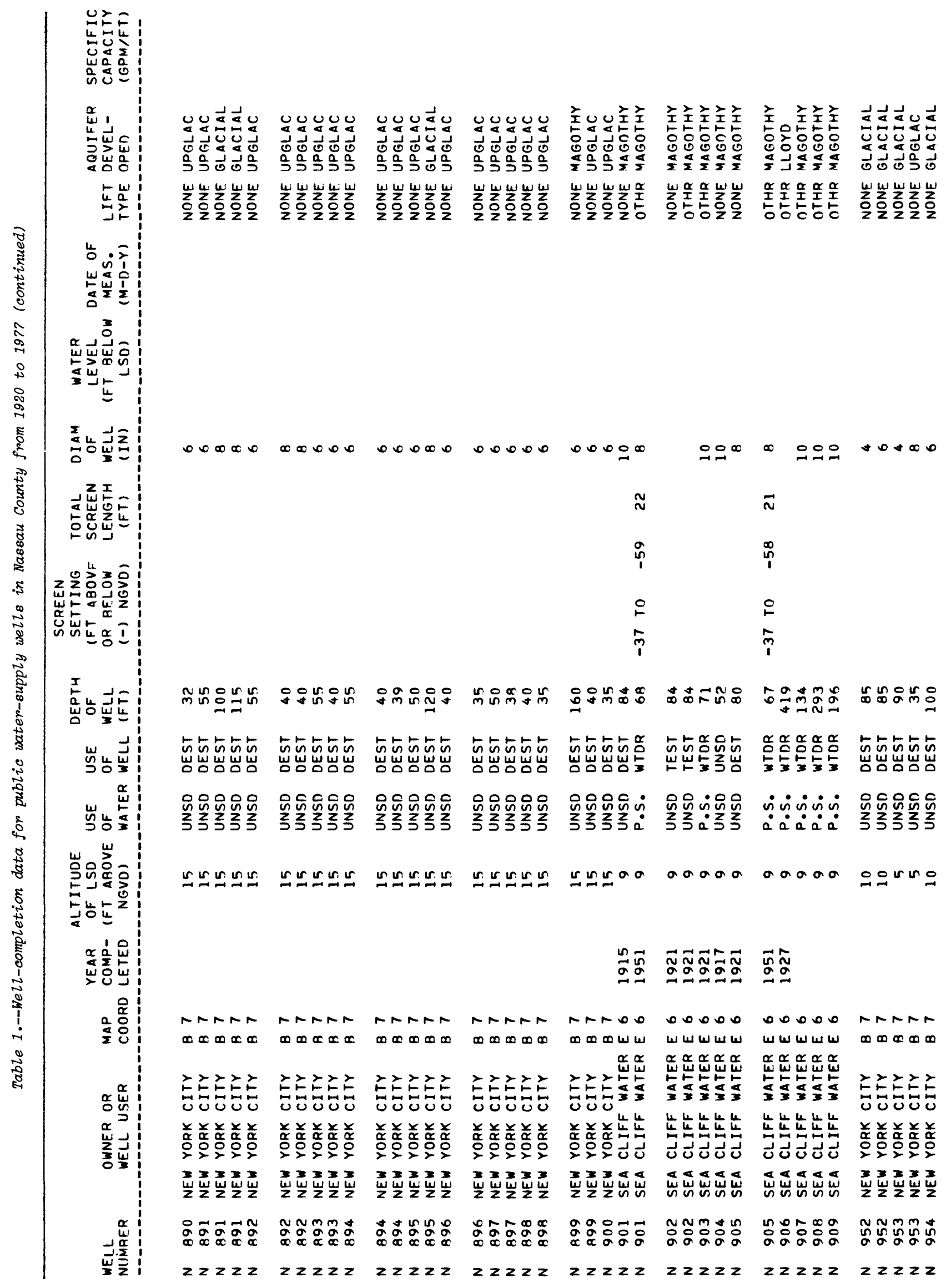




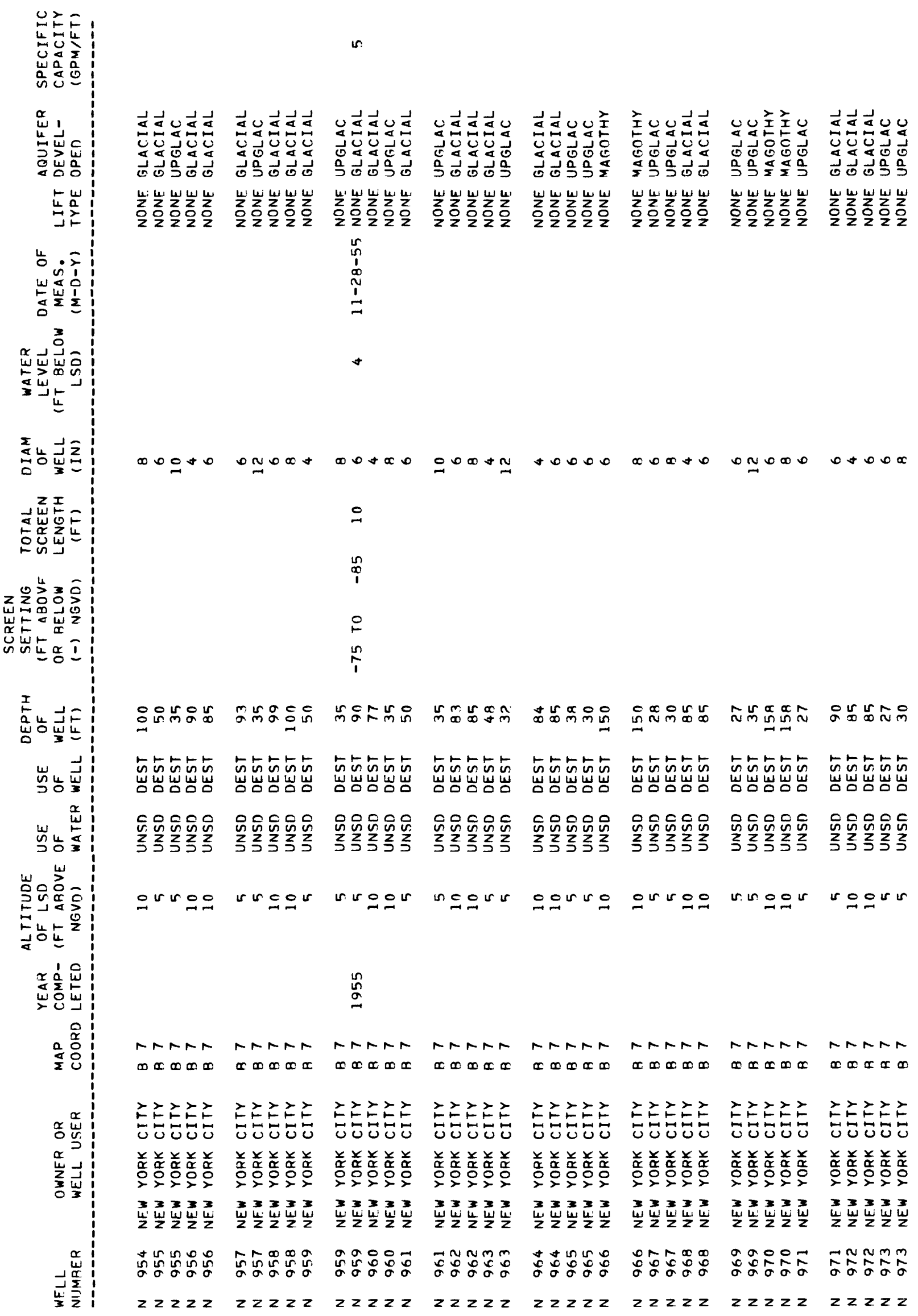




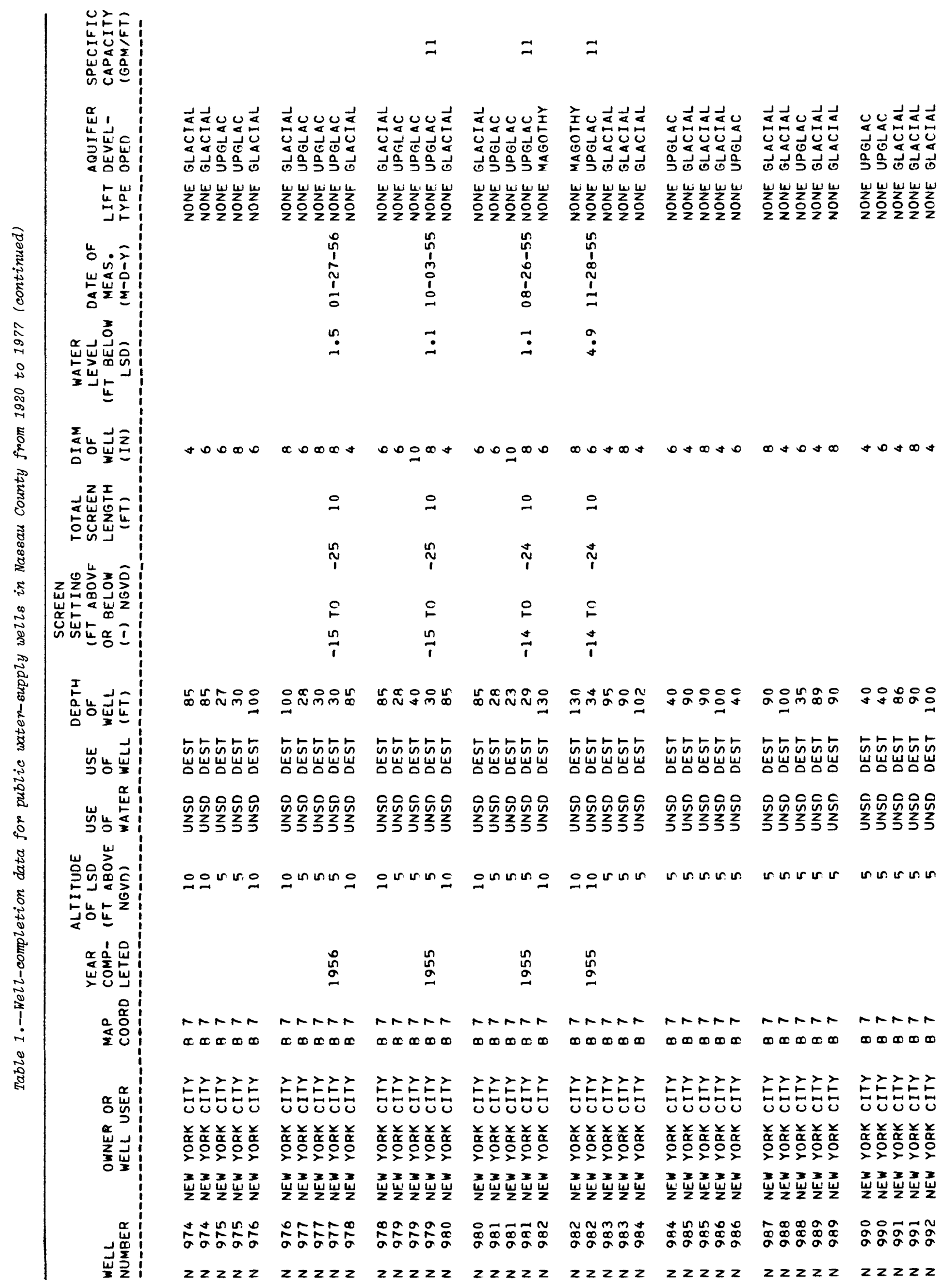




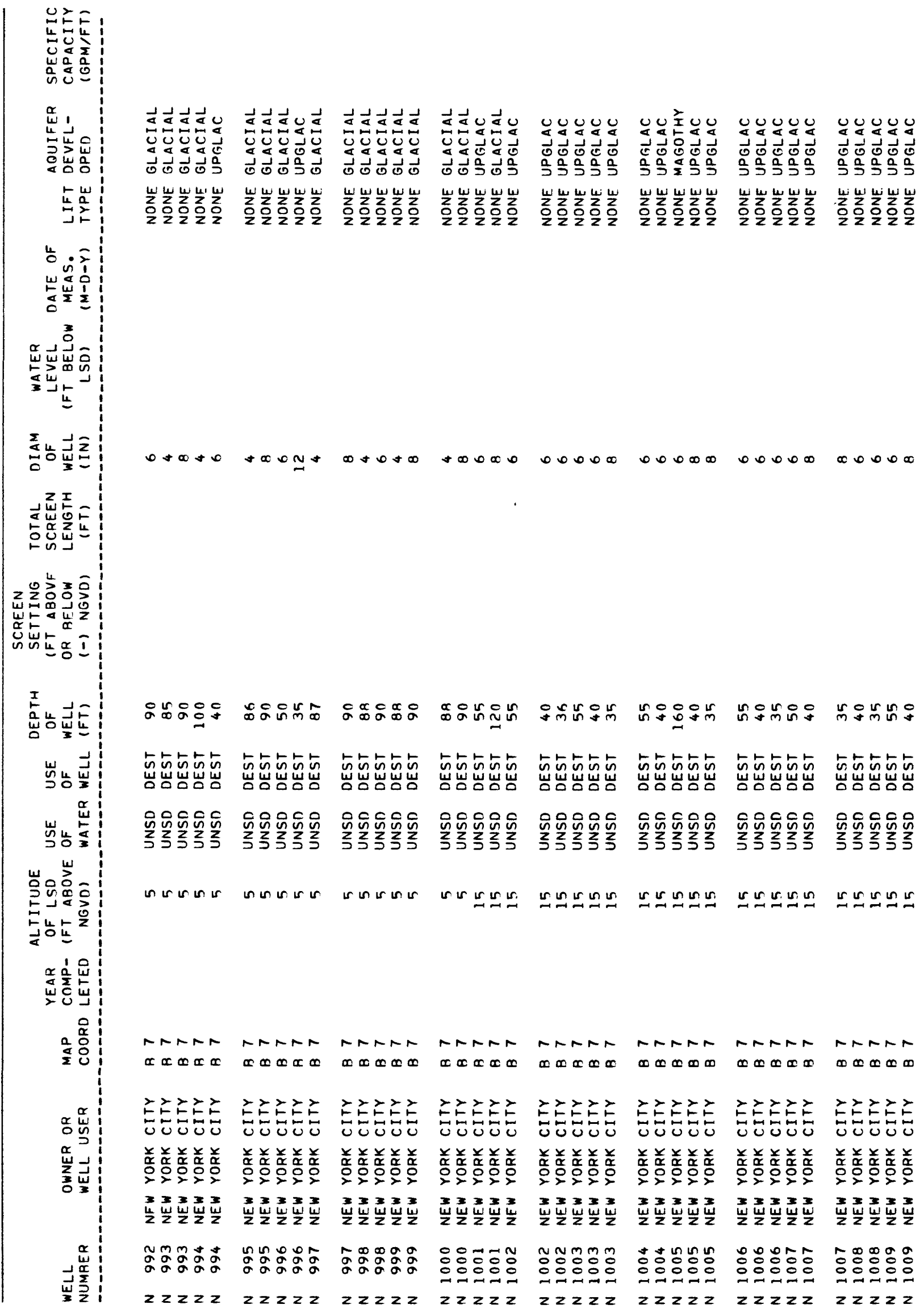




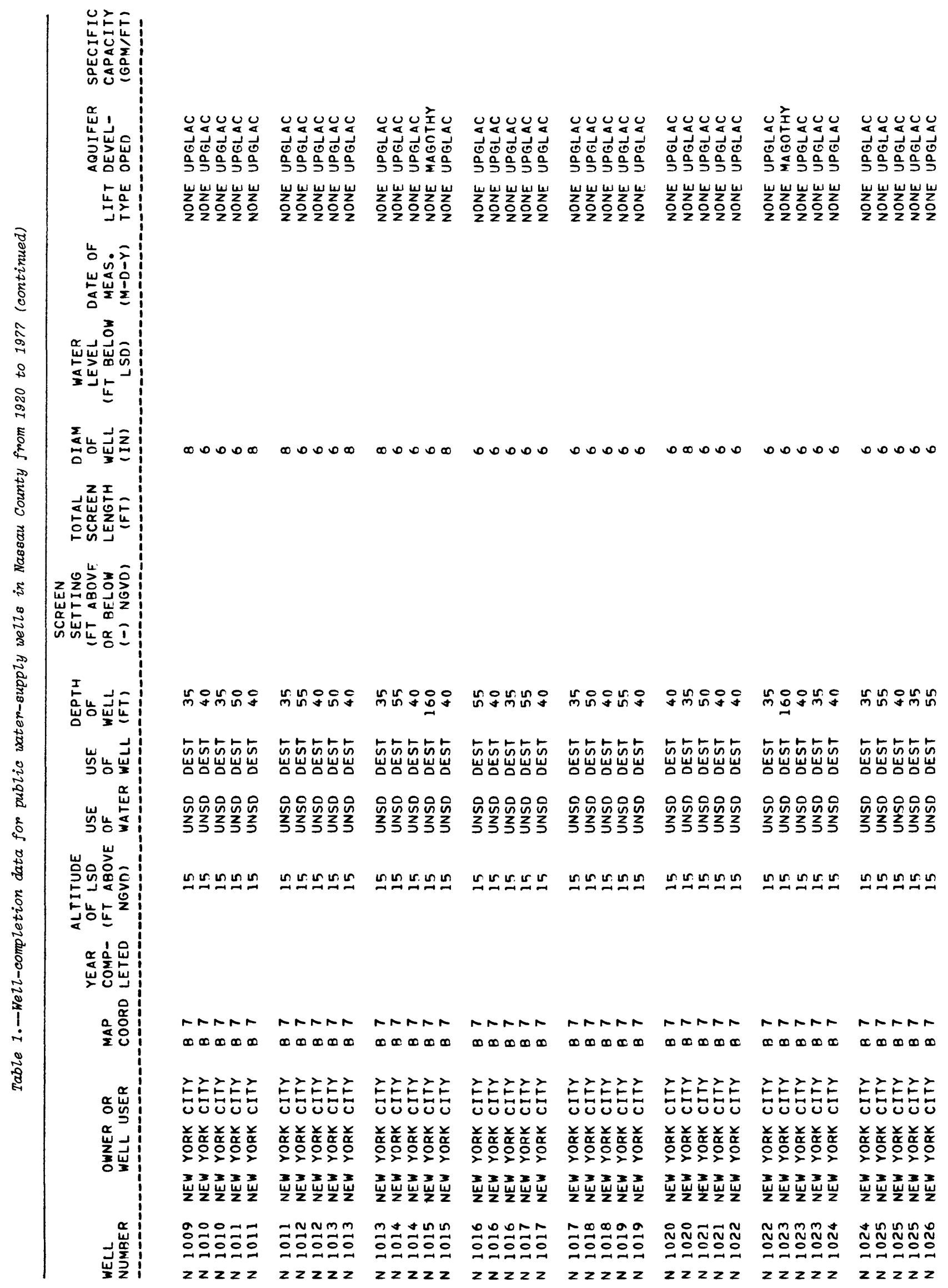




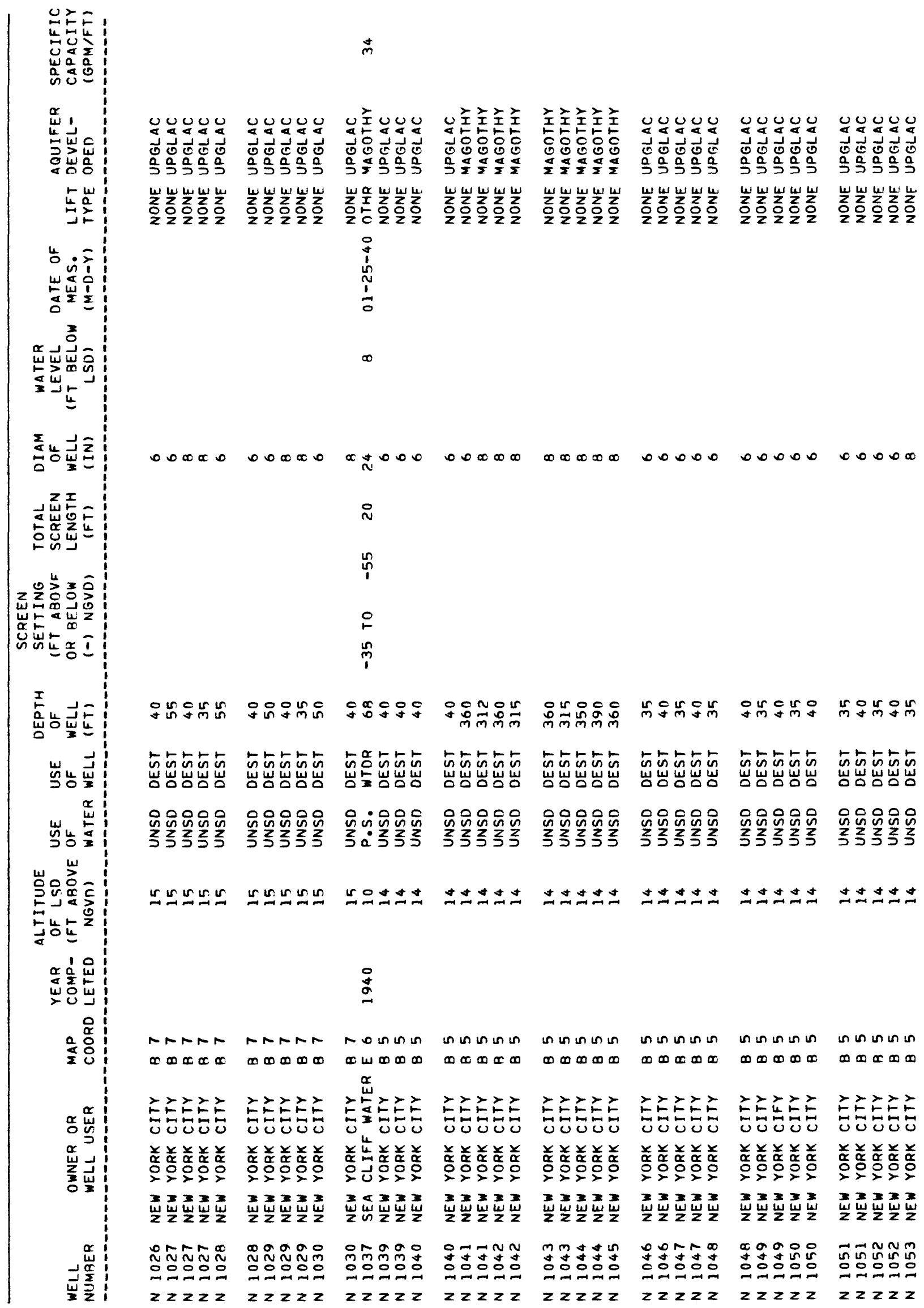




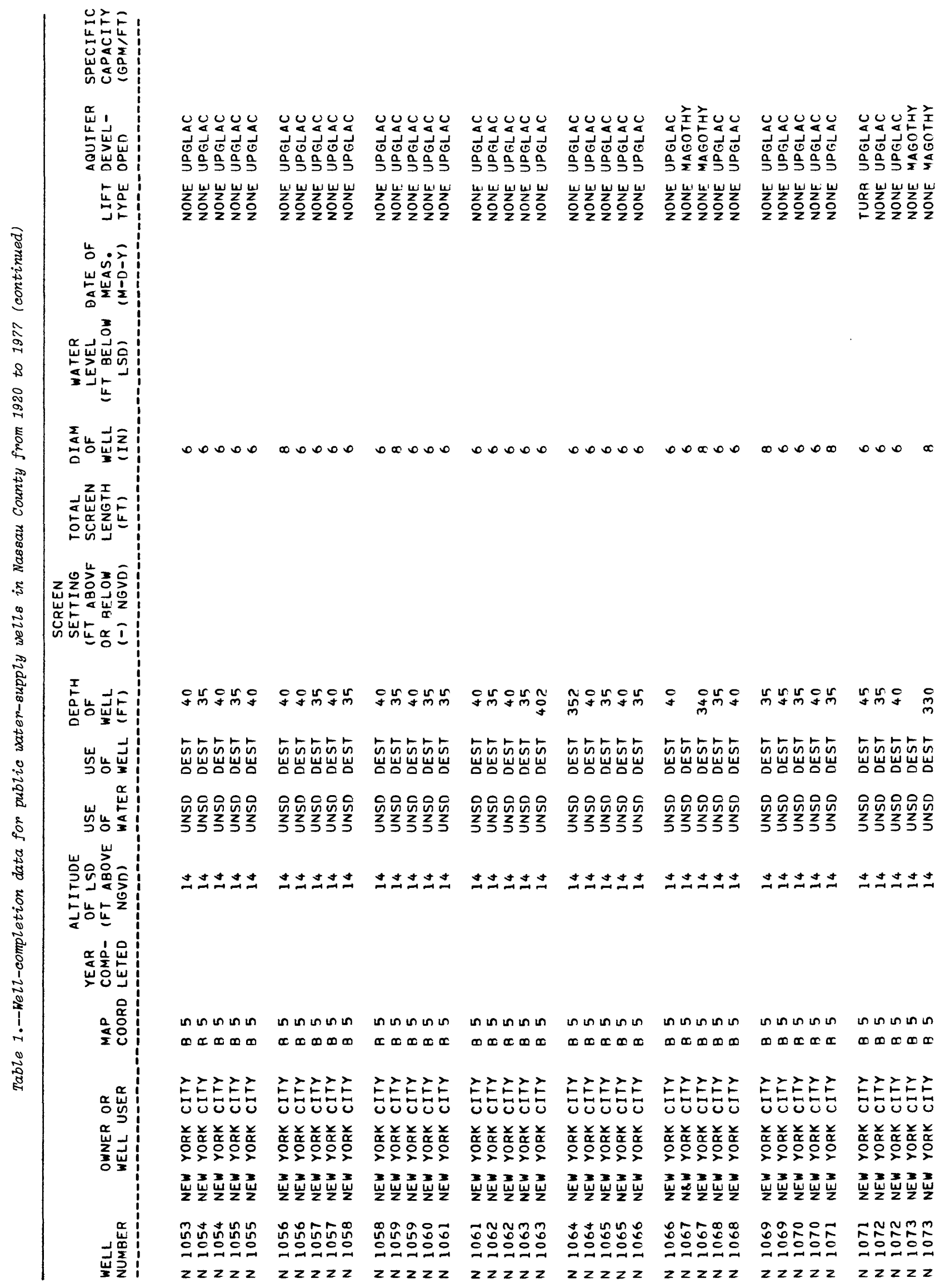




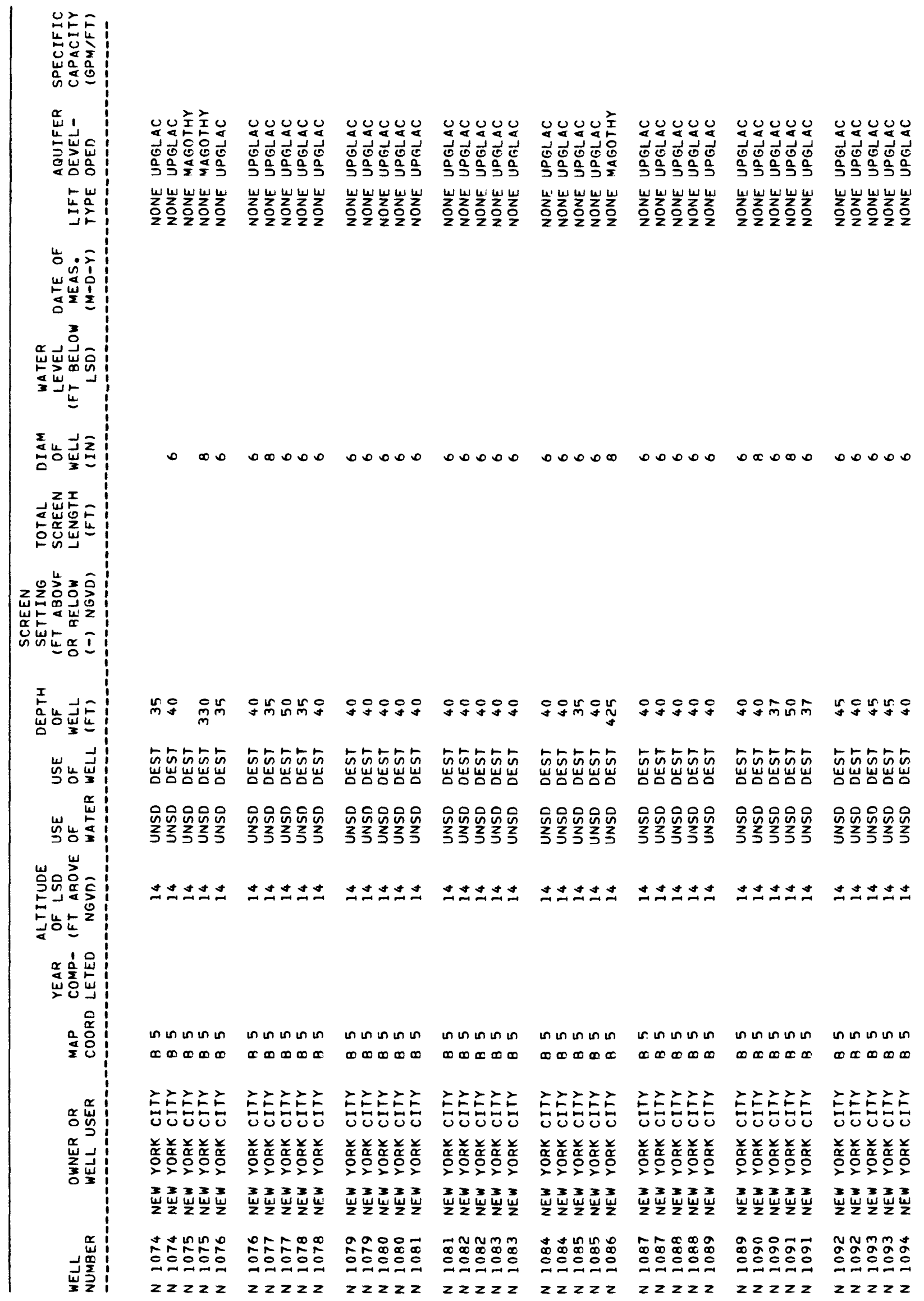




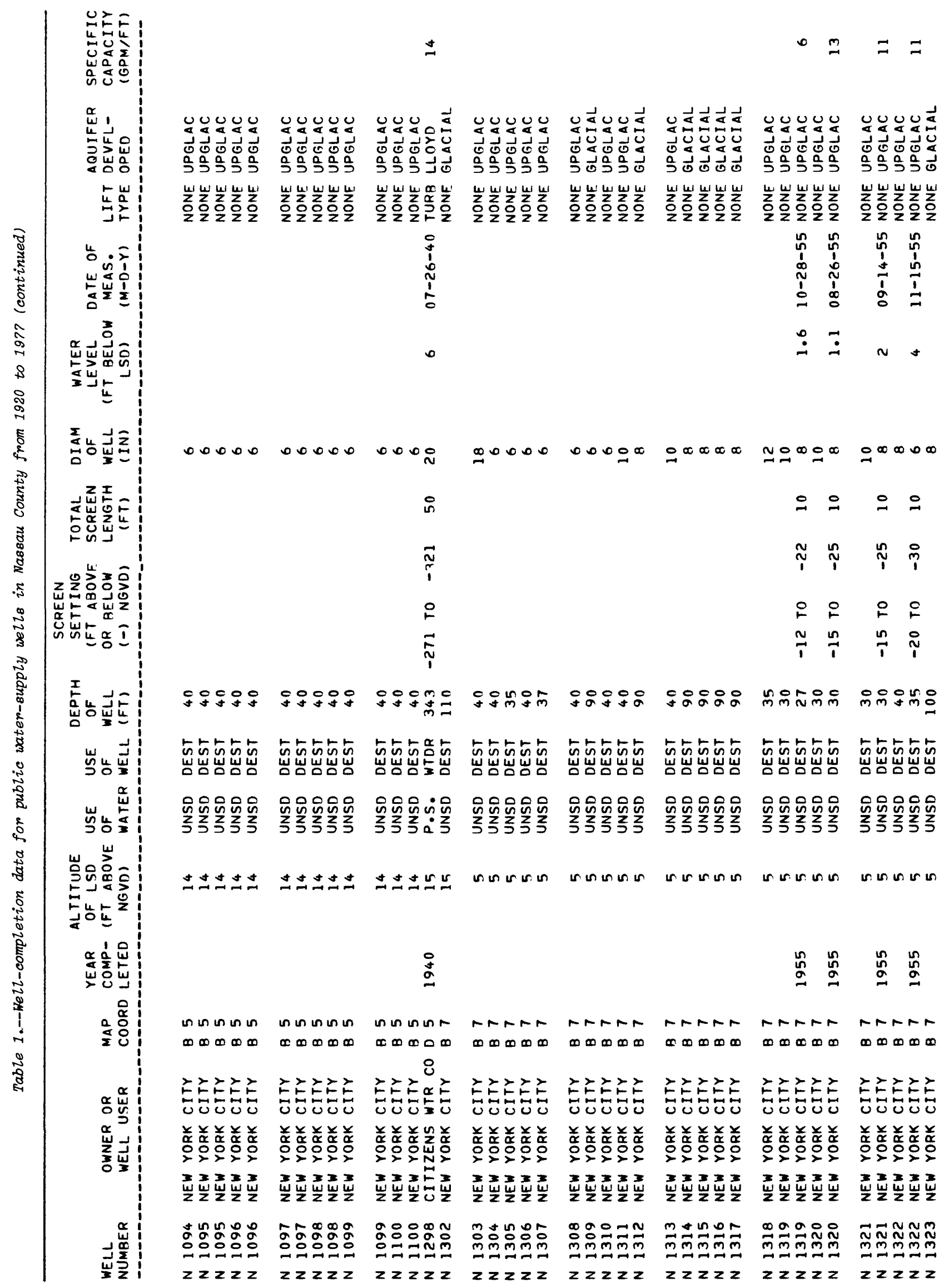




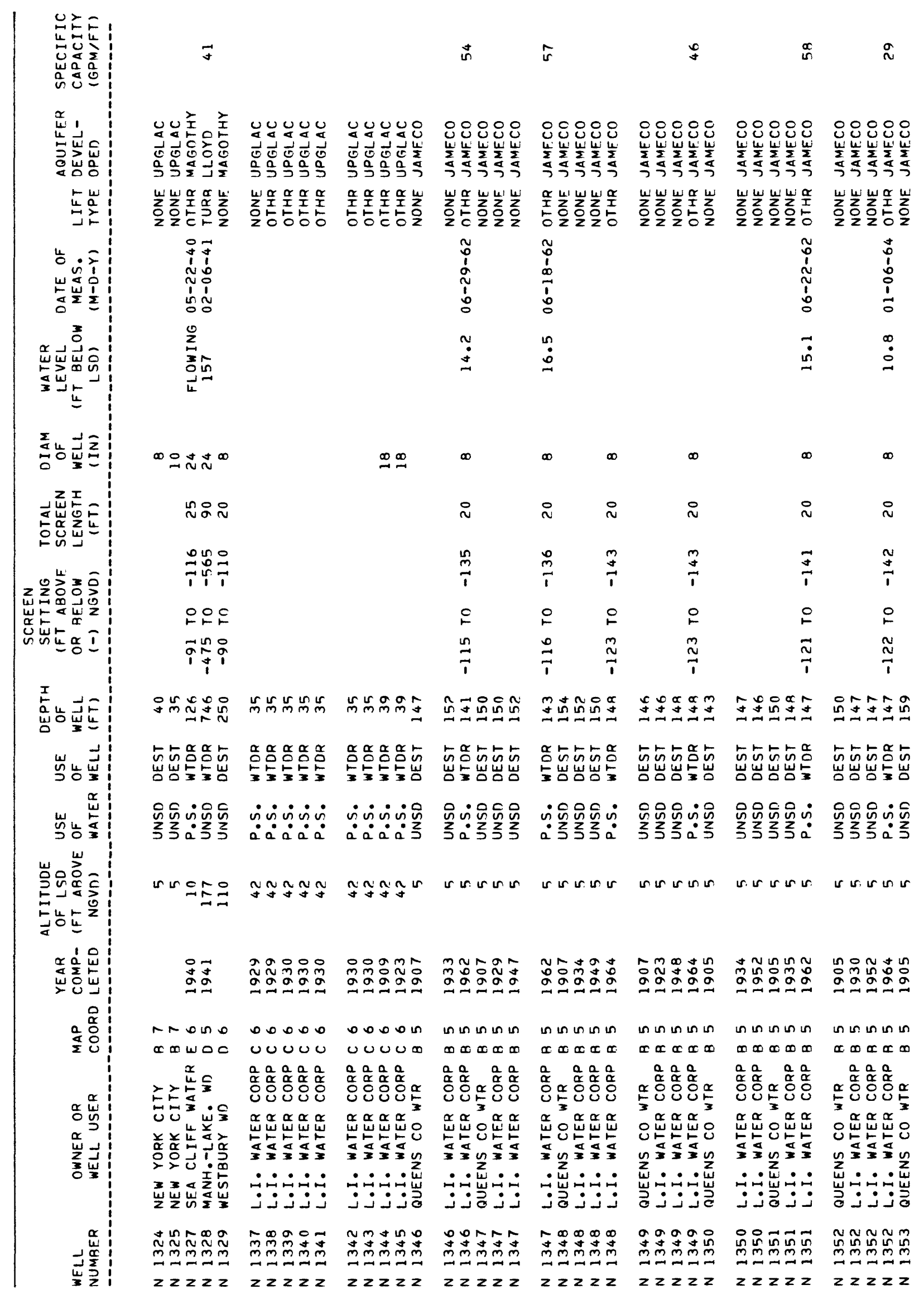




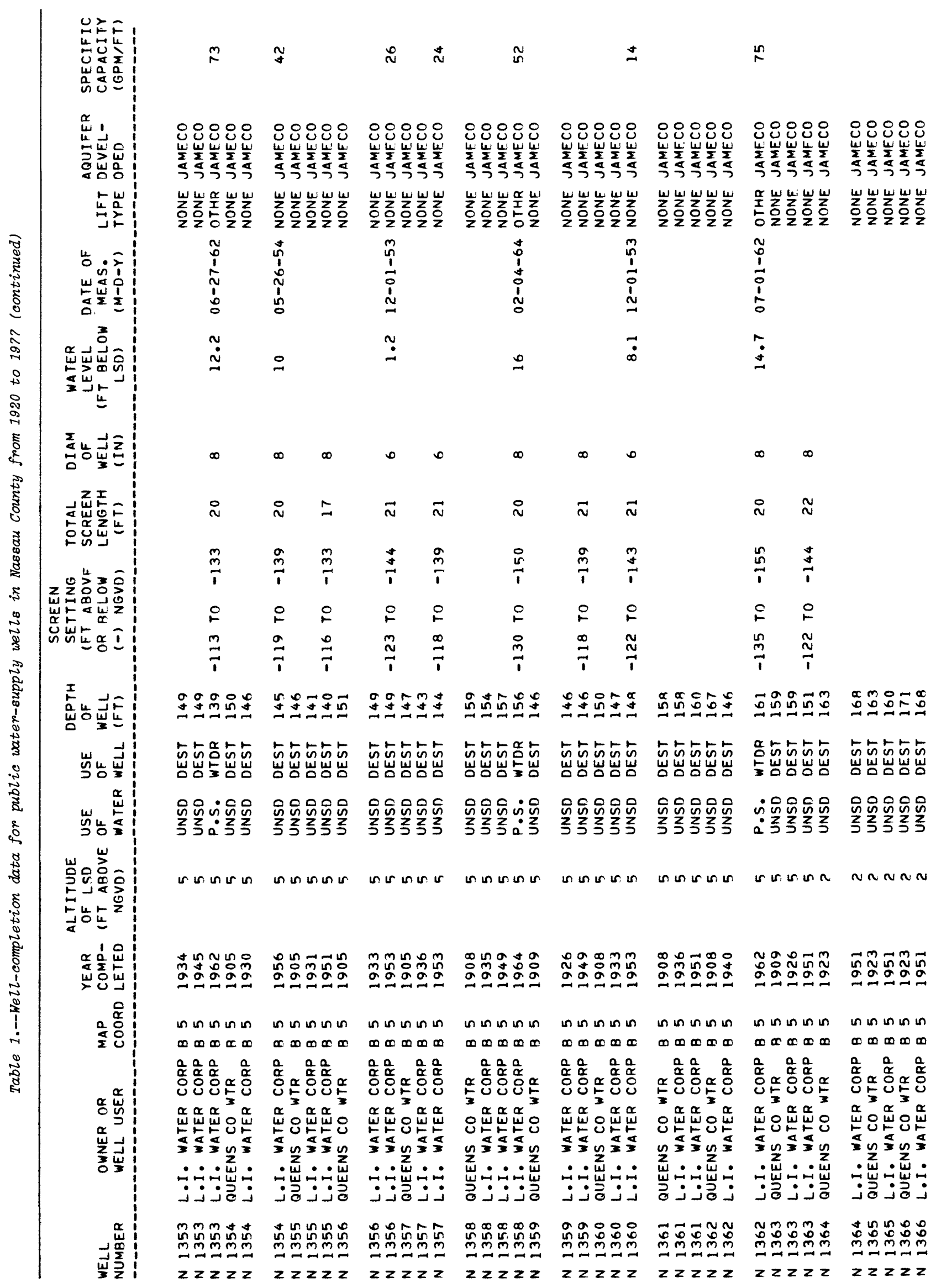




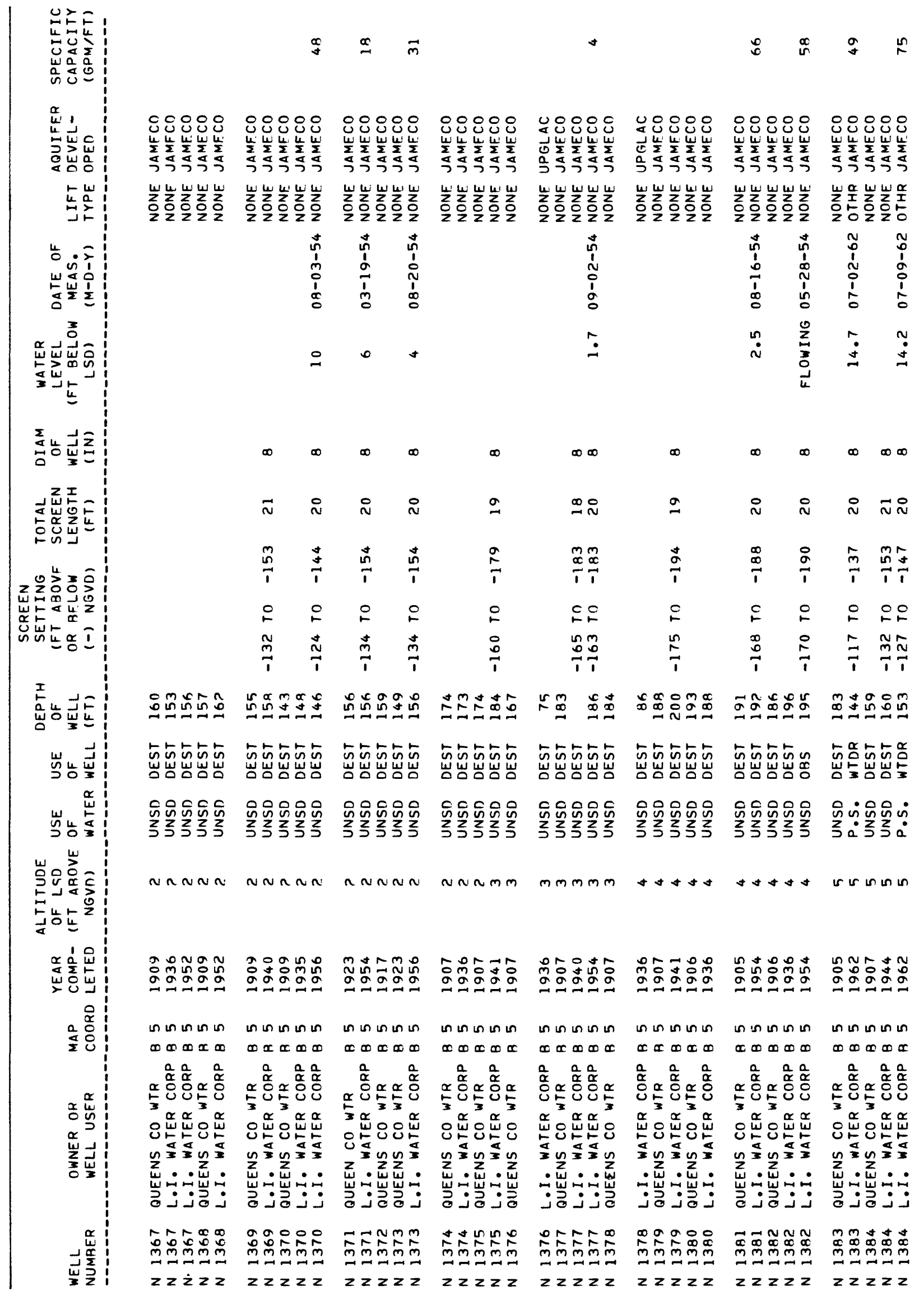




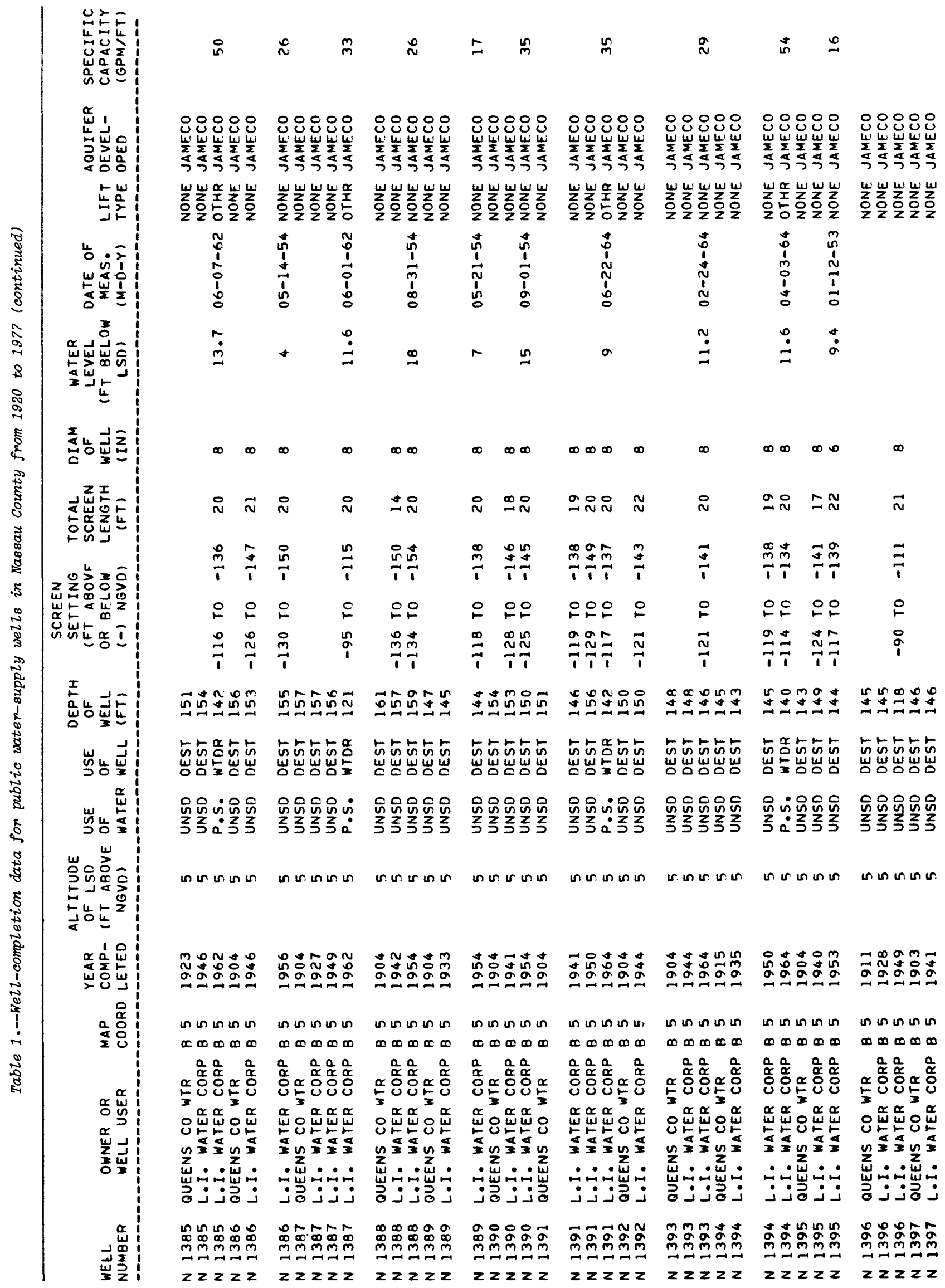




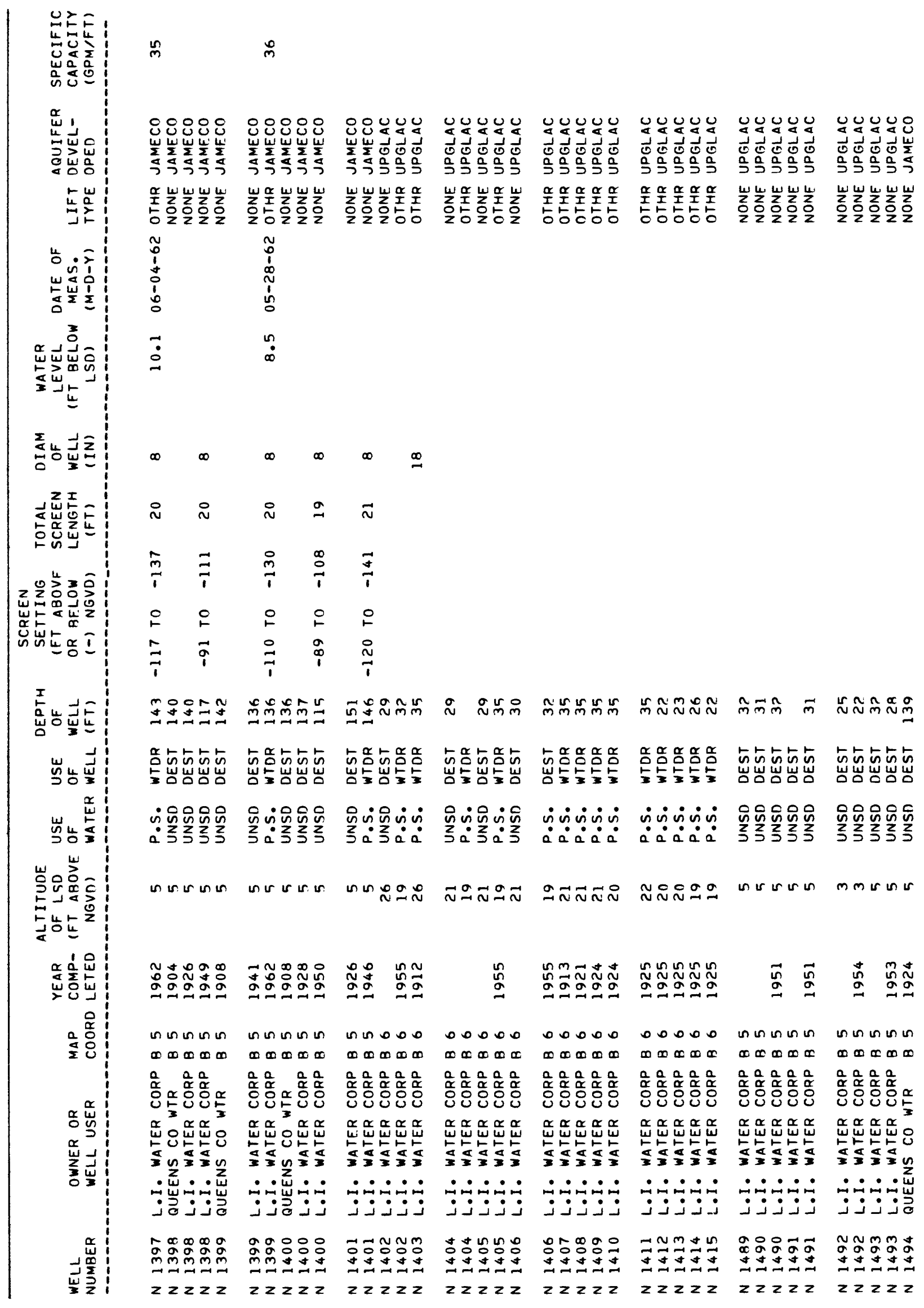




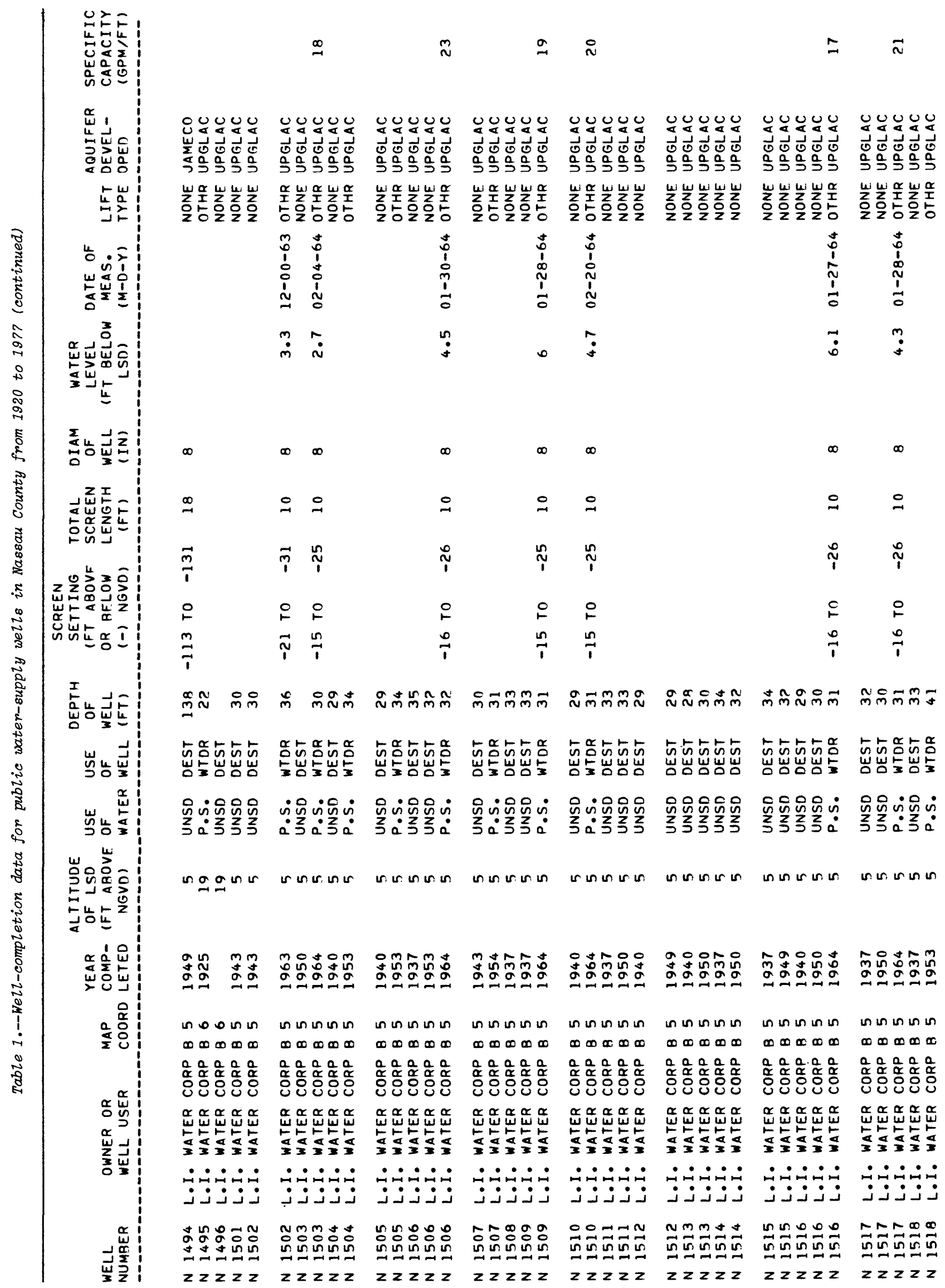




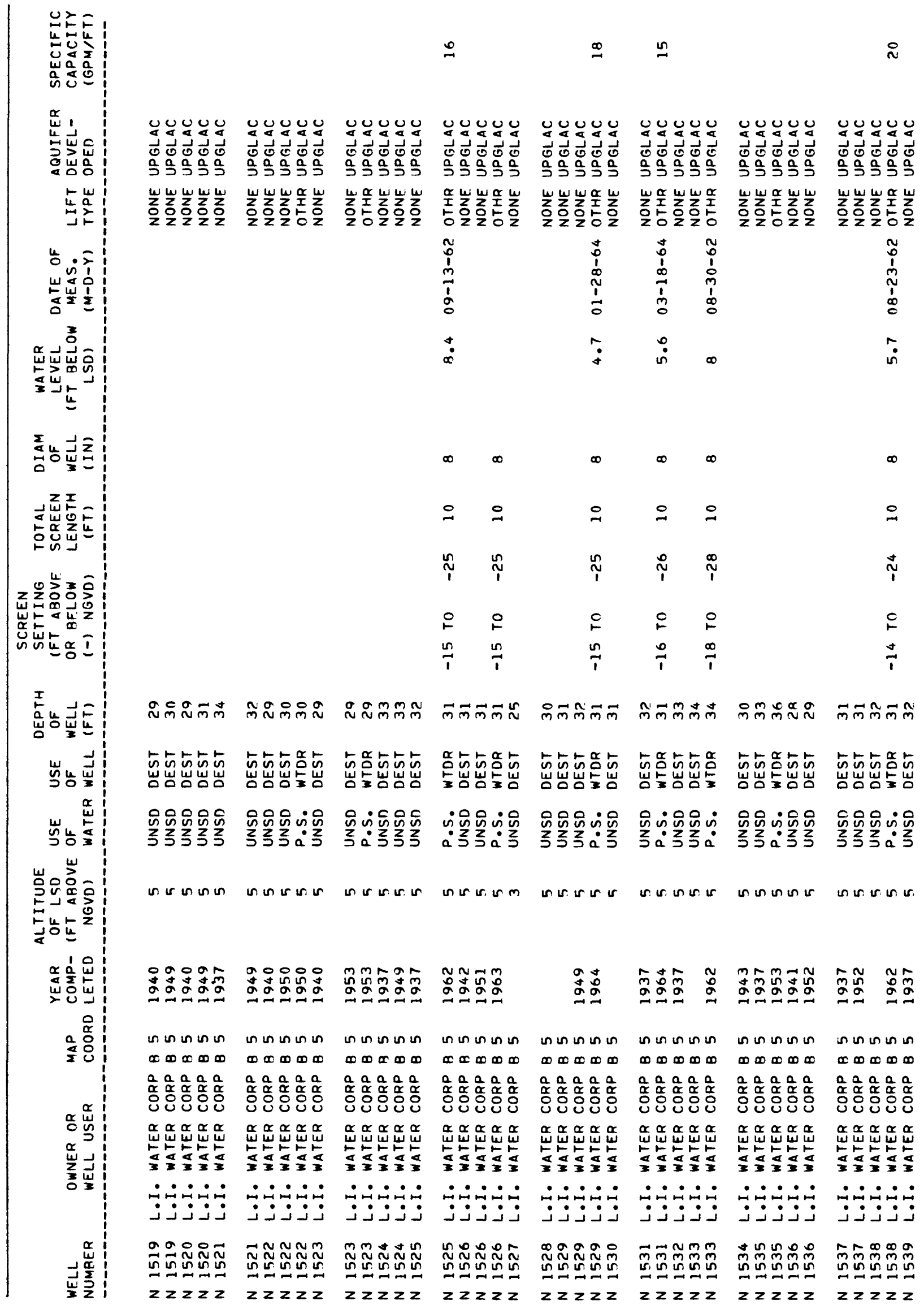




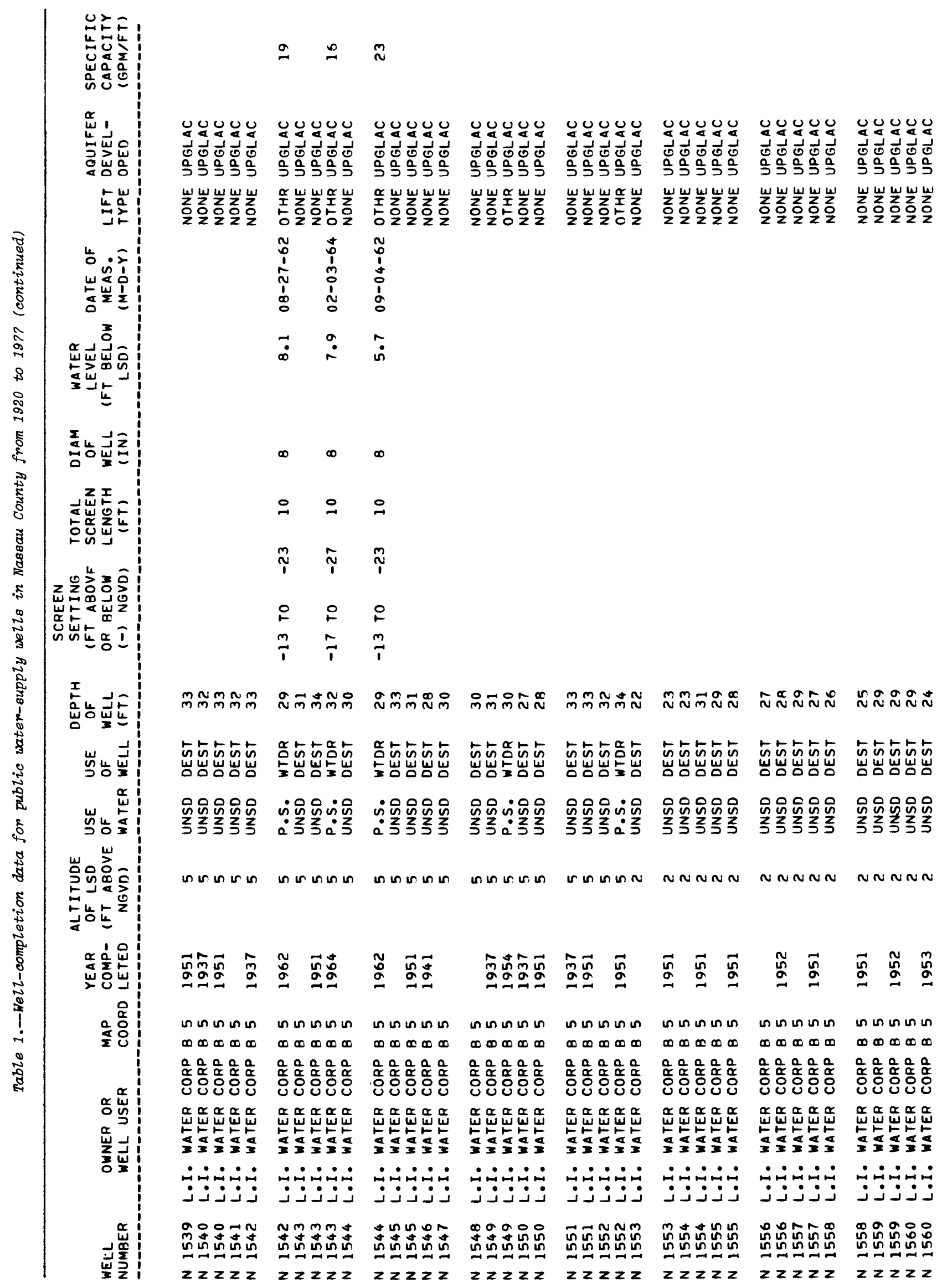




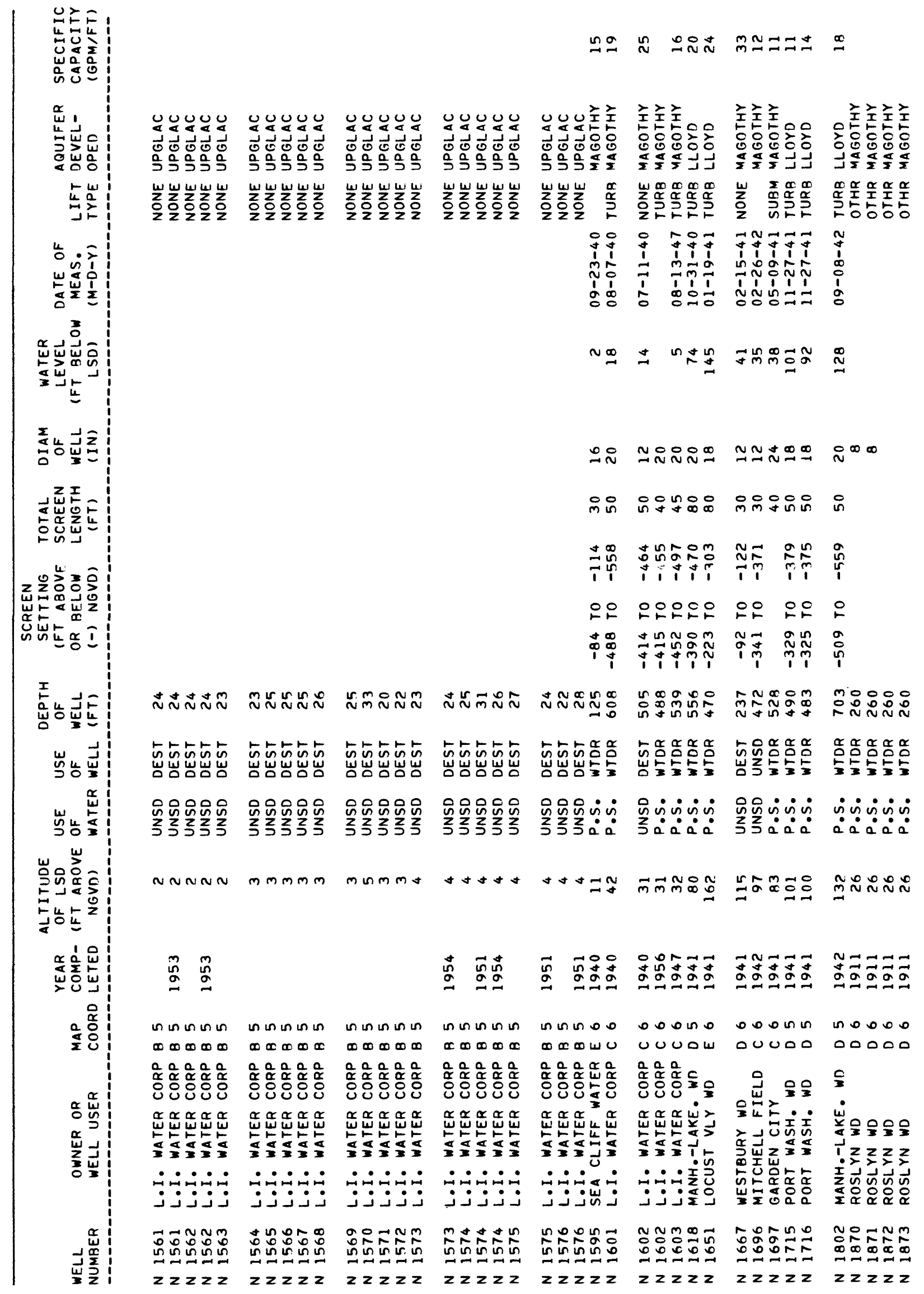




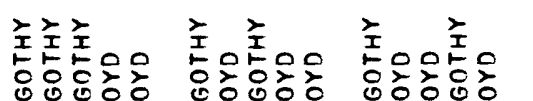

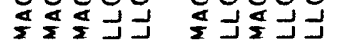

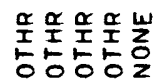

岂岩岩岩

0
1
1
0
1
0
0
0
2
3
0
14

음요요

0000

$0000000 \infty 0$

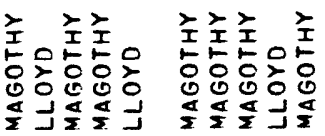

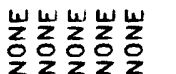

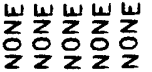

衣主主

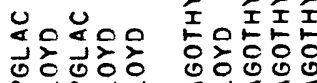
过」范」 过

楸岸崖 岂岂崖崖

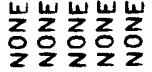

iziziz

文隺是

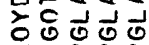

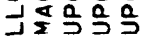

岂乎岂岩岩 之年定

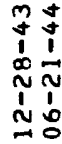

袋色

$\simeq \simeq \infty \infty$

$8 \stackrel{0+2}{n}$

운

$T$

오운

욘ำ

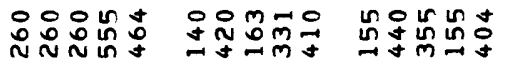

กี่芷品紫

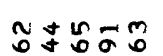

:๐ㅗำ

aromis $T$

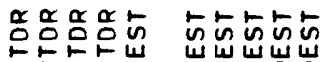

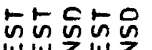

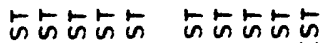

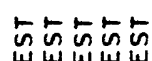

再然三

ํㅜㄴ음요요 ก-

$35 \leq$

峈㛧颉

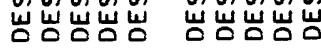

就尔点

稪施尔

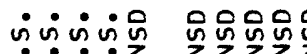

जิ ติ ติที丶⿳一口䒑

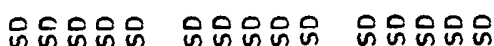

骀㟔崤岩

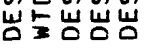

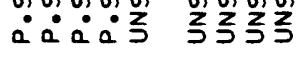

ํㅠํํำ

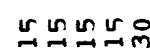

sions un

s

논

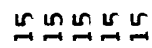

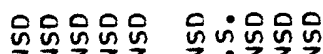

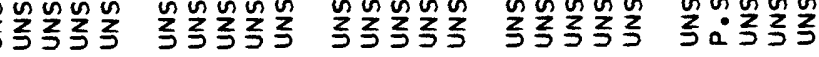

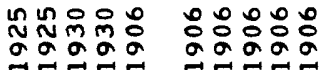

o $000 \mathrm{n}$

०ODO

$$
8
$$

$\stackrel{x}{5}$

오은요는

조전

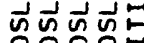

웅워

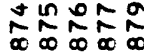

$\geq 2 \geq 2$ in un in in in 00000 원용 $\alpha \alpha \alpha \alpha \alpha$

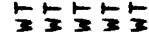

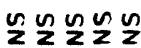
亗亗亗亗 EE⿱E口心E 던드 어에

z z z z Z
용ํㅇ

영응

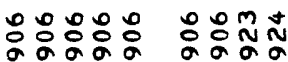

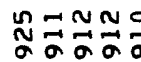

임워 ప

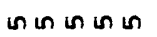
00000

⿰纟勺 $\cos$ in 00000

in in in in in

in in inin in 00000

in in in in in 00000 88888 웡요 웅용 영용 문문문문

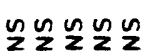
嵌嵌出 FEEE 넌든든

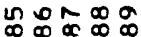
$\infty \infty \infty \infty \infty$ z Z Z Z Z 운준준준

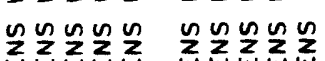

嵌亗耑亗亗出

FEE⿱E口:

$\propto \propto \alpha \propto \alpha$

$\alpha \propto \alpha \propto$

n日勺un

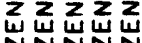
EEEE

nngen 动动品 NNNNN EEEE

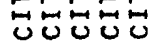

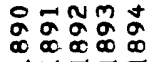

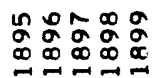

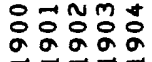
둥영영영 $2222 z$ onr $\infty \cup \infty \infty \infty$ エ岂氞志

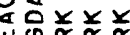

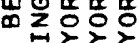
胥

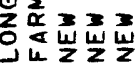

กิํํㅇ

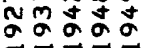
$z 2 z 2 z$ 


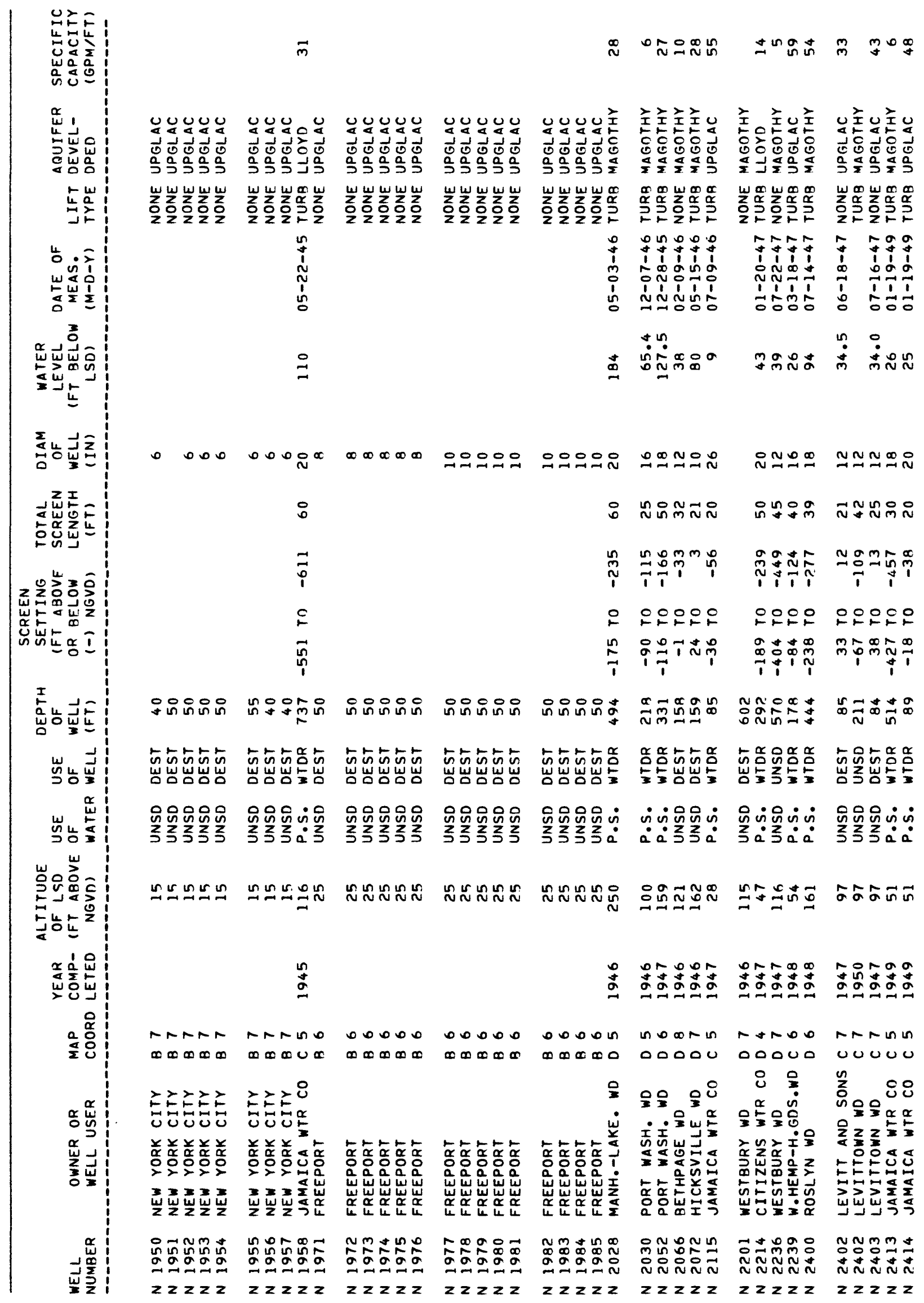




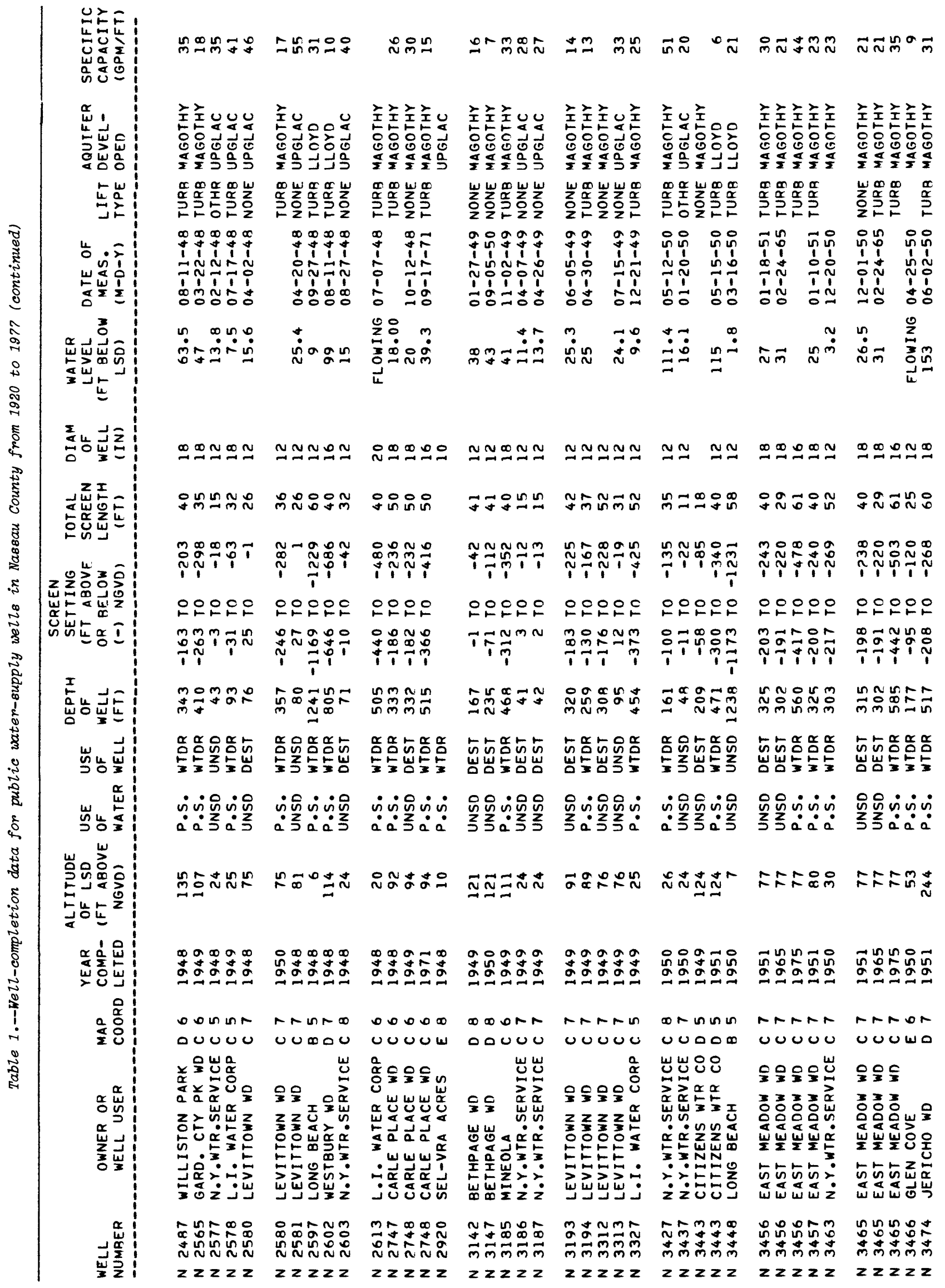




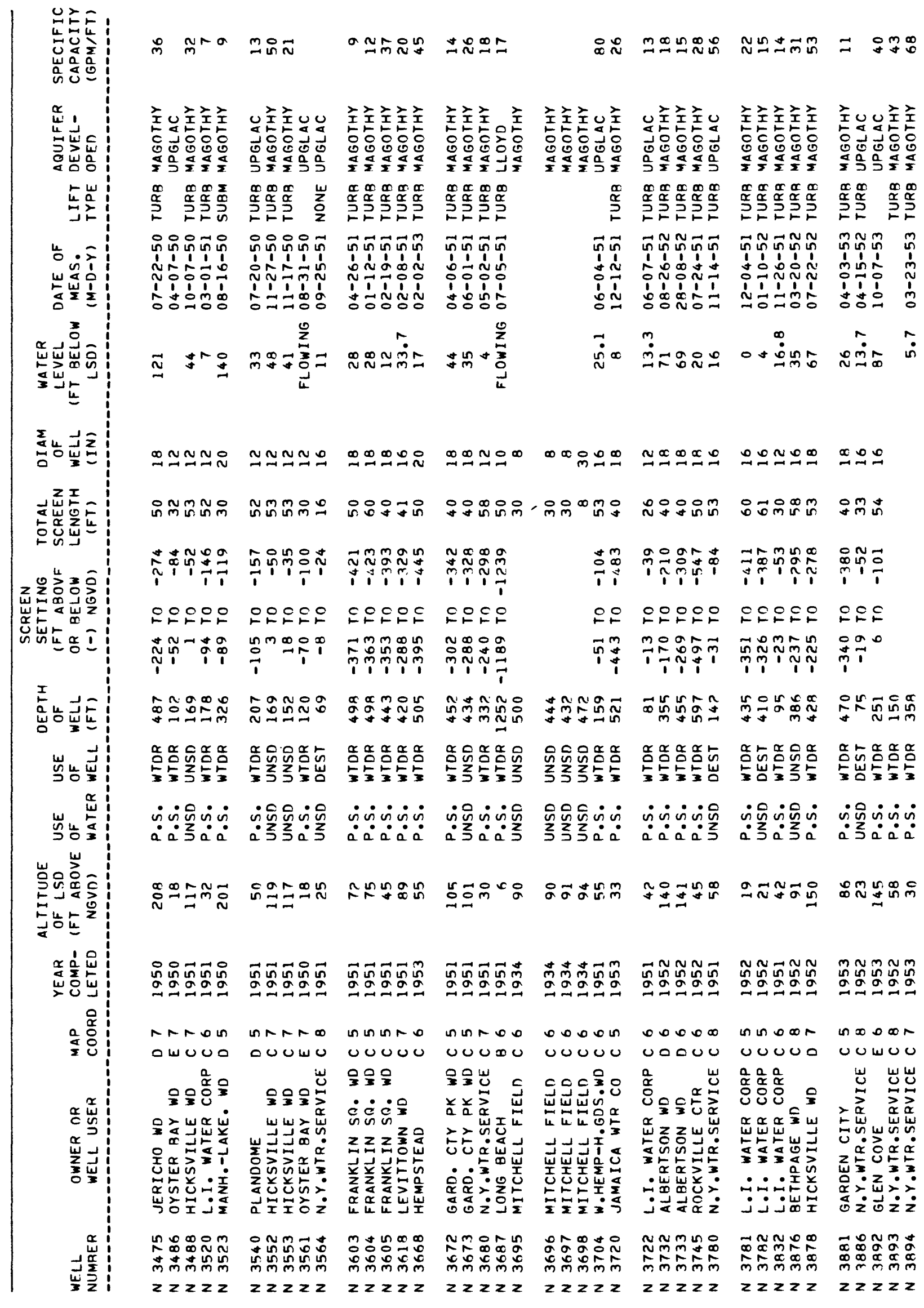




\begin{tabular}{|c|c|c|c|c|c|c|c|c|}
\hline 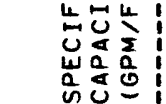 & $\pm \stackrel{\sigma}{\sim} \vec{m} \stackrel{0}{0}$ & 웧ำ & $\hat{\sim} \vec{m} \hat{m}$ & $\hat{N}=$ & 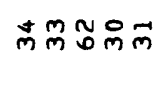 & $\stackrel{\infty}{\sim} \stackrel{\infty}{\sim}$ & $\stackrel{n}{2}$ & \\
\hline & 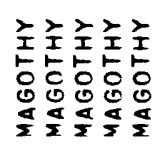 & 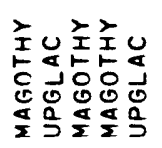 & 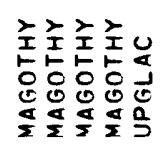 & 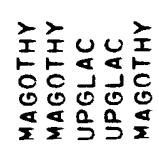 & 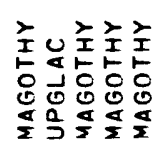 & 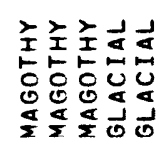 & 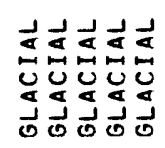 & 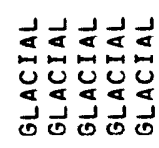 \\
\hline & 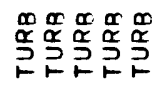 & 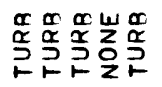 & 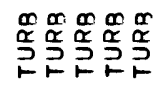 & 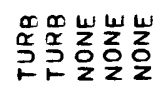 & 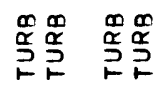 & 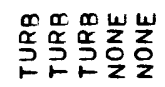 & 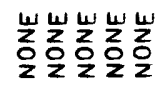 & 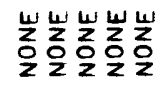 \\
\hline & 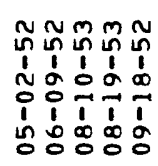 & 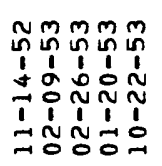 & 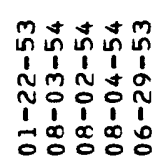 & 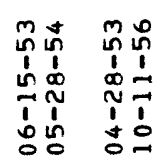 & 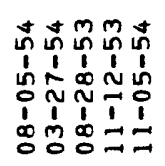 & 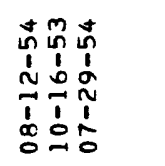 & 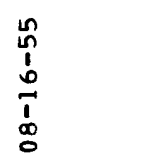 & \\
\hline & 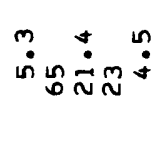 & 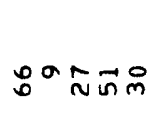 & 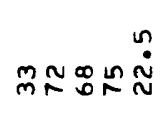 & 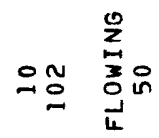 & 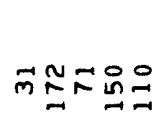 & 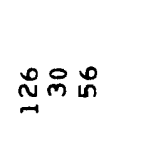 & 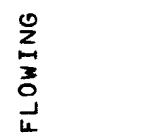 & \\
\hline & 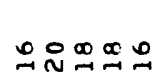 & $\stackrel{\infty}{\sim} \stackrel{0}{\sim} \underset{\sim}{\infty}$ & $\stackrel{\infty}{\sim} \underset{\sim}{\infty} \stackrel{\infty}{\sim} \stackrel{0}{\sim}$ & $\stackrel{\infty}{\simeq} \cong$ & $\stackrel{\infty}{\sim} \underset{\sim}{\infty} \stackrel{\infty}{\sim}$ & $\cong \infty$ & $\infty$ & \\
\hline & nition & బ & 은 in in in & 용 N管 & 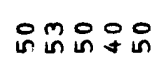 & in & $\therefore$ & \\
\hline & 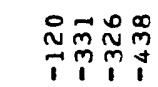 & 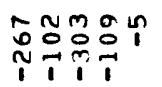 & 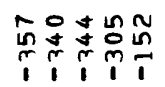 & 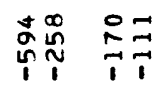 & 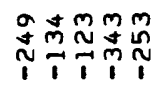 & 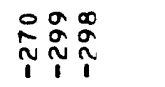 & $\vec{i}$ & \\
\hline & 안온양 & 아앙ㅇㅇ & 안야얀요 & 운요 & 은당ㅇㄴ & 암오도 & 운 & \\
\hline & 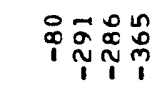 & プ & 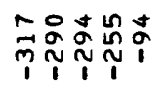 & 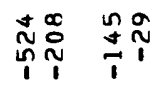 & 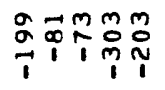 & 空苫兽 & $\vec{p}$ & \\
\hline & 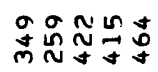 & 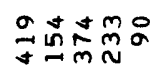 & 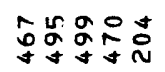 & 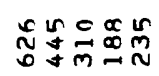 & 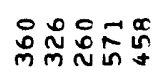 & 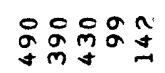 & 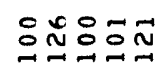 & 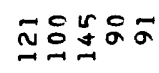 \\
\hline & 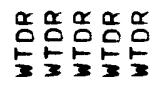 & 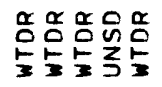 & 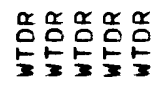 & 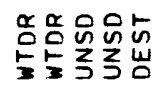 & 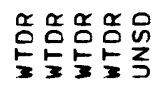 & 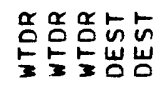 & ピ゙の゙ & 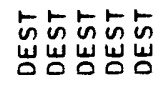 \\
\hline & 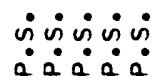 & 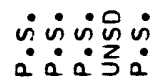 & ن் & 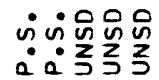 & 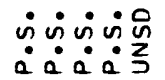 & 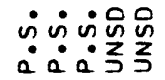 & 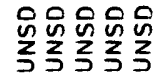 & 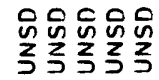 \\
\hline & 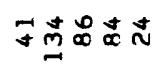 & 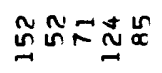 & 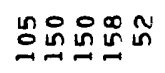 & $\stackrel{\sim}{\sim} \underset{\sim}{\sim} \underset{\sim}{\infty} \stackrel{\infty}{\sim} \underset{\sim}{ \pm}$ & 뭄 & $\stackrel{\sim}{\sim} \propto \underset{N}{\sim} \sigma a$ & 00000 & 000000 \\
\hline & 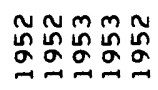 & 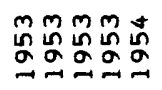 & 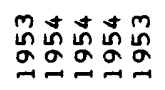 & 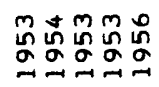 & 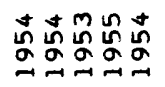 & 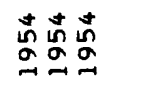 & బู & \\
\hline & 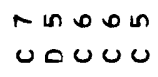 & $\begin{array}{l}n \infty \infty \infty \text { n } \\
0 \cup 000\end{array}$ & $\begin{array}{l}00000 \\
00000\end{array}$ & 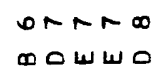 & 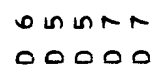 & $\begin{array}{l}\text { on } \\
\text { OUDU }\end{array}$ & $\begin{array}{l}\text { ANAN } \\
\text { UUUA }\end{array}$ & NANAN \\
\hline & 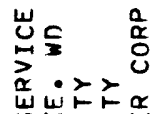 & 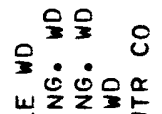 & 옹ํำ & 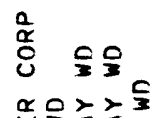 & & & 己己己こ己 & 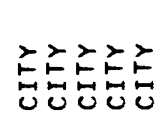 \\
\hline & 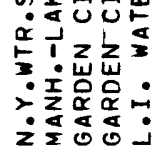 & 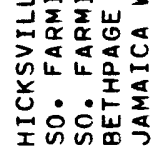 & 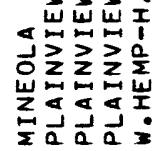 & 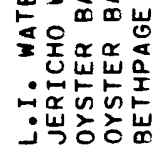 & 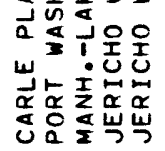 & 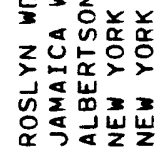 & 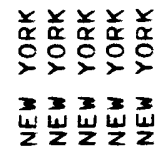 & 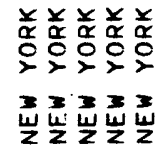 \\
\hline & 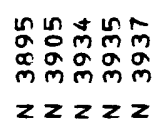 & 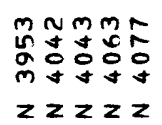 & 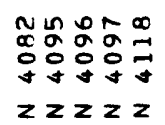 & 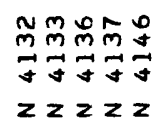 & 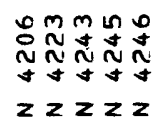 & 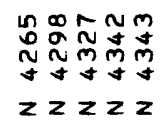 & 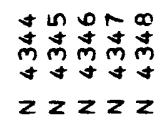 & 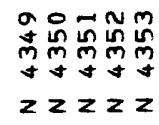 \\
\hline
\end{tabular}




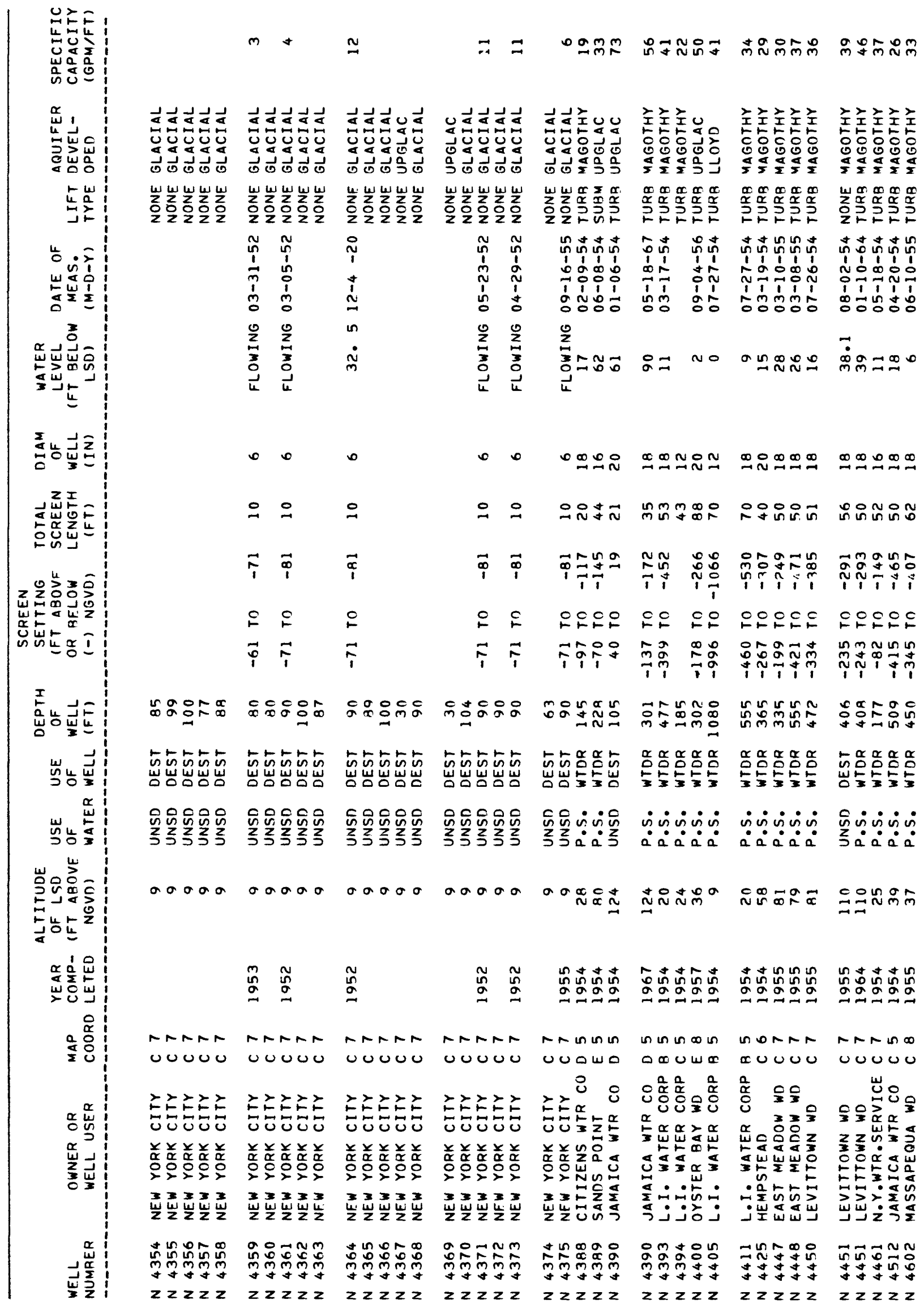




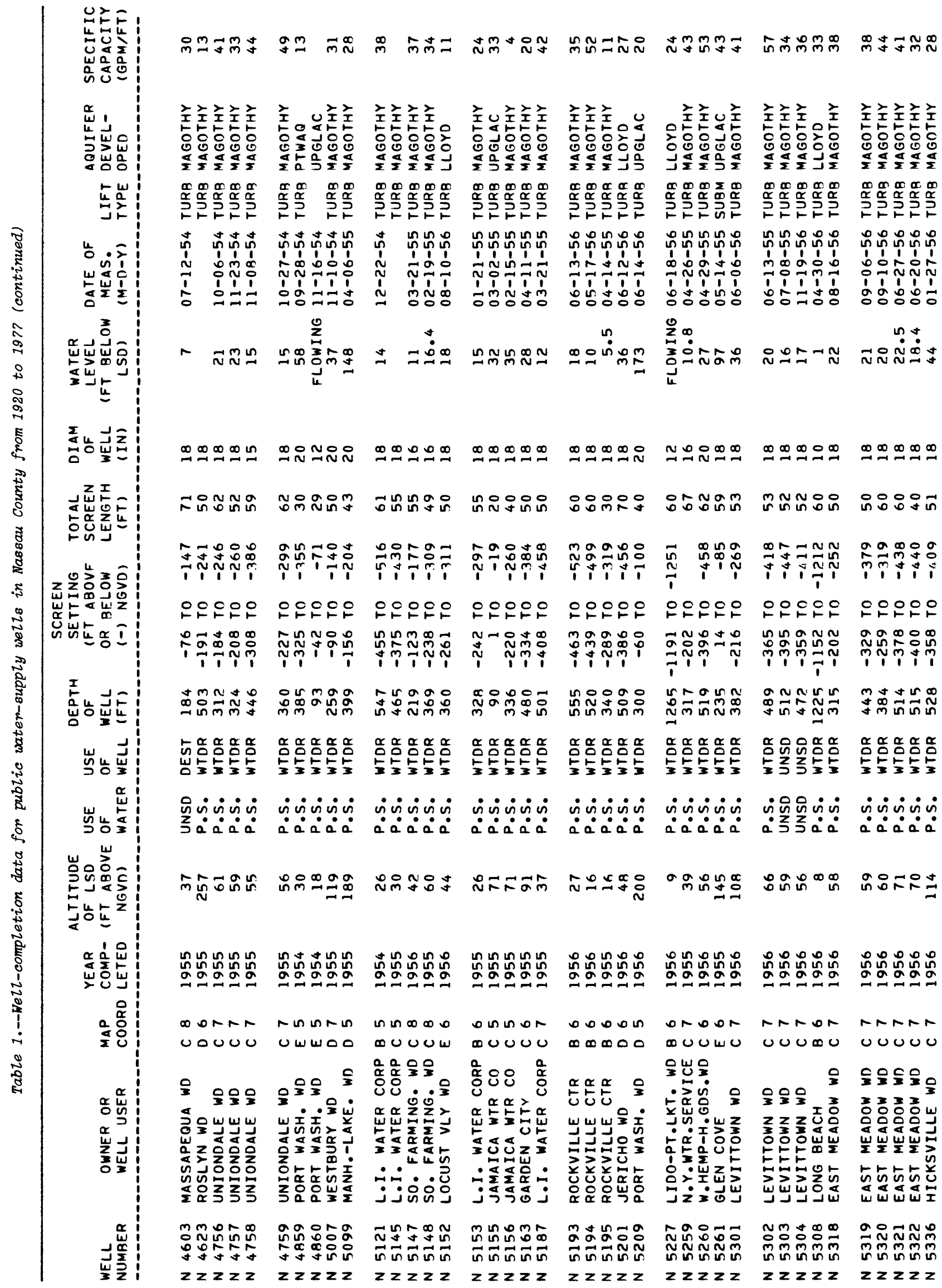




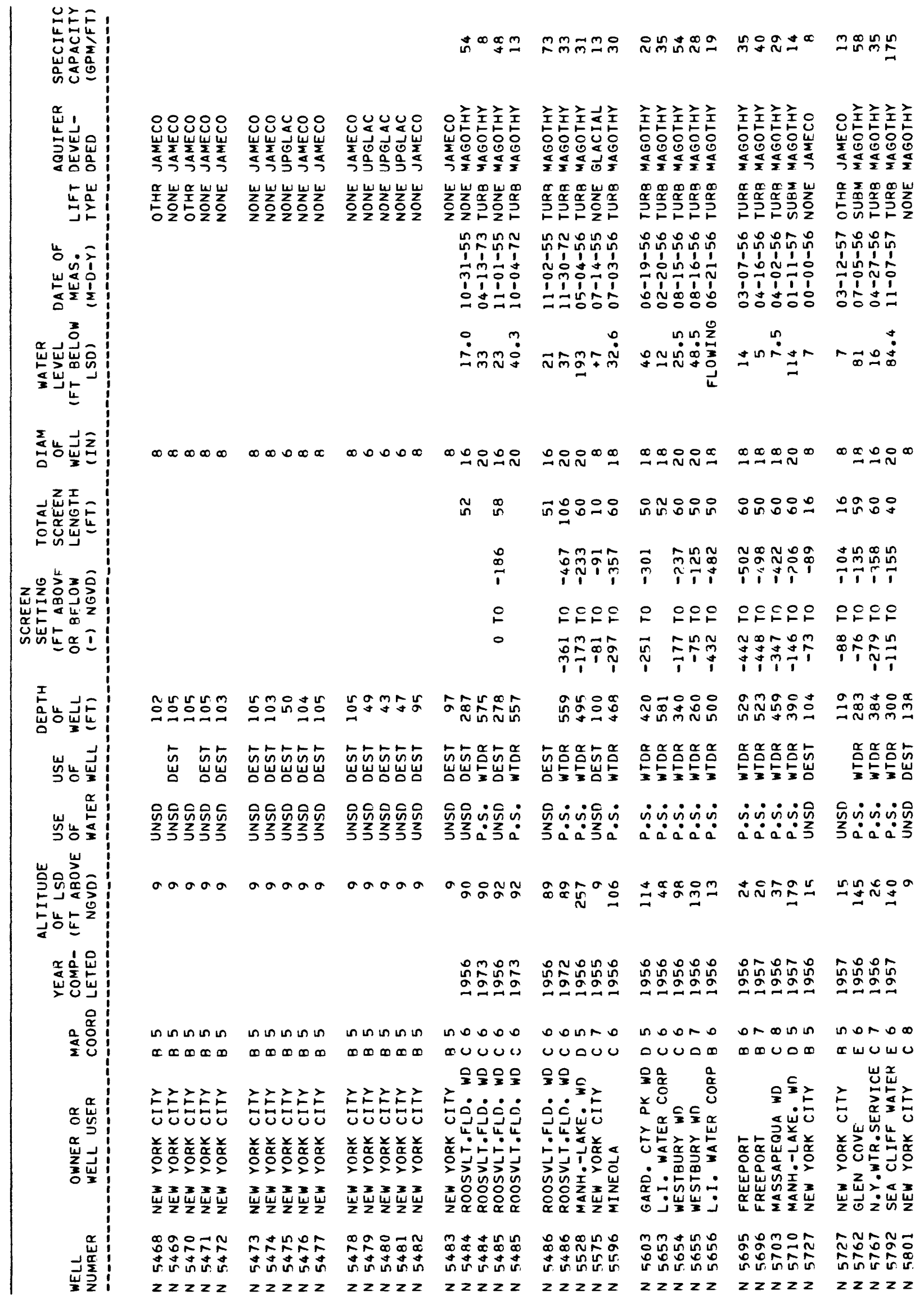




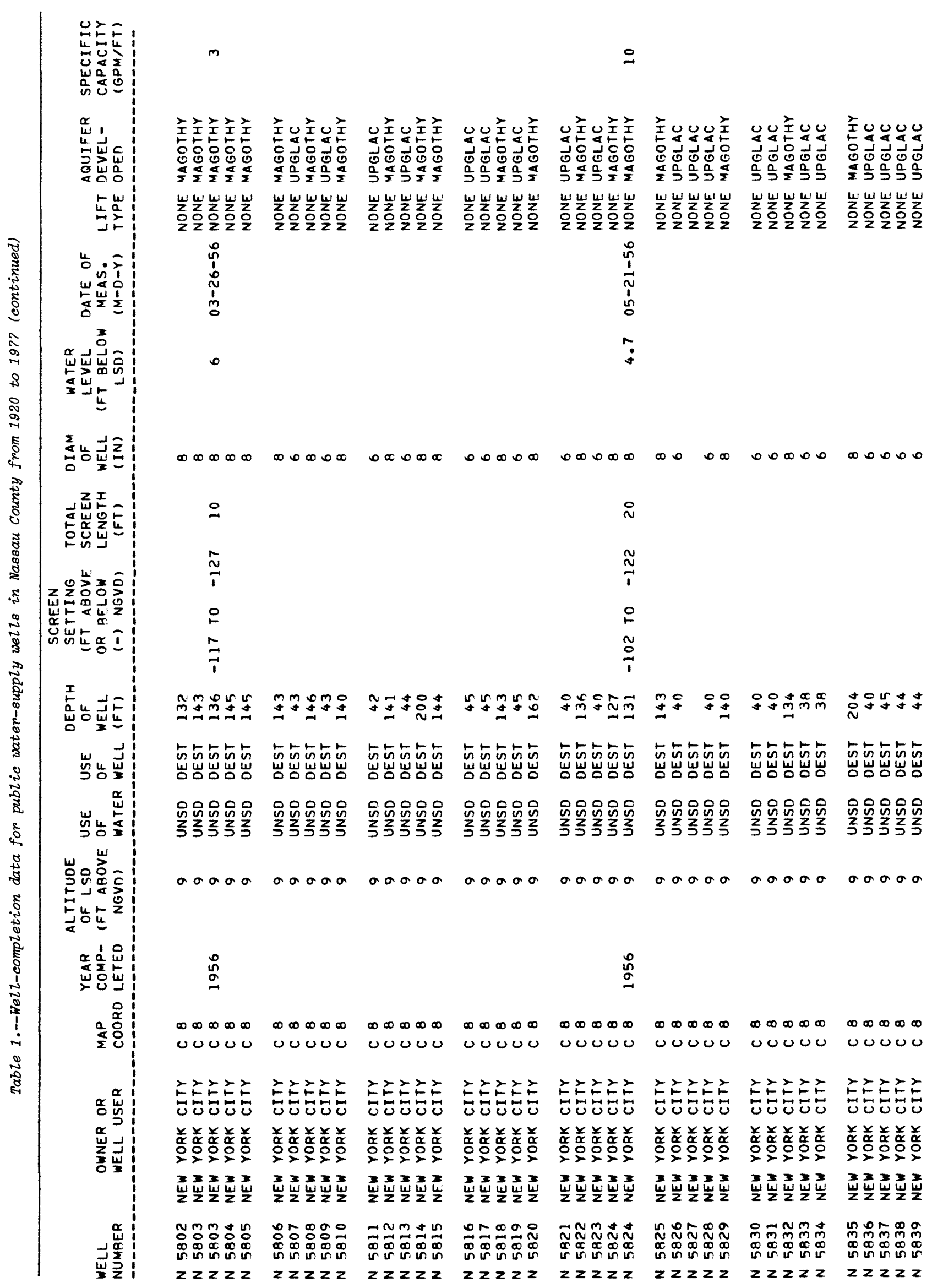




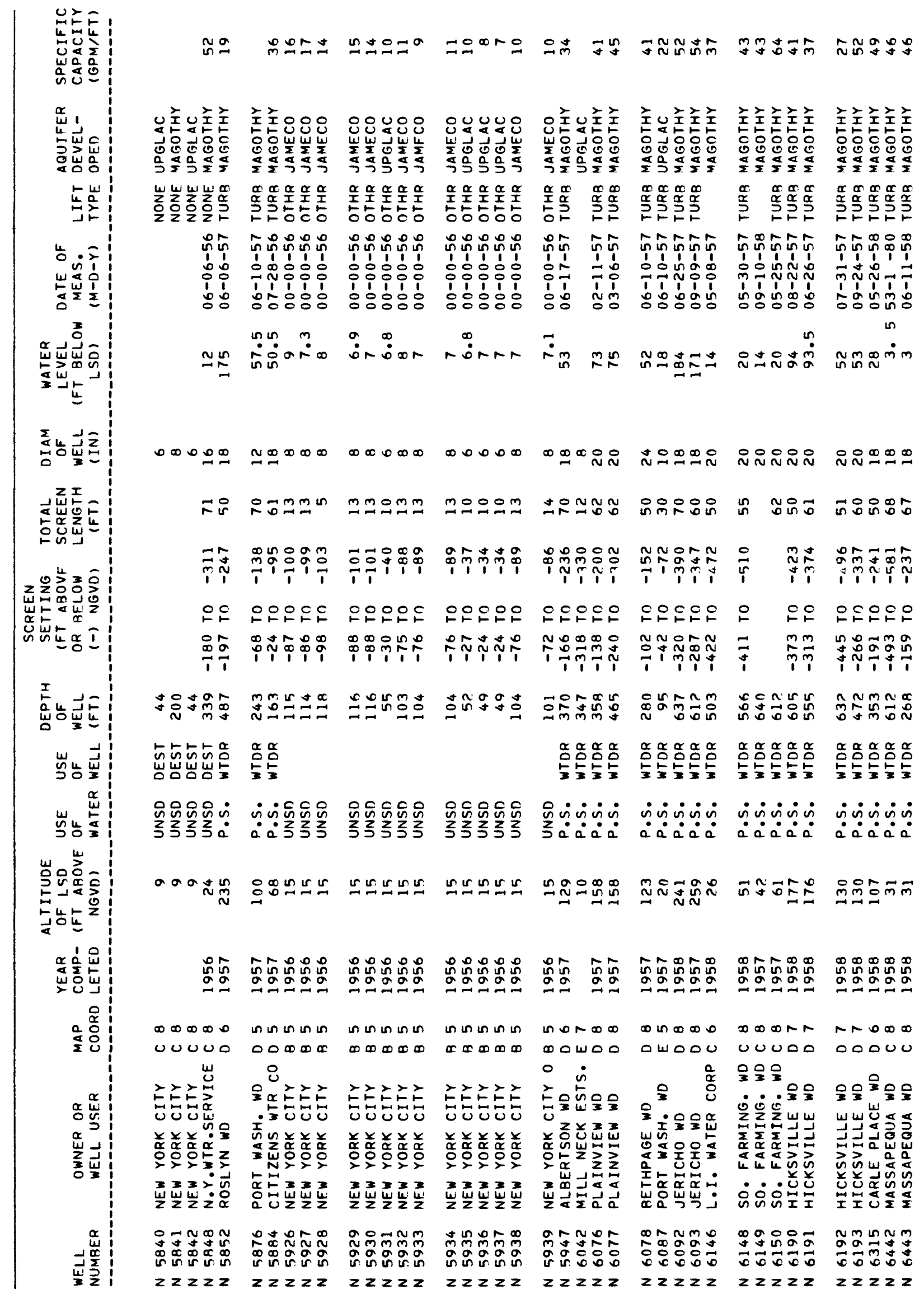




\begin{tabular}{|c|c|c|c|c|c|c|c|c|}
\hline 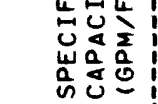 & Nก๋ & ペN品的 & กำลำก & $\exists ㅍ ㅡ N N$ & M & ㅇำㅇำ & P $\stackrel{M}{N} \stackrel{0}{N} \stackrel{0}{\sim}$ & 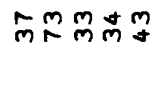 \\
\hline & 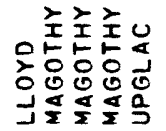 & 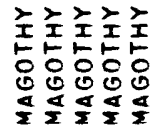 & 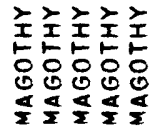 & 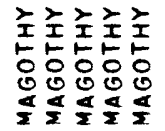 & 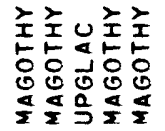 & 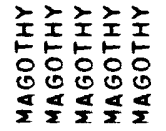 & 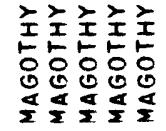 & 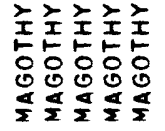 \\
\hline & 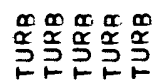 & 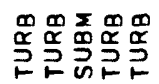 & 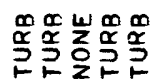 & 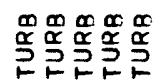 & 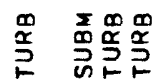 & 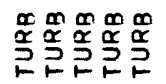 & 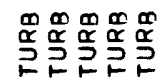 & 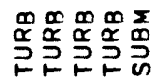 \\
\hline & 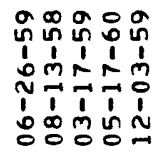 & 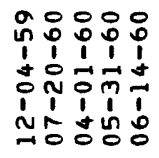 & 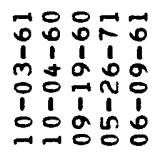 & 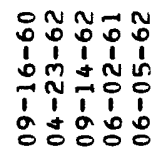 & 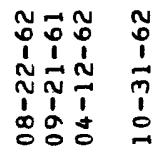 & 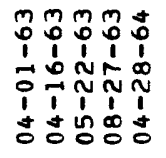 & 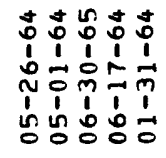 & 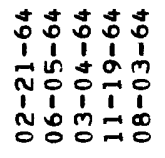 \\
\hline & น & 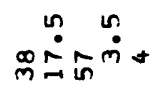 & 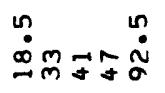 & ํำ & 苟苛 & & $\stackrel{0}{\circ} \dot{0} 0$ & $\stackrel{\sim}{0}_{\substack{m \\
\rightarrow}}$ \\
\hline & $\simeq$ & $\stackrel{\infty}{\infty} \underset{\sim}{\infty} \stackrel{\infty}{\sim} \underset{\sim}{\infty}$ & 유유요 & ํㅠํํํํำ & 윰ํำ & $\stackrel{\sim}{2} \stackrel{0}{\sim} \stackrel{\infty}{\sim}$ & ํํㄴㅇㅠ & กำก \\
\hline & 次ร早N & 的的: 0 & : in in & กำำำำ & ํำ & $\vec{F} \approx N D$ & 용요요요 & Nล้: \\
\hline & 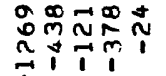 & 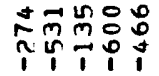 & 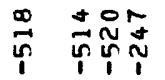 & 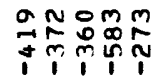 & 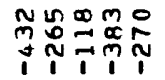 & 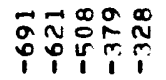 & 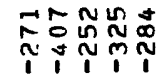 & 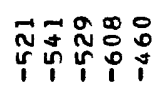 \\
\hline & 운아유요 & 움아요 & 우 운유 & 운아운요 & 오유요 & 웅유안ㅇ & Oㅜㄴㅇㅛ & 요요 \\
\hline & 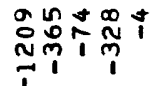 & 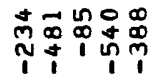 & 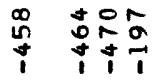 & 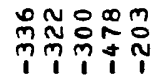 & 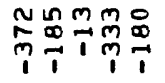 & 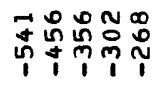 & 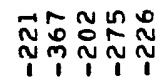 & 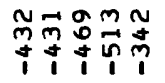 \\
\hline & 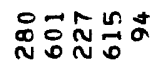 & 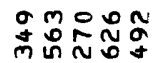 & 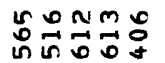 & 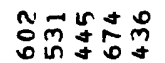 & 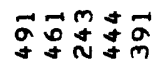 & 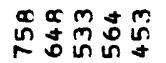 & 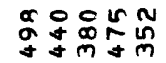 & 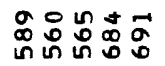 \\
\hline & 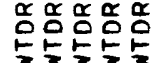 & 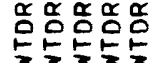 & 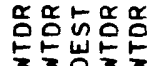 & 옹몽옹몽응 & 옹영옹뭉 & 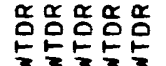 & & \\
\hline & $\dot{\varphi} \dot{\varphi} \dot{0} \dot{0}$ & 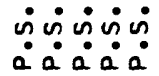 & 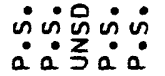 & 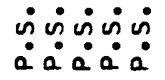 & 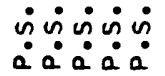 & $\dot{\varphi} \dot{\varphi} \dot{\varphi} \dot{\varphi}$ & $\dot{\varphi} \dot{\varphi} \dot{\varphi} \dot{\varphi} \dot{0}$ & $\begin{array}{l}\dot{n} \dot{\varphi} \\
\dot{a} \dot{a}\end{array}$ \\
\hline & 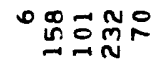 & 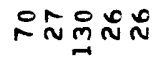 & 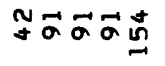 & 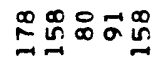 & जี & 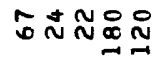 & 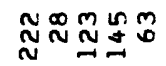 & $\stackrel{m}{0 \pm}$ \\
\hline & 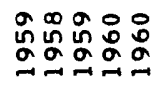 & 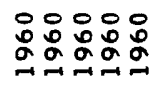 & 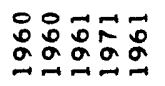 & 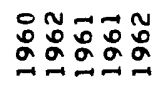 & 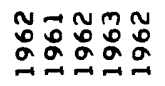 & 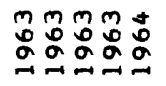 & 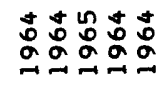 & 范范 \\
\hline & $\begin{array}{lll}n \infty & \infty\end{array}$ & & 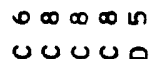 & & in in un or & & Un & \\
\hline 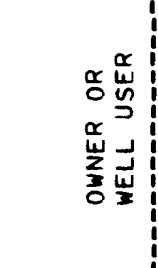 & 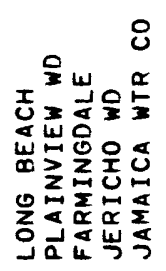 & 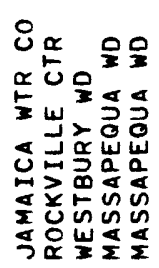 & 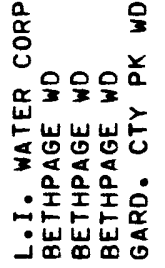 & 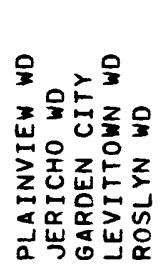 & 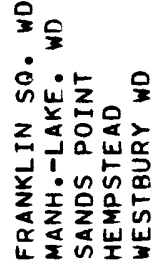 & 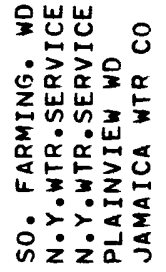 & 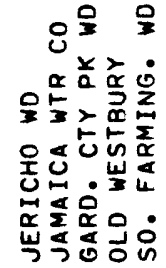 & 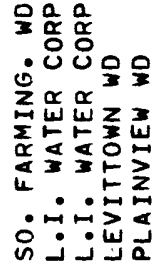 \\
\hline & 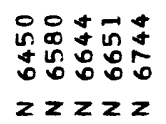 & $\begin{array}{l}n= \\
500 \\
600 \\
22\end{array}$ & 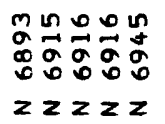 & 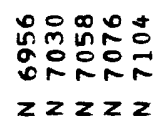 & 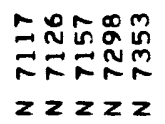 & 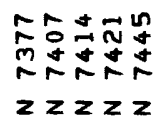 & 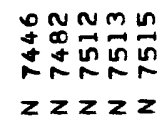 & \\
\hline
\end{tabular}




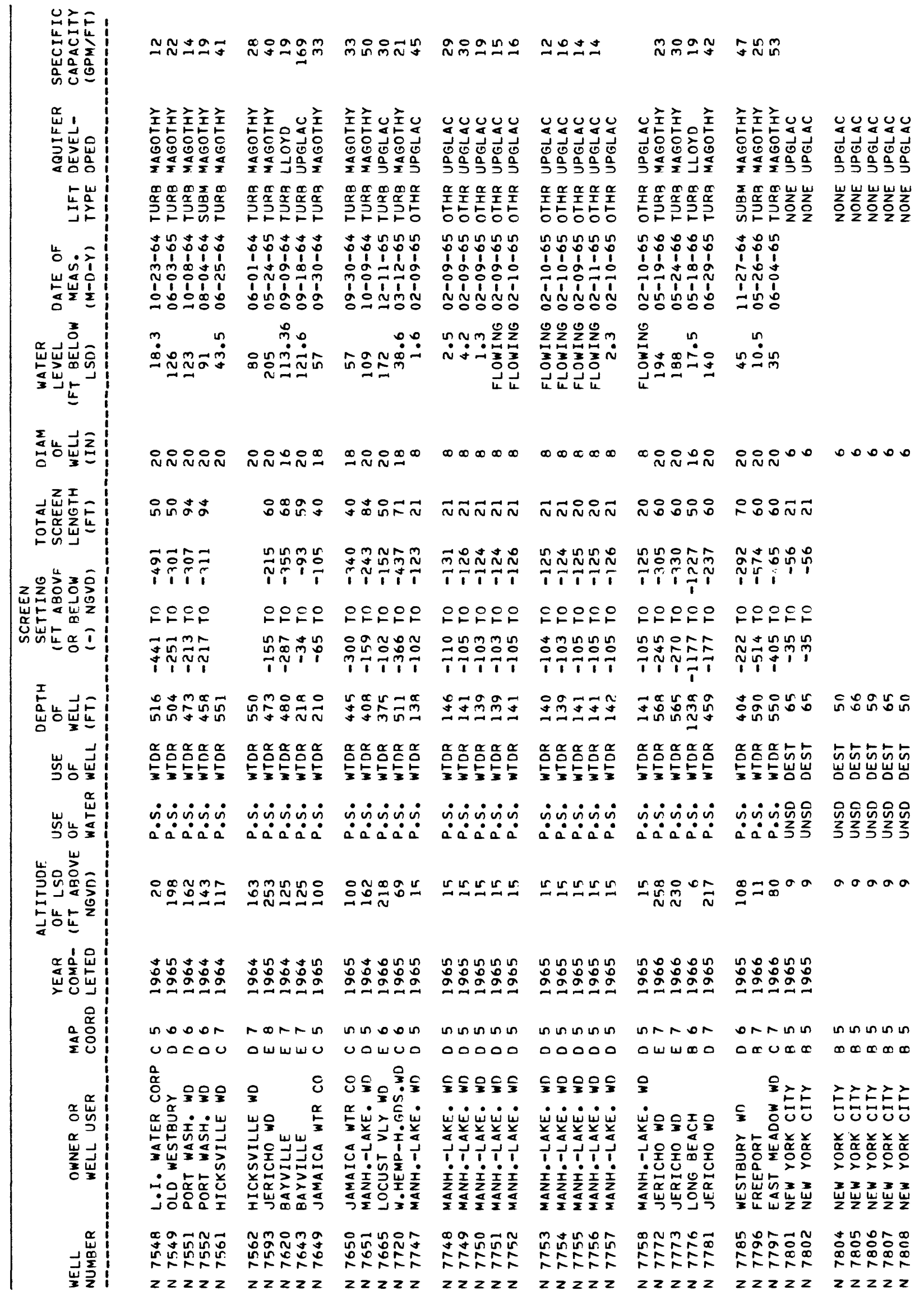




\begin{tabular}{|c|c|c|c|c|c|c|c|c|}
\hline 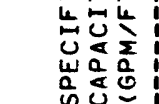 & ON $\sim R \sim N$ & 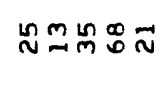 & gåñ & 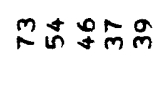 & ベホホす & $\stackrel{\infty}{N} \div \stackrel{0}{\sim} \stackrel{n}{\sim}$ & 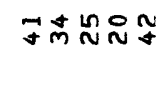 & $\stackrel{m}{m} m \tilde{N}$ \\
\hline 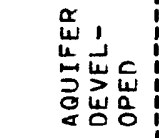 & 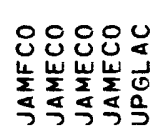 & 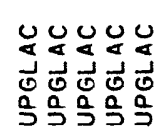 & 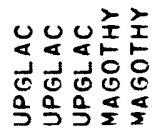 & 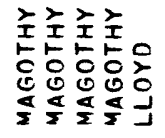 & 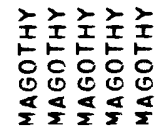 & 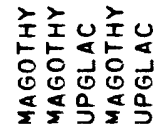 & 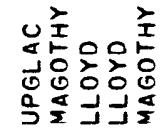 & 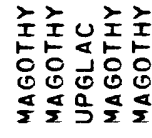 \\
\hline & 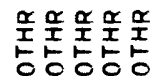 & 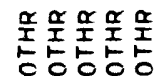 & 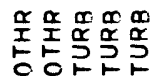 & 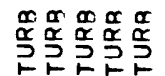 & 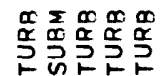 & 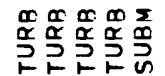 & 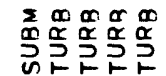 & 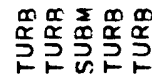 \\
\hline $\begin{array}{l}5 \\
0 \\
0 \\
0 \\
0\end{array}$ & & $\begin{array}{lll}0 & 0 & 0 \\
0 & 0 & 0 \\
1 & 1 & 1 \\
1 & 1 & 1 \\
0 & 0 & 0 \\
1 & 1 & 1 \\
\sigma & \alpha & \alpha \\
0 & 0 & 0\end{array}$ & 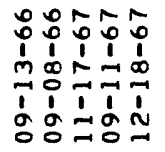 & 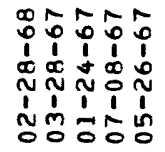 & 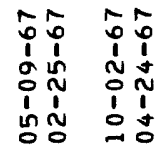 & 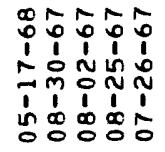 & 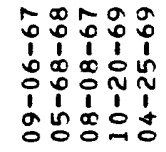 & 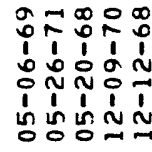 \\
\hline & $\infty \infty \infty \infty \infty$ & $\infty \infty \infty \infty \infty$ & $\infty \infty$ 况 & ํํํㅇํำ & ํㅜํ유유 & 유ํํํำ & 옹ํํ요 & 䠌 ${ }^{\infty}{ }_{N}$ \\
\hline & 오ํํํํำ & 음ㅇㅇㅇ & 웅ㅇㅇㅇㅇㅇ & x: $:$ in & $\stackrel{n}{\infty}: 0: 0: 0$ & in $\vec{F} \stackrel{\infty}{\infty}$ in & 品品 $\overrightarrow{6}$ 织只 & 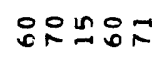 \\
\hline & 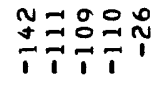 & $\stackrel{\sim}{\sim} \underset{\sim}{\sim} \underset{\sim}{\sim} \underset{\sim}{\sim} \underset{\sim}{\sim}$ & ثิن & 足总 & 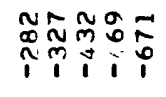 & 角 & 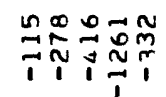 & 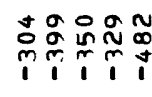 \\
\hline & 운요 & EFEEO & OCECE & 안앙ㅇㄴ & 단안안 & 암요 & 은ㄷㅇㄴ & OOORE \\
\hline & 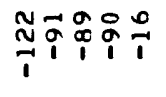 & 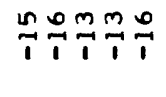 & 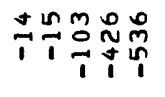 & 点足足 & 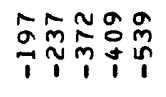 & $\begin{array}{l}0 \\
0 \\
0\end{array}$ & 1̊ & 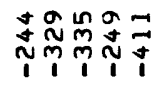 \\
\hline & 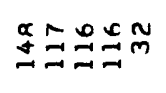 & 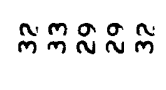 & 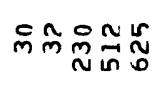 & 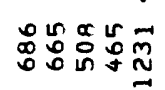 & 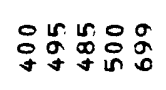 & 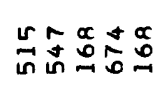 & 象商志占占 & 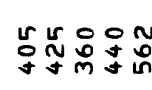 \\
\hline & 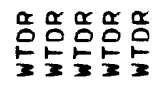 & 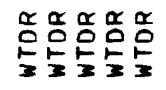 & 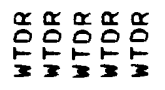 & 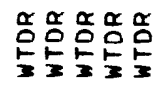 & 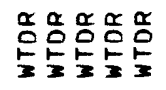 & 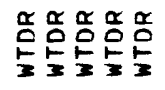 & 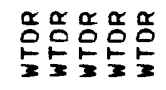 & 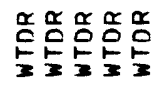 \\
\hline & ம்ம்ம் & $\dot{\varphi} \dot{\varphi} \dot{\varphi} \dot{\varphi} \dot{0}$ & $\dot{\varphi} \dot{\varphi} \dot{\varphi} \dot{\varphi} \dot{a}$ & 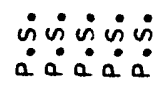 & $\dot{\varphi} \dot{\varphi} \dot{\varphi} \dot{\varphi} \dot{0}$ & 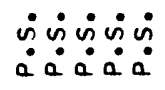 & 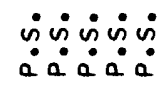 & $\begin{array}{l}\dot{n} \dot{\varphi} \dot{\varphi} \\
\dot{a} \dot{a}\end{array}$ \\
\hline 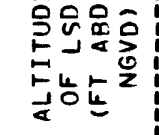 & in in in $\ln$ is & in so in in 5 & $\ln \propto \underset{\sim}{\sim} \tilde{N}$ & 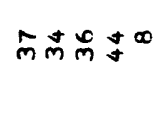 & 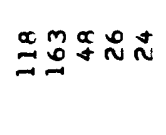 & 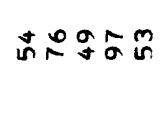 & in $\infty \stackrel{\infty}{\sim} \underset{\sim}{\stackrel{\infty}{N}}$ & ஃே゙゚ロ \\
\hline 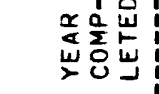 & 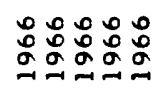 & : & 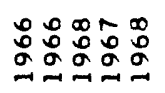 & 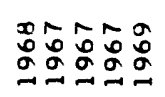 & 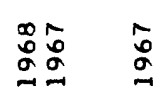 & 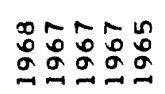 & 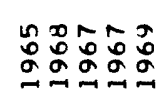 & 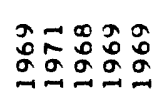 \\
\hline & 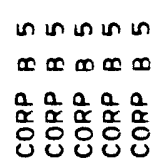 & 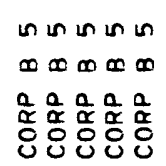 & 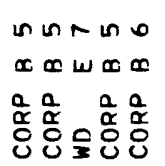 & $\begin{array}{l}\infty 0000 \\
00000 \\
0.000\end{array}$ & 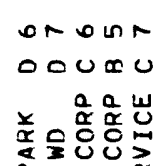 & 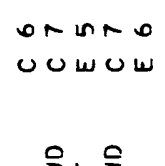 & 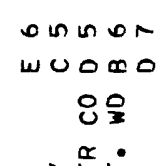 & 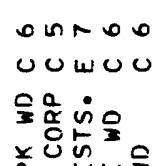 \\
\hline & 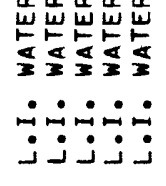 & ذّ & 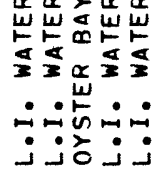 & 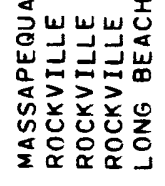 & 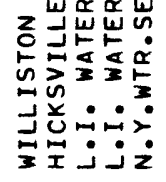 & 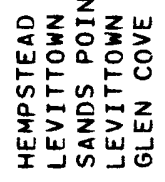 & 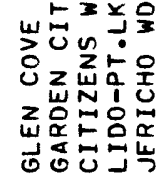 & 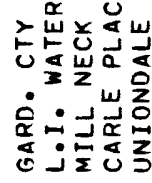 \\
\hline سִ & 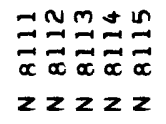 & 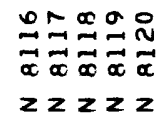 & 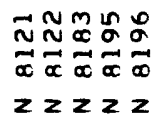 & $\begin{array}{l}\underset{\sim}{\sim} \underset{\alpha}{\mathbb{N}} \underset{\sim}{N} \underset{\sim}{N} \underset{\sim}{N} \\
z z z z z\end{array}$ & 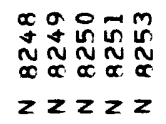 & 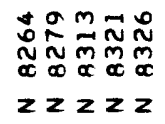 & 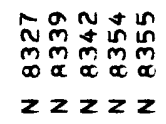 & 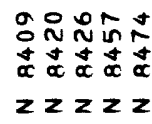 \\
\hline
\end{tabular}




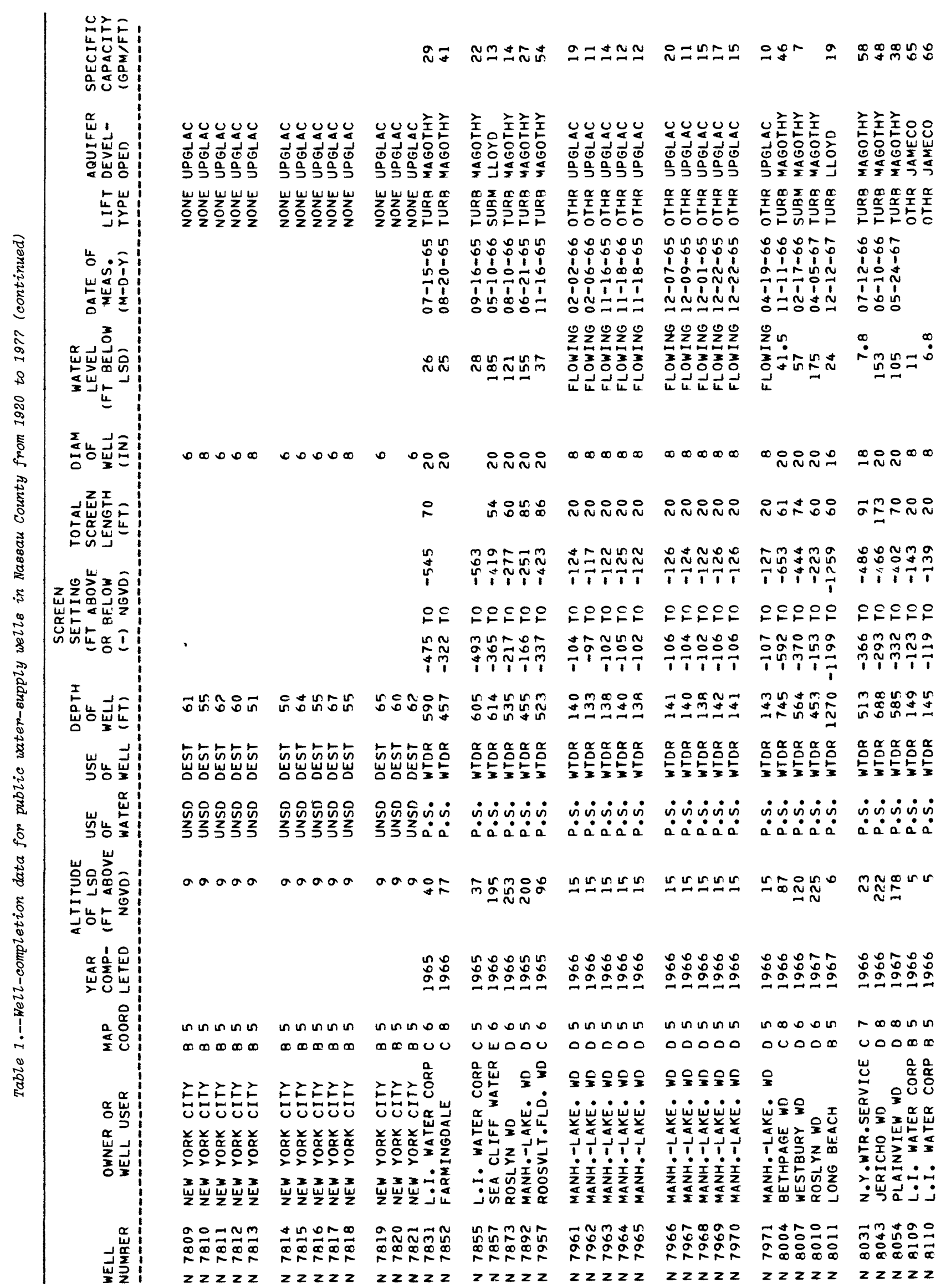




\begin{tabular}{|c|c|c|c|c|c|c|c|c|}
\hline 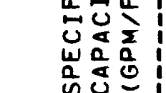 & 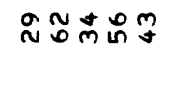 & 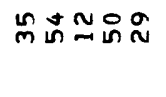 & $\stackrel{m}{N} g=g r$ & ถัำฏ & 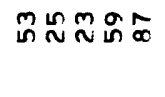 & $\hat{m} \mathscr{g} \bar{m} \tilde{n} \tilde{m}$ & $\stackrel{\infty}{*} \mathfrak{R} \tilde{n} \pm$ & ヘテュッさ \\
\hline 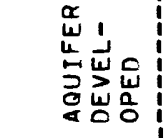 & 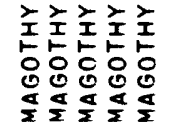 & 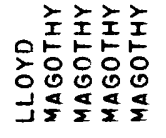 & 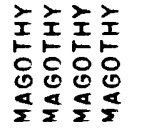 & 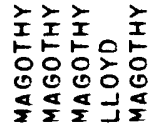 & 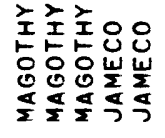 & 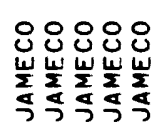 & 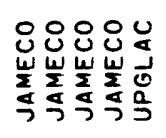 & 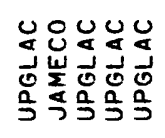 \\
\hline & 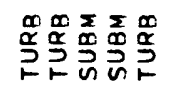 & 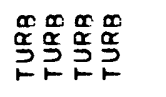 & 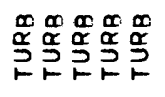 & 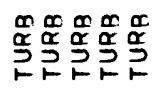 & 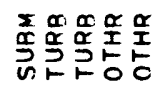 & 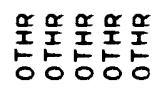 & 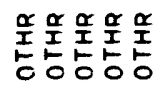 & 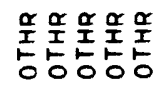 \\
\hline " & 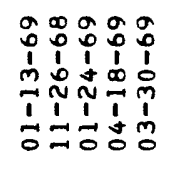 & 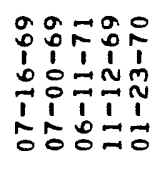 & 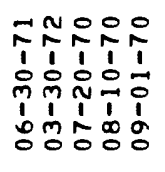 & 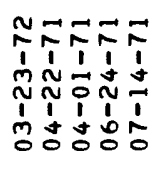 & 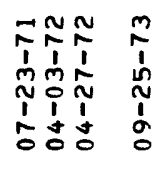 & 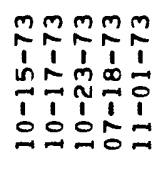 & 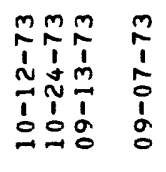 & 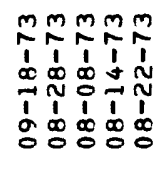 \\
\hline & 紊芯 & 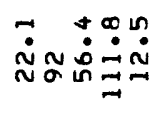 & 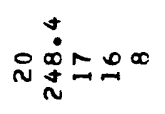 & 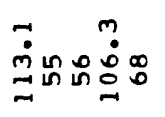 & & 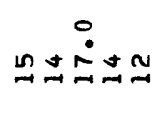 & $\cong \cong \pm$ & $0 \cong \simeq$ \\
\hline & ํㅠㅇㅛ & ํํํํํำ & $\infty$ 옹ำ & ำㅇำ & 웃ㅇㅇㅇ & $\infty \infty \infty$ & $\infty \infty \infty \propto \infty$ & $\infty \infty \infty \infty$ \\
\hline & 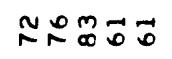 & in $: 0: 0 \overline{0}$ & :ํำโกิ & $: \overrightarrow{0} \overrightarrow{0}: \overrightarrow{0}$ & ํํำกำ & 오오ํำ & ํํํ요으 & 융ㅇㅇ응 \\
\hline & 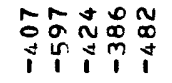 & 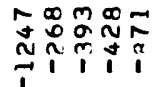 & 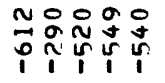 & $\begin{array}{l}+0 \\
0 \\
\\
1 \\
1\end{array}$ & 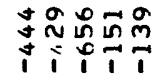 & 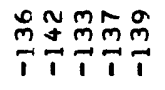 & 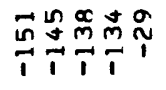 & 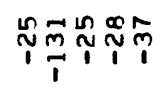 \\
\hline & OOERO & 암요요 & 든요 & 은용 & 안아요 & 요요 & 앙ㅇㅁ앙ㅇㅇㅇ & 운다요 \\
\hline & 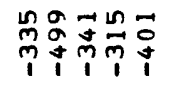 & 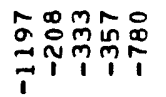 & 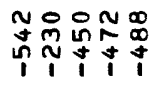 & 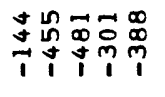 & 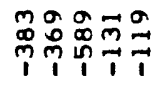 & 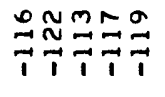 & 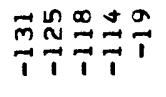 & 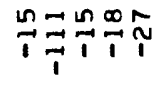 \\
\hline & 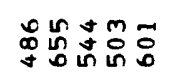 & 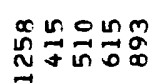 & 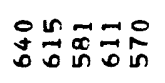 & 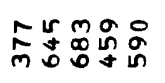 & 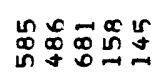 & 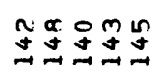 & 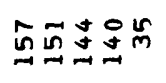 & 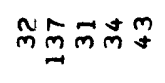 \\
\hline & 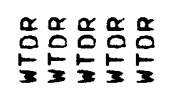 & 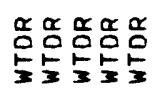 & 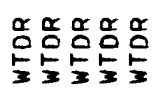 & 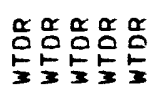 & 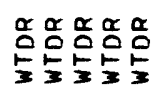 & 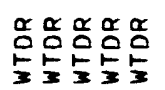 & 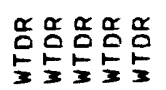 & 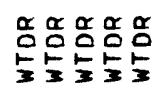 \\
\hline & $\dot{\varphi} \dot{\varphi} \dot{\varphi} \dot{\varphi}$ & $\dot{\varphi} \dot{\varphi} \dot{\varphi} \dot{\varphi}$ & $\dot{\varphi} \dot{\varphi} \dot{\varphi} \dot{\varphi}$ & $\begin{array}{l}\dot{n} \dot{n} \dot{n} \dot{n} \\
\dot{a} a \dot{a}\end{array}$ & $\begin{array}{l}\dot{n} \dot{\varphi} \dot{\varphi} \dot{0} \\
\dot{a} a \dot{a}\end{array}$ & $\dot{\varphi} \dot{\varphi} \dot{a} \dot{a} \dot{a}$ & $\begin{array}{l}\dot{\varphi} \dot{\varphi} \dot{\varphi} \\
\dot{a} \dot{a}\end{array}$ & 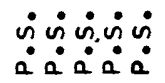 \\
\hline & ホ怘怘ニす & $0 \underset{ \pm \sim}{\sim \sim N N}$ & 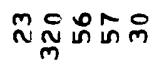 & 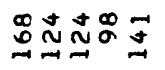 & 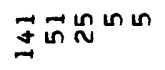 & in $\ln$ & $\ln n \pi$ & \\
\hline & 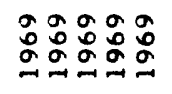 & 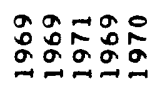 & 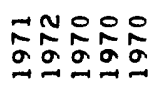 & NลテNล & 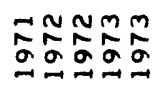 & 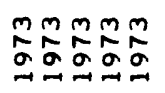 & 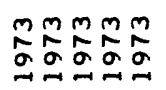 & Mำ? \\
\hline & 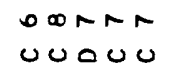 & 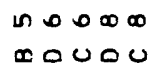 & $\begin{array}{l}\text { ORDOR } \\
\text { couUu }\end{array}$ & ODONR & $\begin{array}{l}\text { N } \ln \infty \text { in in } \\
0 \cup \cup \infty \infty\end{array}$ & 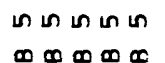 & 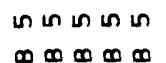 & 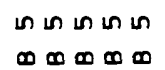 \\
\hline & 岂 & & 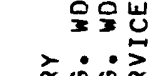 & & & 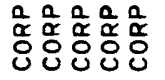 & 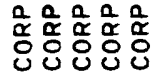 & 年 \\
\hline & 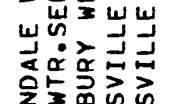 & 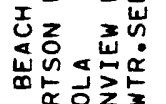 & 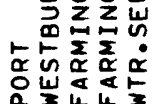 & 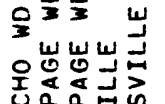 & 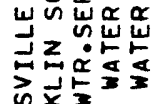 & 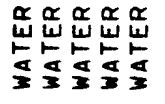 & 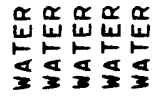 & $\begin{array}{l}0 \\
0\end{array}$ \\
\hline & 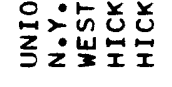 & 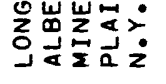 & 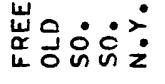 & 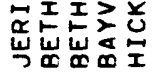 & 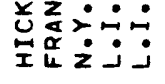 & ذّنّ & & \\
\hline$\frac{\alpha}{u}$ & 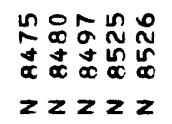 & 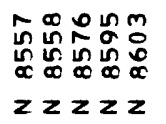 & 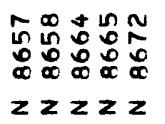 & 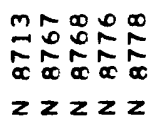 & 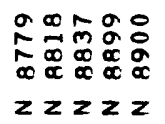 & 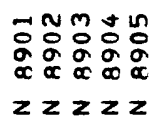 & 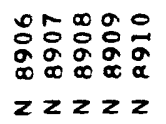 & $\begin{array}{l}-7 \pi \\
5 \% \\
0 \% \\
22\end{array}$ \\
\hline
\end{tabular}









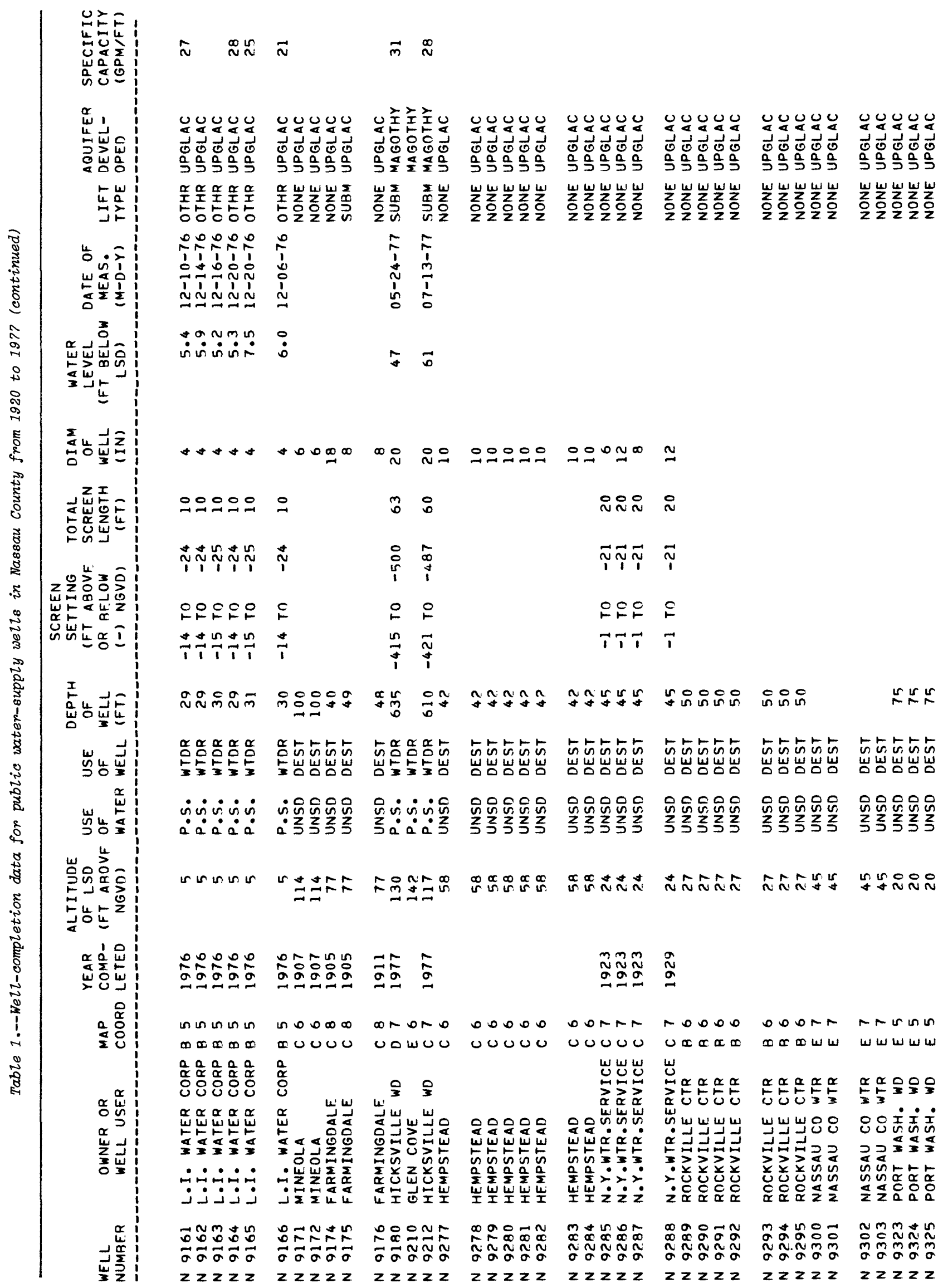




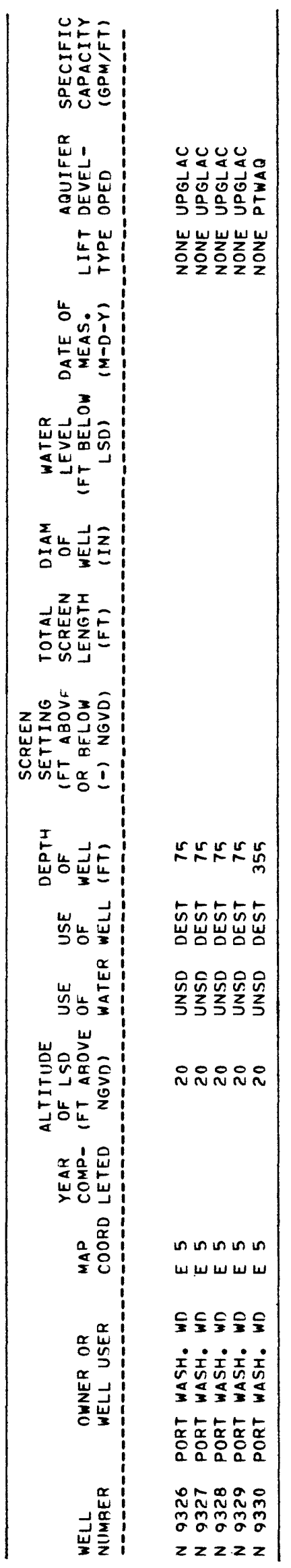




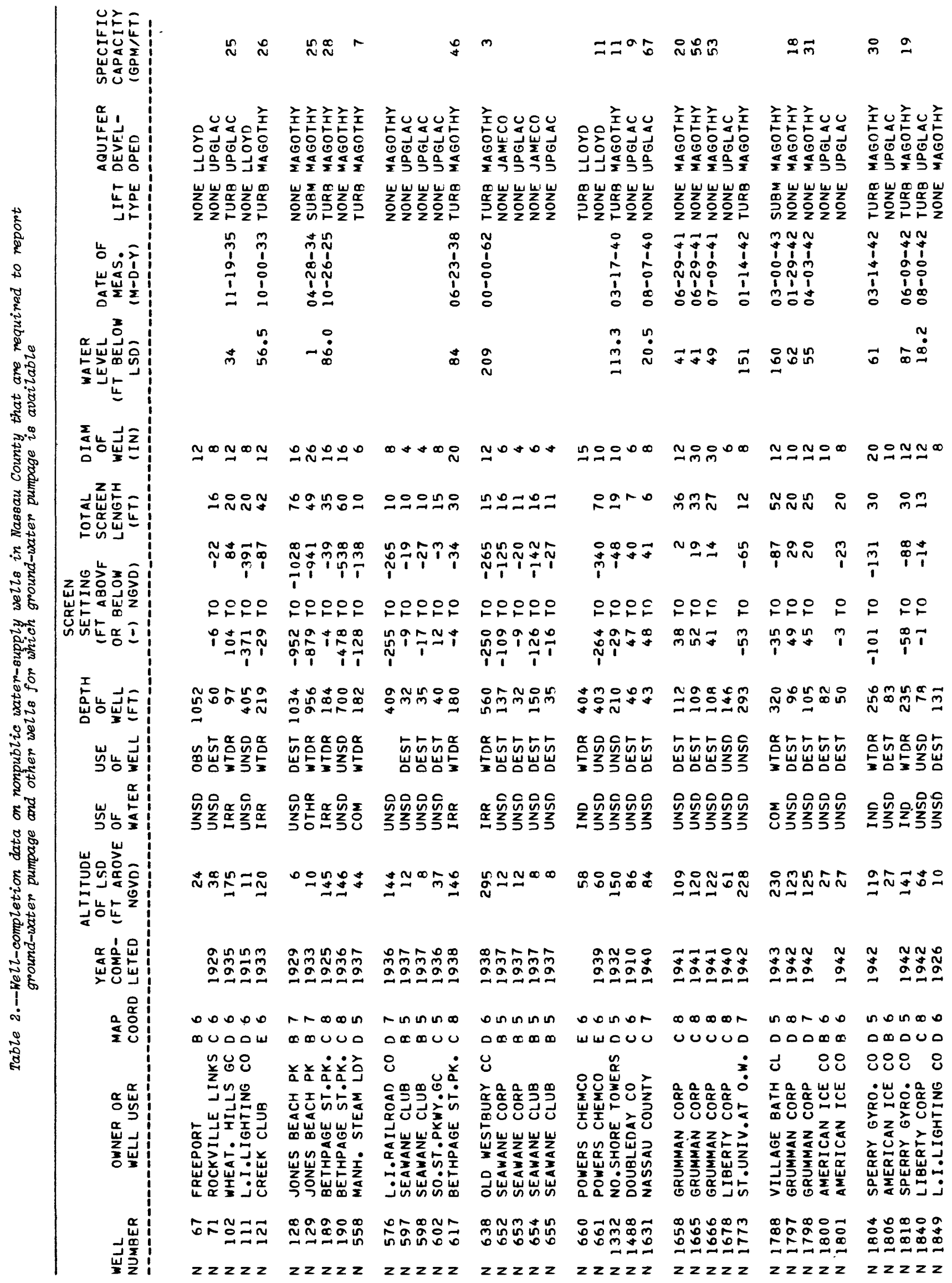




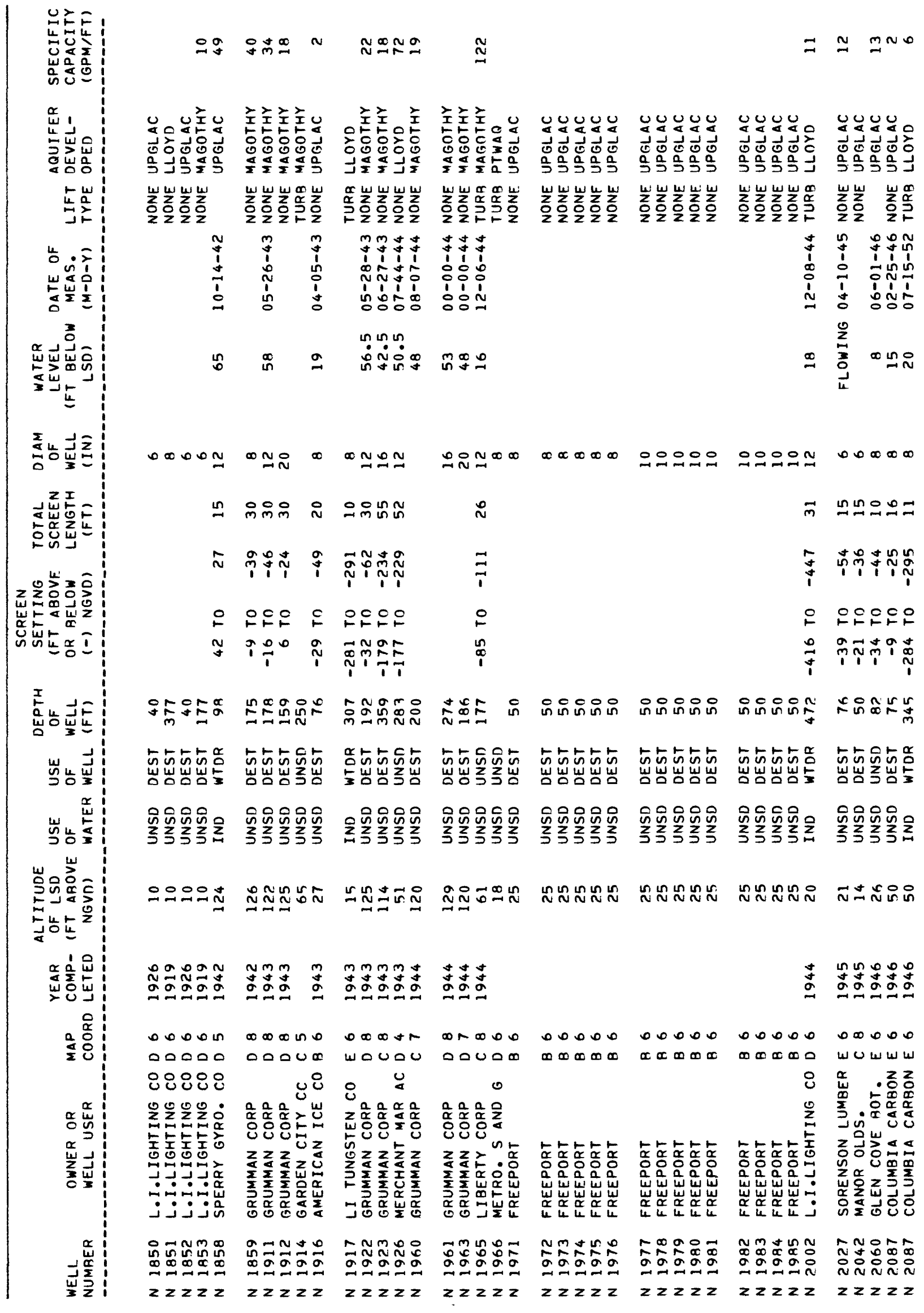




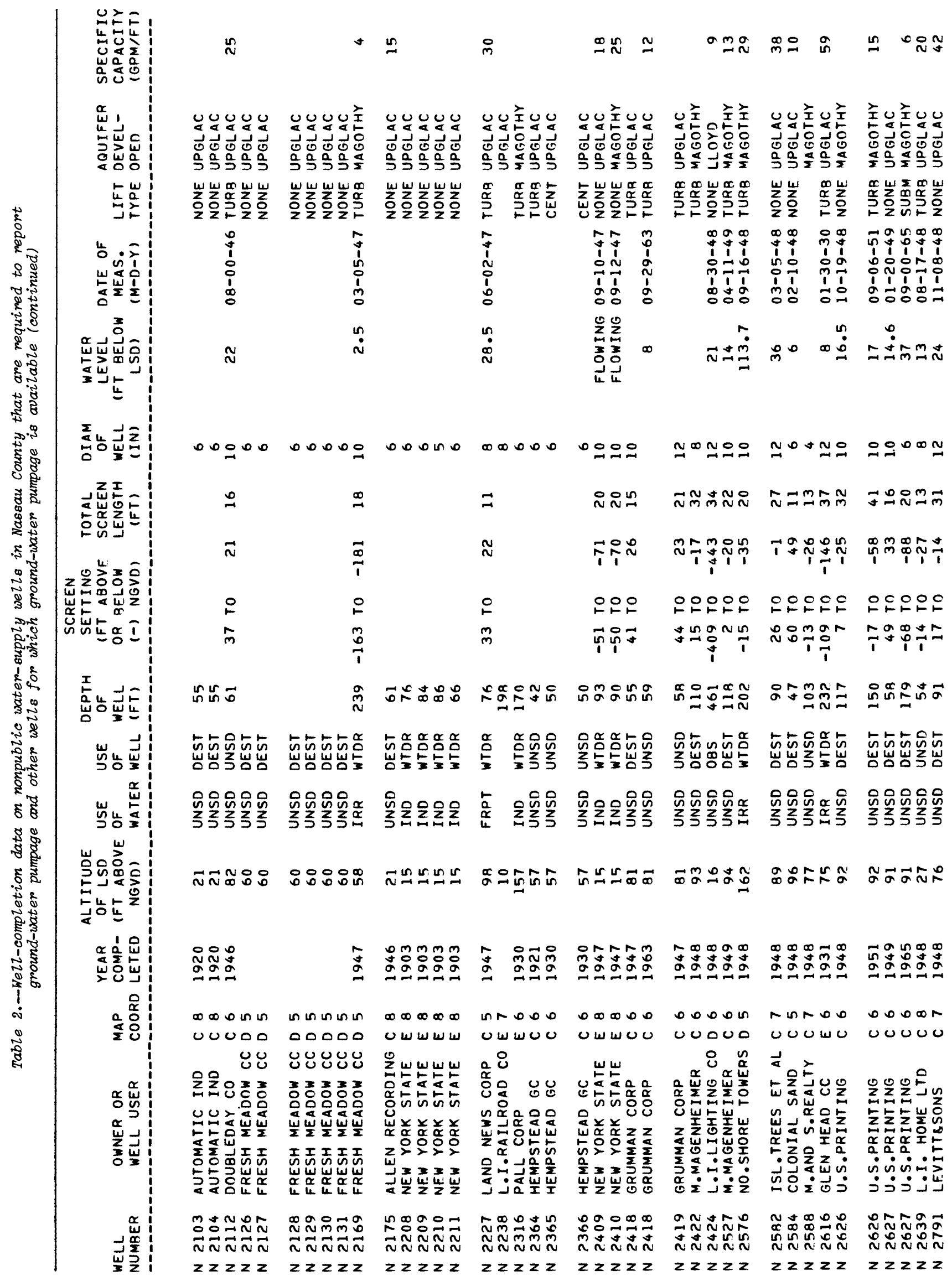




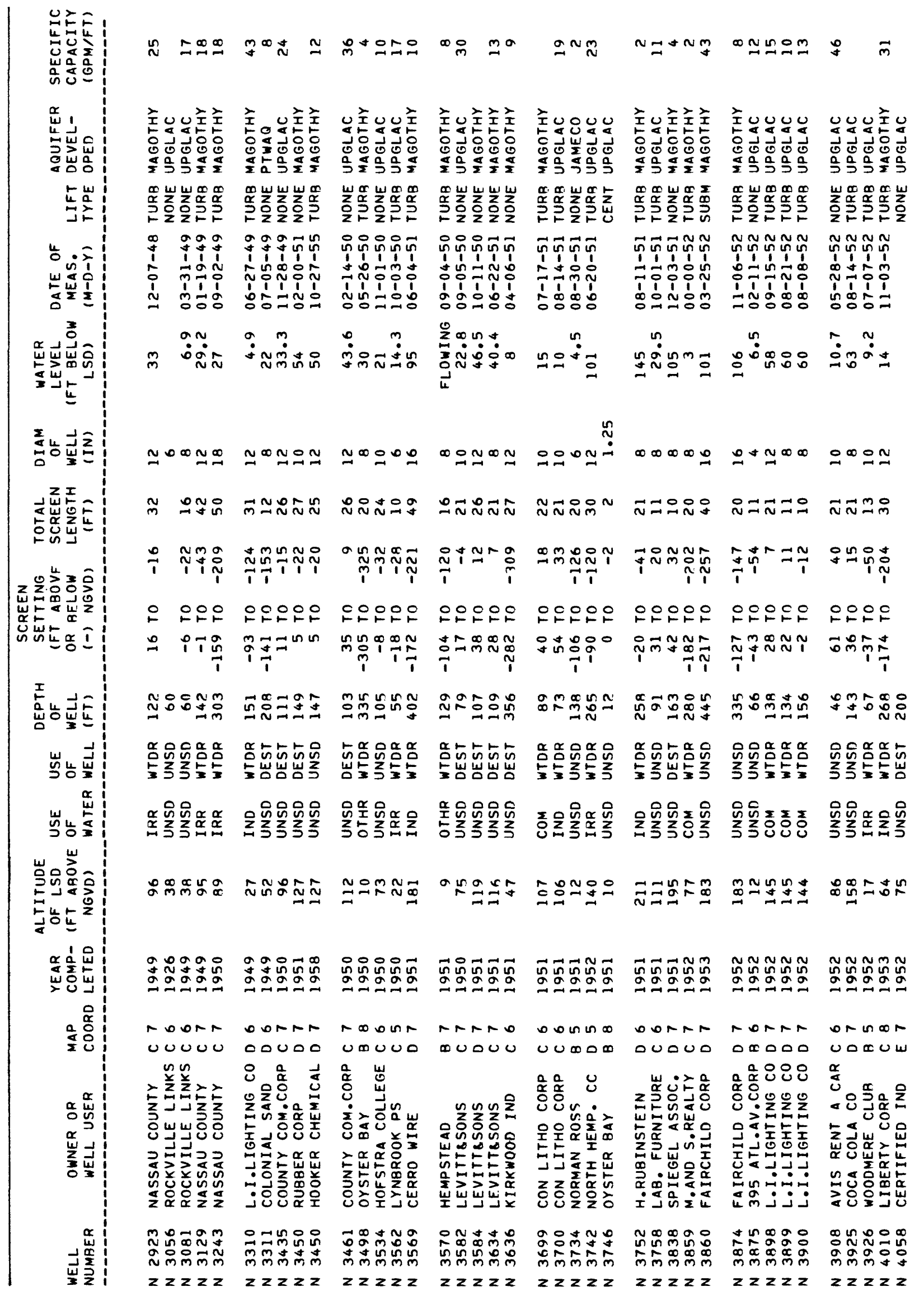




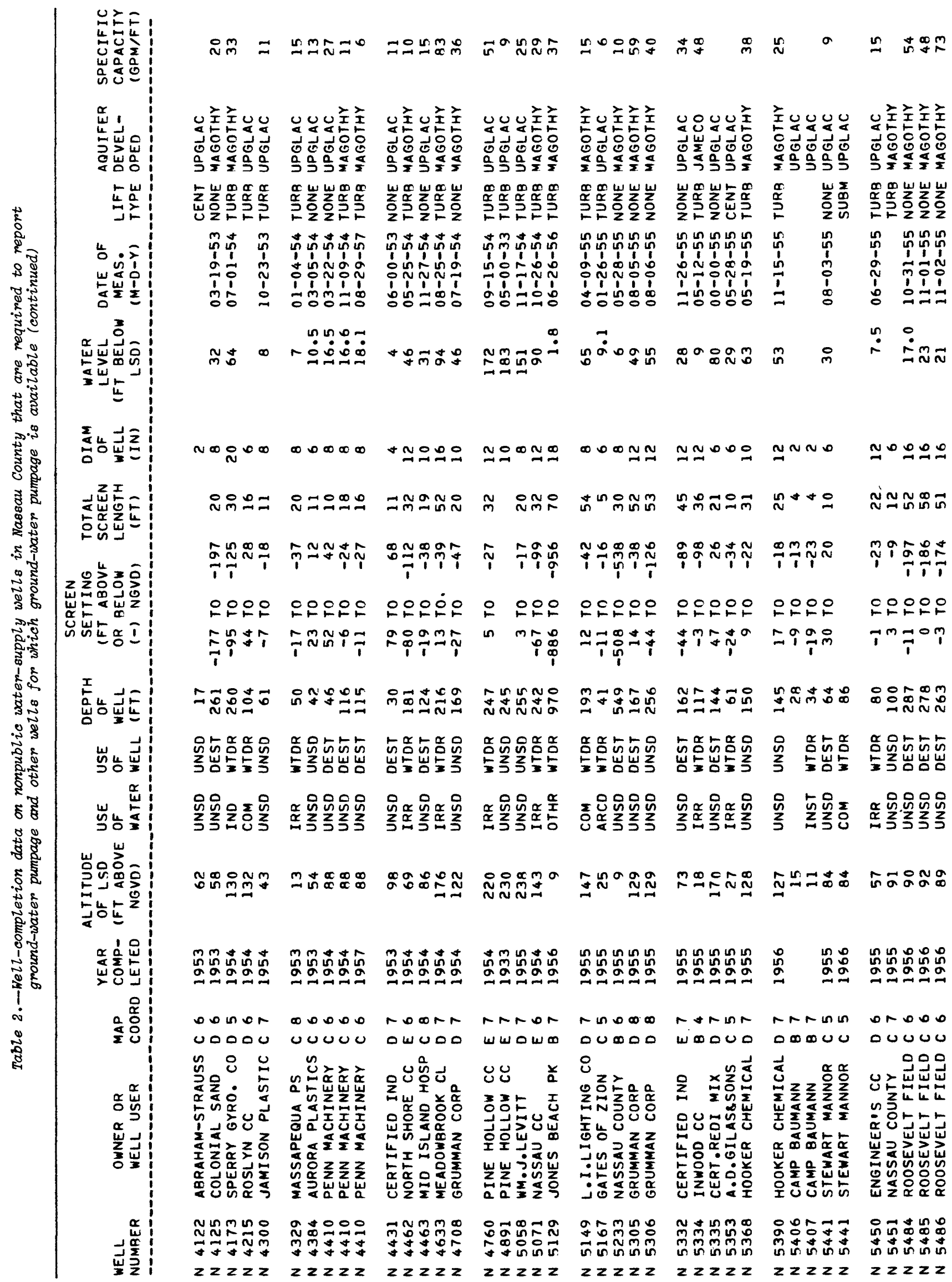




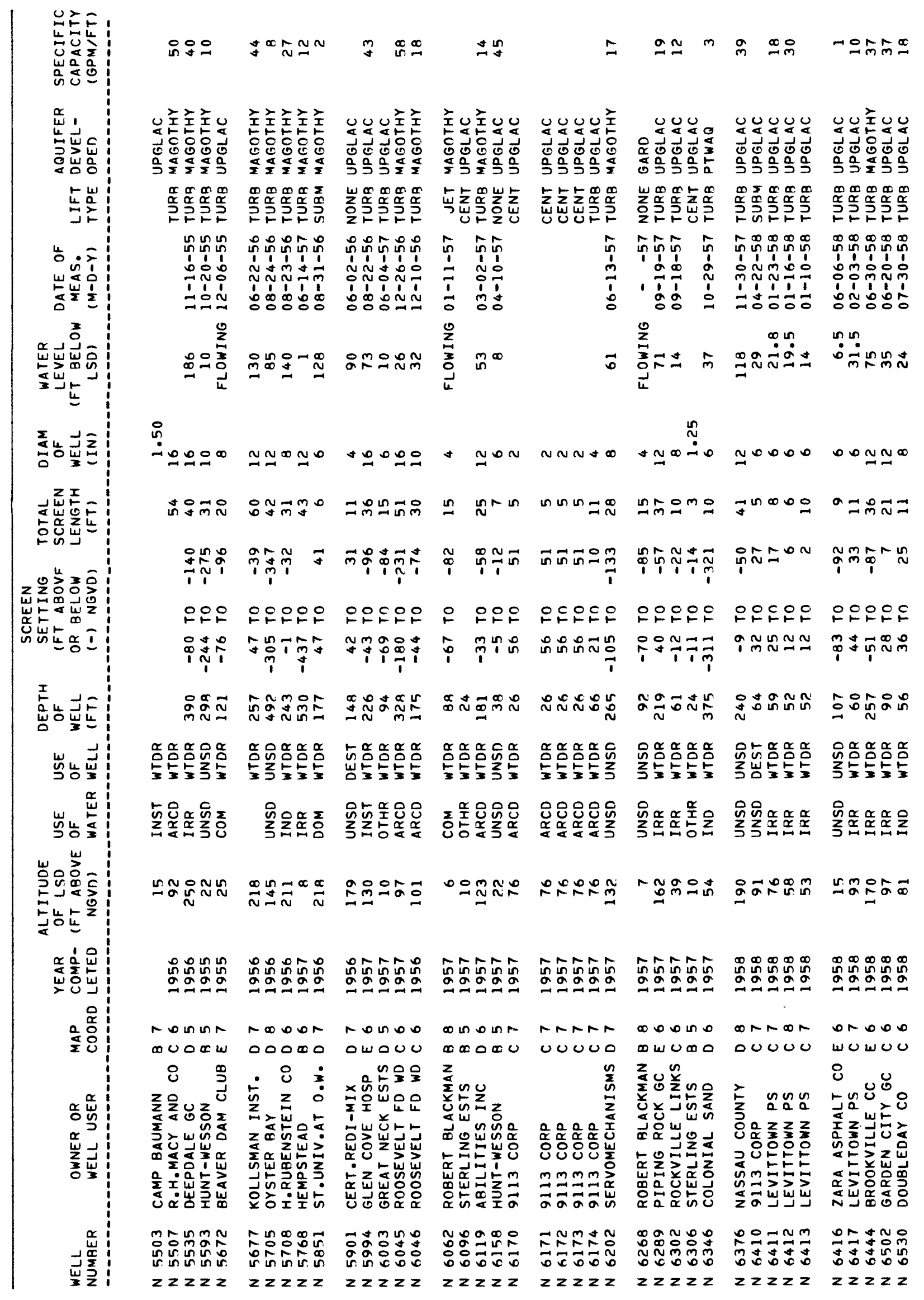




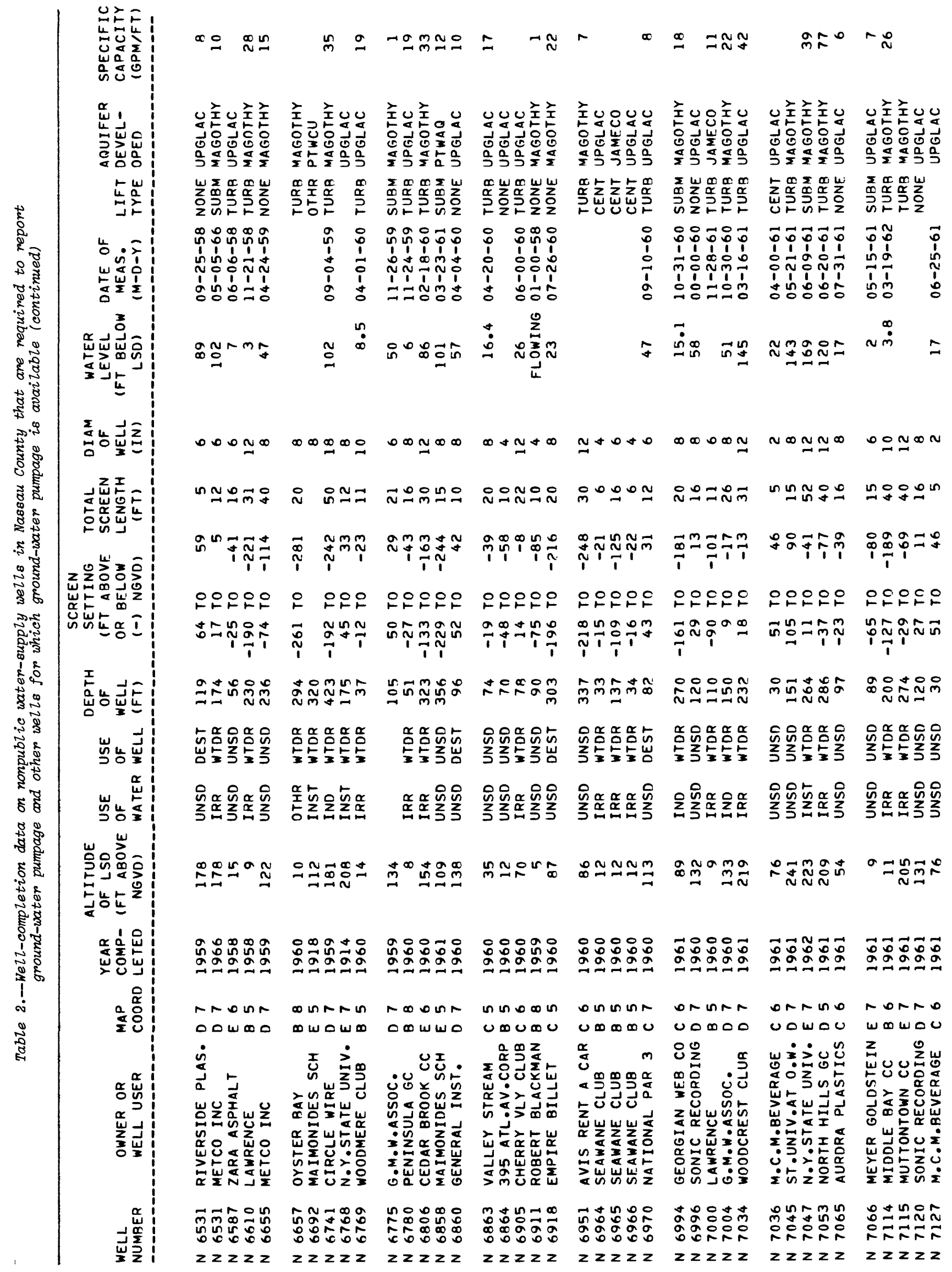




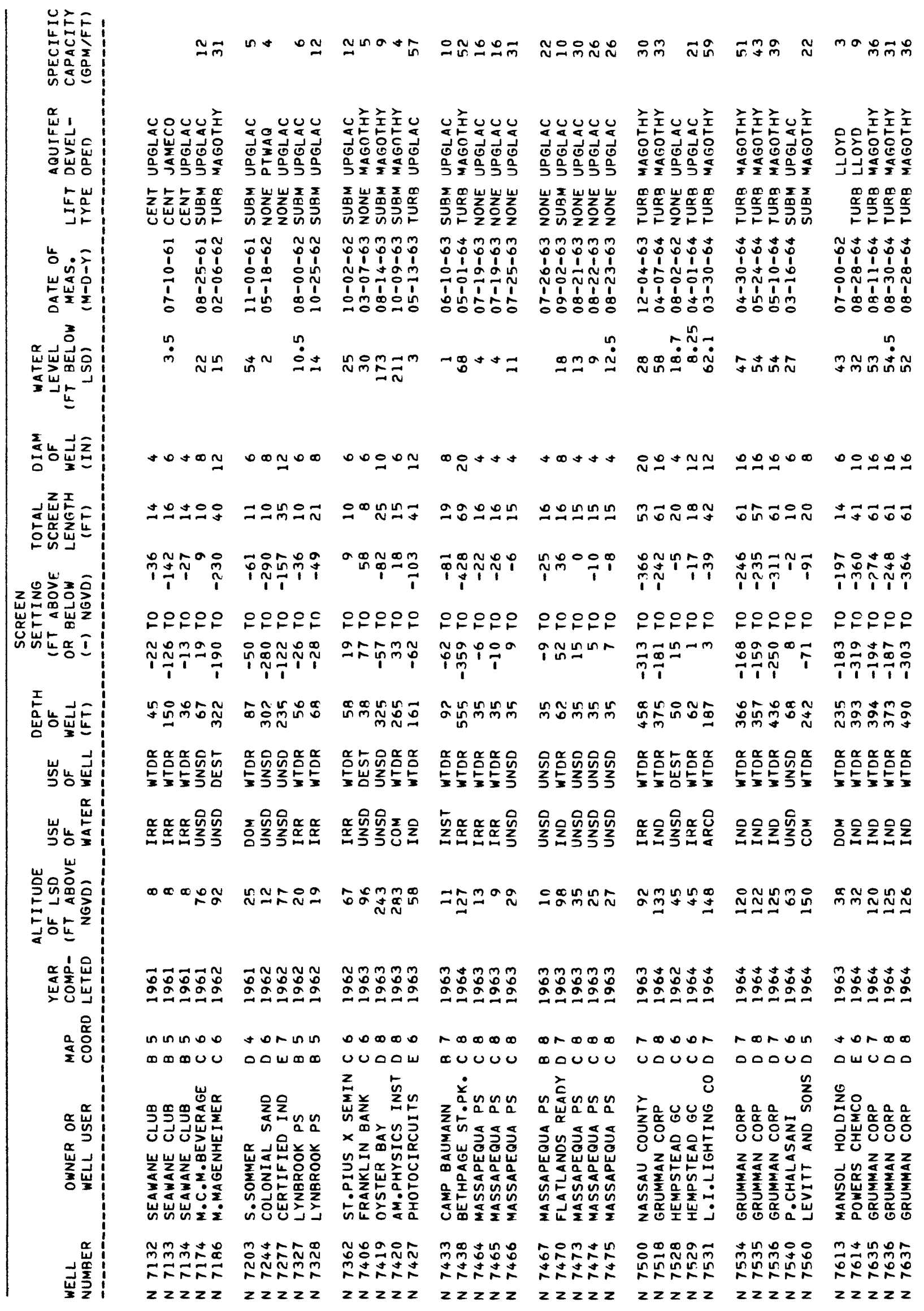




\begin{tabular}{|c|c|c|c|c|c|c|c|c|}
\hline 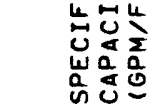 & $\tilde{\sim}^{0} \approx+$ & $m \tilde{n}$ & $\stackrel{\infty}{\infty}$ & $\sigma \stackrel{n}{\sim} m$ & $80 \mathrm{ma}$ & 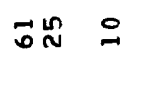 & $\cong \stackrel{n}{\sim} \cong$ & Nิ \\
\hline 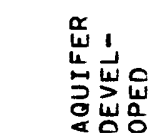 & 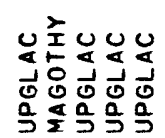 & 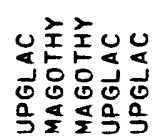 & 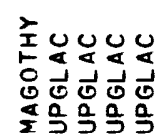 & 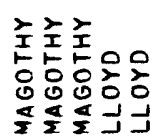 & 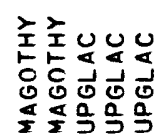 & 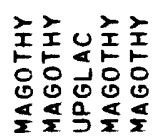 & 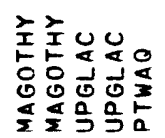 & 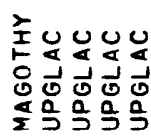 \\
\hline & 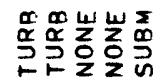 & 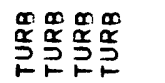 & 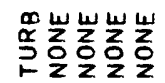 & 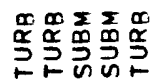 & 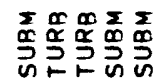 & 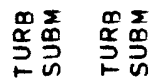 & 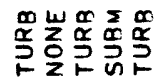 & 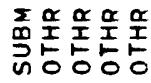 \\
\hline & 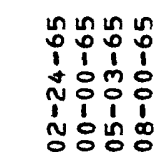 & 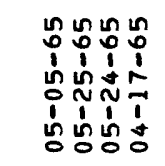 & 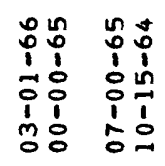 & 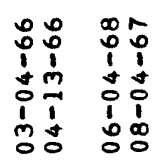 & 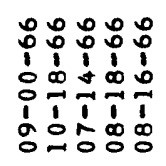 & 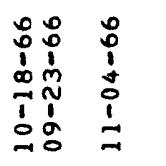 & 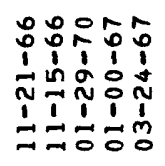 & 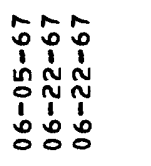 \\
\hline & $\stackrel{n}{0} \operatorname{con}^{-}$ & 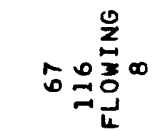 & $\stackrel{\square}{=} \cong \stackrel{n}{\circ}$ & $\dot{n}_{\tilde{D}} \stackrel{\sim}{N}$ & $\underset{\sim}{n} \mp$ × & $i^{N}$ & 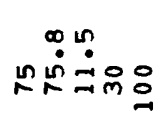 & inn \\
\hline & $\simeq 0+\infty \infty$ & $\simeq \cong \simeq \infty \sim$ & $\stackrel{+}{2}+\infty$ & 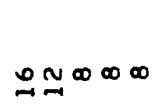 & $0 \underset{0 \infty \infty}{0}$ & $\stackrel{\sim}{\sim} \cong \infty$ & $\simeq 0 \cong 0 \cong$ & 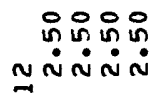 \\
\hline & $\tilde{\sim} \stackrel{\sim}{\sim} \stackrel{0}{\sim} \cong$ & $\vec{N} \vec{m} \vec{m} \hat{N}^{+}$ & 品묘 & $\stackrel{\sim}{\sim} \stackrel{\infty}{N} \Xi \Xi$ & ค: & กี & 욜 & 00000 \\
\hline & พั0 & 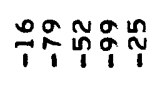 & ํㅜㄴ & 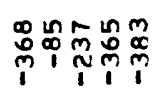 & 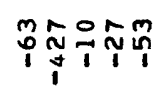 & 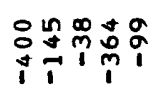 & 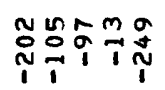 & 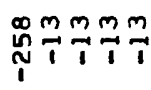 \\
\hline & 안아융ㅇㄴ & 안암요 & 앙ㅇㄴ안 & 안앙ㅇㄴ안 & 앙ㅇㄴㅇㅛ & 은안요 & 안아운요 & 안ㅇㄴ요아 \\
\hline & 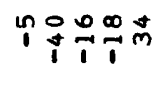 & ก๊ & $\tilde{i}_{1}^{+} \overbrace{i}^{0}$ & 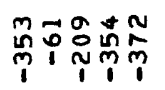 & นำ & 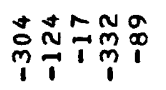 & ํํㄴ & $\underset{\sim}{\mathbb{N}} \uparrow \uparrow \uparrow \uparrow$ \\
\hline & 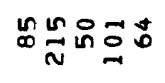 & 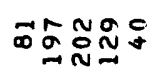 & 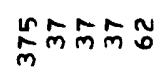 & 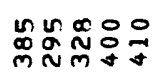 & 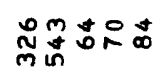 & 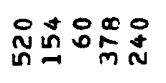 & 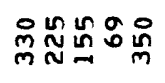 & $\vec{D} \mathbb{N} N \mathbb{N} N$ \\
\hline & 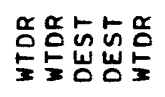 & 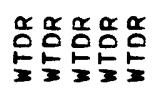 & 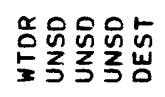 & 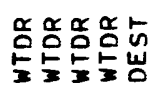 & 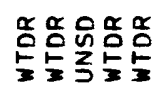 & 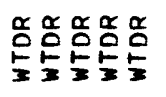 & 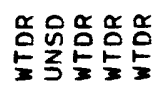 & 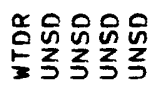 \\
\hline & 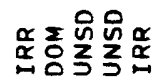 & 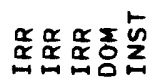 & 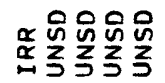 & 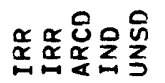 & 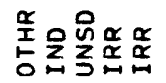 & 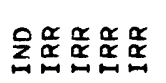 & 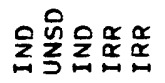 & 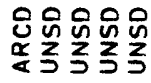 \\
\hline 总 & 苞 & 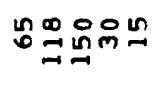 & $\stackrel{\infty}{N} \approx \approx \cong ニ$ & 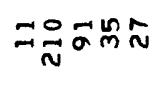 & 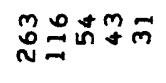 & $\stackrel{D^{\circ}}{N} \underset{N}{ }$ & 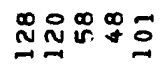 & $\stackrel{m}{0} 0000$ \\
\hline & 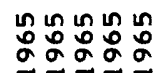 & 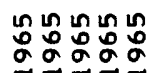 & 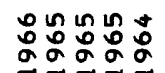 & :용ㅇㅇㅇ & : & : & ㅇํㅇㅇㅇㅇㅇㅇ & โㅇㅇㅇㅇㅇㅇ \\
\hline & 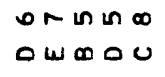 & 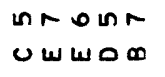 & $\begin{array}{l}-\infty 000 \\
0 \cup 000\end{array}$ & 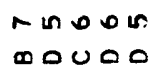 & No & 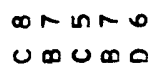 & 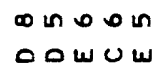 & 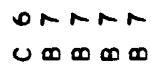 \\
\hline$\underline{\mathbf{w}}$ & 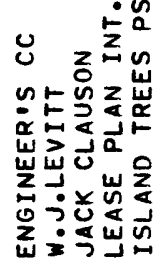 & 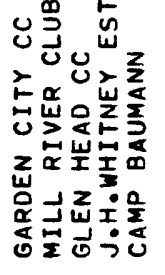 & 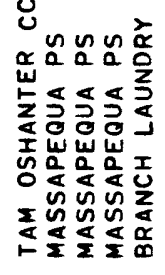 & 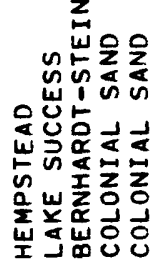 & 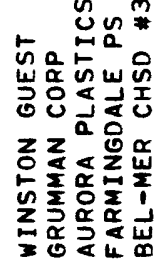 & 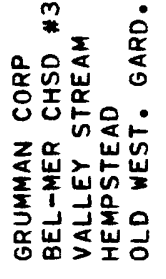 & 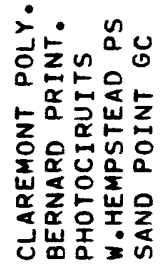 & 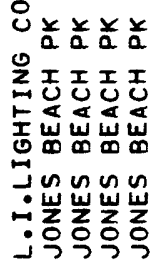 \\
\hline & 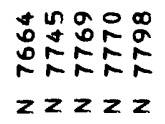 & 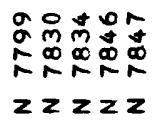 & 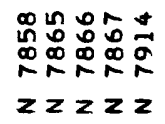 & $\begin{array}{l}\infty \\
0 \\
0 \\
0 \\
0\end{array}$ & 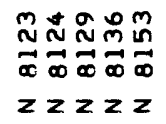 & 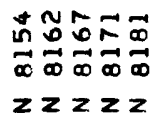 & 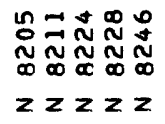 & 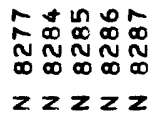 \\
\hline
\end{tabular}




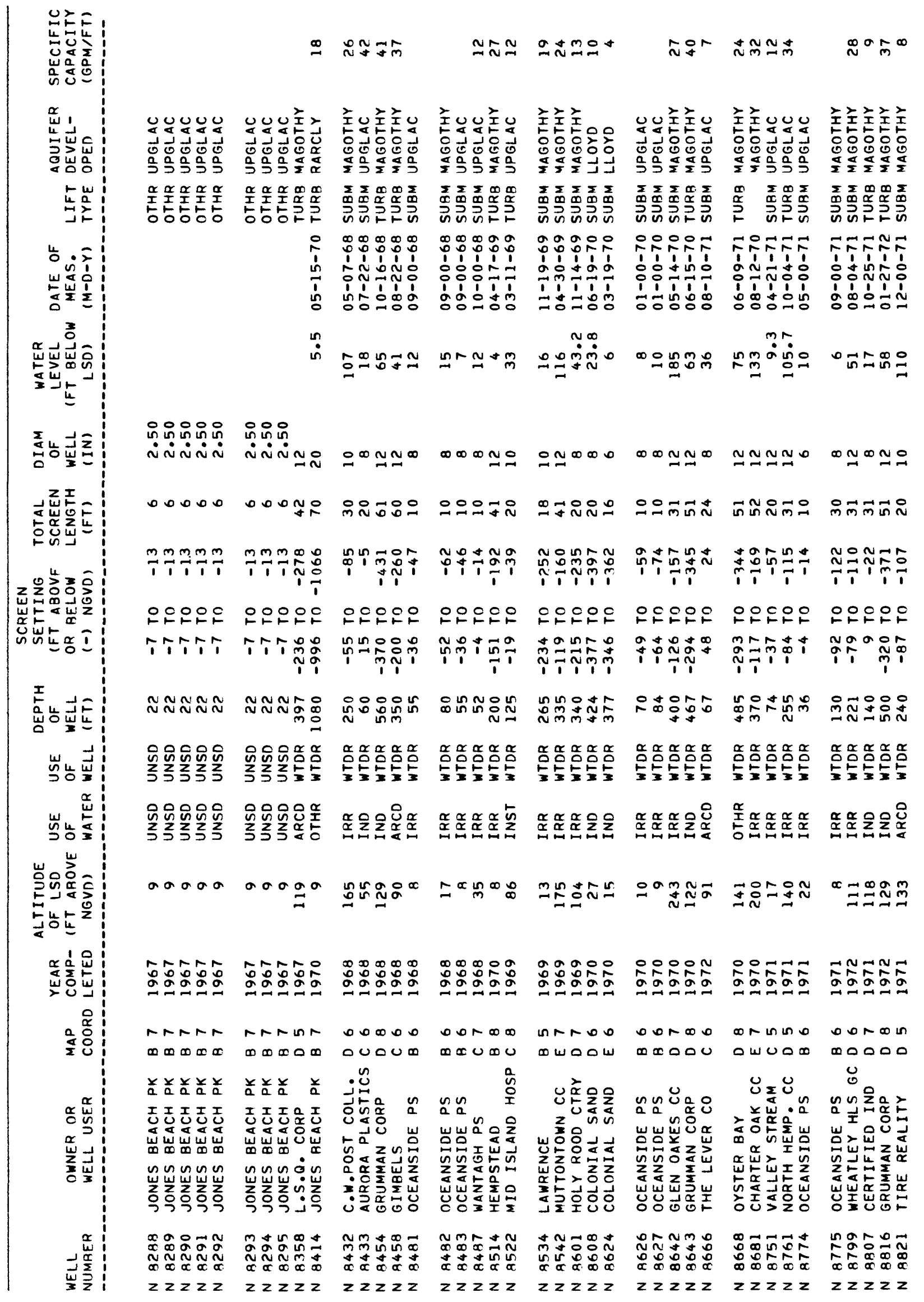




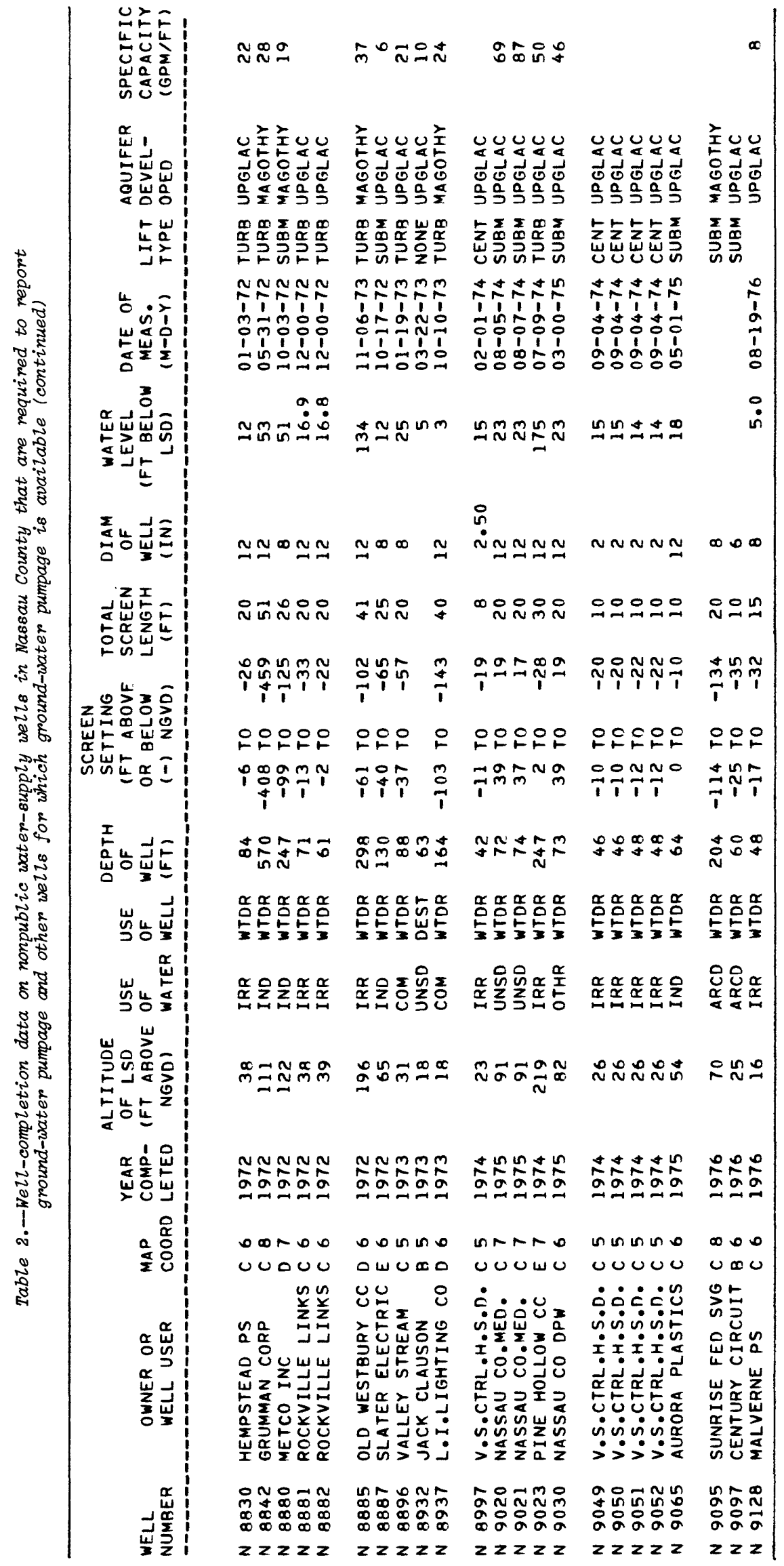

